University of Louisville

ThinkIR: The University of Louisville's Institutional Repository

Electronic Theses and Dissertations

$5-2020$

\title{
Mechanistic insight of the role of NHERF1 in cisplatin-induced acute kidney injury.
}

Adrienne M. Bushau-Sprinkle

University of Louisville

Follow this and additional works at: https://ir.library.louisville.edu/etd

Part of the Medicine and Health Sciences Commons

\section{Recommended Citation}

Bushau-Sprinkle, Adrienne M., "Mechanistic insight of the role of NHERF1 in cisplatin-induced acute kidney injury." (2020). Electronic Theses and Dissertations. Paper 3407.

https://doi.org/10.18297/etd/3407

This Doctoral Dissertation is brought to you for free and open access by ThinkIR: The University of Louisville's Institutional Repository. It has been accepted for inclusion in Electronic Theses and Dissertations by an authorized administrator of ThinkIR: The University of Louisville's Institutional Repository. This title appears here courtesy of the author, who has retained all other copyrights. For more information, please contact thinkir@louisville.edu. 


\title{
MECHANISTIC INSIGHT OF THE ROLE OF NHERF1 IN CISPLATIN-INDUCED ACUTE KIDNEY INJURY
}

By

\author{
Adrienne M. Bushau-Sprinkle \\ B.S. University of Louisville, 2015 \\ M.S. University of Louisville, 2018 \\ A Dissertation \\ Submitted to the Faculty of the \\ School of Medicine of the University of Louisville \\ In Partial Fulfillment of the Requirements \\ for the Degree of \\ Doctor of Philosophy in Pharmacology and Toxicology \\ Department of Pharmacology and Toxicology \\ University of Louisville \\ Louisville, KY
}

May 2020 



\title{
MECHANISTIC INSIGHT OF THE ROLE OF NHERF1 IN CISPLATIN-INDUCED ACUTE KIDNEY INJURY
}

By Adrienne M. Bushau-Sprinkle

B.S. University of Louisville, 2015

M.S. University of Louisville, 2018

Dissertation Approved on

$01 / 22 / 2020$

By the following Committee:

\author{
Eleanor D. Lederer, M.D. (Mentor) \\ Leah J. Siskind, Ph.D. \\ Michelle T. Barati, Ph.D. \\ Michael E. Brier, Ph.D. \\ Michael M. Merchant, Ph.D.
}




\section{DEDICATION}

This dissertation is lovingly dedicated

to my parents

Lawrence and Travis Bushau

and to my husband

Neil Bushau-Sprinkle

for their constant love, encouragement and support

in all my endeavors. 


\section{ACKNOWLEDGEMENTS}

First, I would like to thank my mentor, Dr. Eleanor Lederer, for her steadfast guidance and support in my research and learning. Her encouragement, patience and confidence in my abilities during my first scientific investigations and throughout my graduate career has been instrumental in the success of my research. I would also like to thank my dissertation committee members, Dr. Michelle Barati, Dr. Leah Siskind, Dr. Michael Merchant, and Dr. Michael Brier, for their guidance and support throughout my graduate career.

Thank you Dr. Walter (Burt) Watson for his help collecting and processing samples along with analyzing the HPLC data. Much thanks to Dr. Susan Coventry and Dr. Jessica Hata for help with the electron microscopy and mitochondrial morphology analysis. I would also like to thank Dr. Bradford Hill for his help and resources involved in isolating mitochondria and in evaluating mitochondrial oxygen consumption rate by XF24 Seahorse. Thank yous also go out to Dr. Pawel Lorkiewicz and Dr. David Hoetker for their LC-MS support.

Many thanks to my lab mates who have been an instrumental part of my graduate experience: Susan Isaacs, for her expertise in histology and immunoblot, Dr. Kenneth Gagnon, Dr. Amanda Sherwood, Caryl Conklin, and Kathleen Kitterman for their guidance and lab management. 
Thank you to everyone in the University of Louisville Department of Pharmacology and Toxicology and Division of Nephrology for their support. Thanks also to my cohort mates for making coursework more enjoyable.

Finally, I would also like to give many thanks to my friends and family for their support through my years in graduate school. Special thanks to Christine Taylor and Dakota Briscoe for their steadfast friendship, to my husband, Neil Bushau-Sprinkle, for his endless love, patience and encouragement, and to my parents- Lawrence and Travis Bushau- and extended family for all of their loving support throughout my life that made me who I am today. I am so grateful for each of these individuals, and for the opportunity to do the work that is summarized herein. 


\begin{abstract}
MECHANISTIC INSIGHT OF THE ROLE OF NHERF1 IN CISPLATIN-INDUCED ACUTE KIDNEY INJURY

Adrienne M. Bushau-Sprinkle

January 22,2020
\end{abstract}

Background. Acute kidney injury (AKI) develops in $30 \%$ of patients who receive cisplatin, a widely used chemotherapeutic agent. We previously showed that NHERF1 loss resulted in increased susceptibility to cisplatin nephrotoxicity. The overarching goal of this dissertation was to elucidate mechanisms of susceptibility to cisplatin-induced AKI, specifically the effects of NHERF1 loss on tubule cell metabolism, tubule cell mitochondrial function, and alterations in oxidative state and/or renal handling of cisplatin. Methods. 2-4 month male wild type (WT) and NHERF1 knock out (KO) mice were treated with either vehicle control or cisplatin (20 mg/kg dose IP) for 4,24 , and 72 hours. Urine was collected for NGAL and kidneys were harvested for histology and the following assays: Thiobarbituric Acid Reactive Substances (TBARS) for lipid peroxidation, Y-glutamyl transferase (GGT) activity, and Western Blot for GGT and cysteine Sconjugate beta lyase (CCBL). Mitochondrial respiration was conducted via the Seahorse XF24 analyzer on non-treated isolated kidney mitochondria. LC-MS 
analysis was used to evaluate ATP content in non-treated kidneys. Electron microscopy was utilized to evaluate mitochondrial morphology and number in non-treated kidneys. HPLC of the reduced and oxidized forms of the small molecular weight thiols (glutathione (GSH), glutathione disulfide (GSSG), cysteine (Cys), and cystine (CySS), and cysteine-glutathione disulfide (CySSG) on plasma and kidney cortex. Statistical analysis was completed using Student's t-test for LC-MS, mitochondrial number, and mitochondrial respiration and Twoway ANOVA was used for all other analysis. $P$-values of $<0.05$ were considered statistically significant. Results. Chapter III demonstrates that WT and NHERF1 KO mice do not exhibit metabolic changes or changes in ATP content that would definitively sensitize the KO mice to cisplatin injury. Chapter IV shows that NHERF1 loss does not affect mitochondrial morphology or mitochondrial number, or oxidative phosphorylation capacity via Seahorse XF24 analysis. Thus, mitochondrial dysfunction does not appear to sensitize the KO mice to cisplatin injury. Lastly, Chapter V reveals that NHERF1 KO mouse kidneys do not exhibit changes in lipid peroxidation, oxidative stress, GGT or CCBL protein levels that would sensitize these animals to cisplatin. However, NHERF1 KO kidneys appear to respond differently to the cisplatin insult itself, characterized by differences in GGT activity in response to cisplatin. Conclusions. In conclusion, the work presented in this dissertation reveals that metabolic stress and mitochondrial dysfunction are not the mechanisms of susceptibility to cisplatin in NHERF1 KO mice. Furthermore, NHERF1 loss does not lead to changes in kidney GSH metabolism. In conclusion, these data do not support NHERF1 loss 
resulting in a fundamental metabolic defect that increases susceptibility to cisplatin injury. Instead NHERF1 loss appears to influence either the handling, the initial insult, or the response to injury resulting in exacerbated injury. 


\section{TABLE OF CONTENTS}

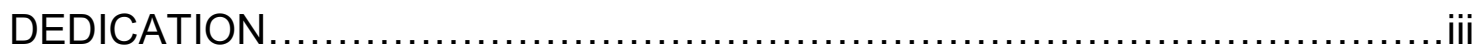

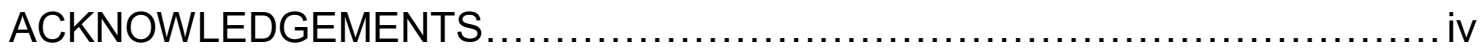

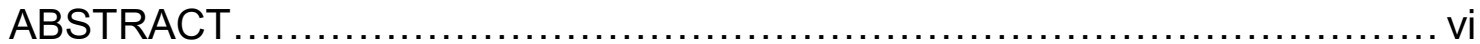

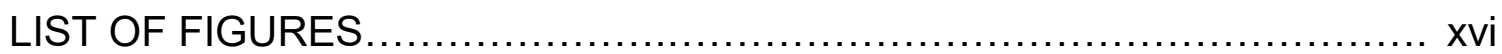

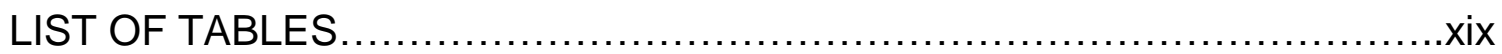

CHAPTER

I. INTRODUCTION

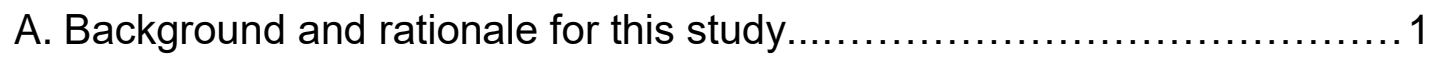

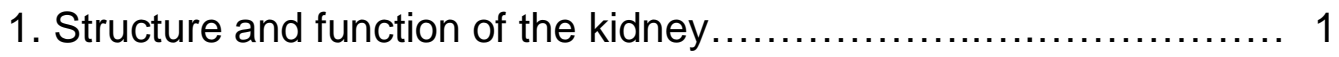

2. Importance of the kidney in human health and disease $\ldots \ldots \ldots \ldots \ldots .4$

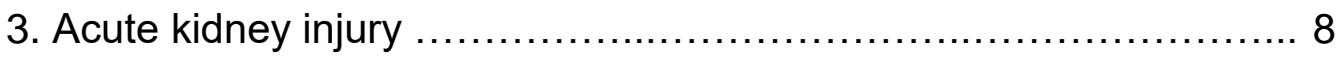

4. History of cisplatin in the clinic.................................... 10

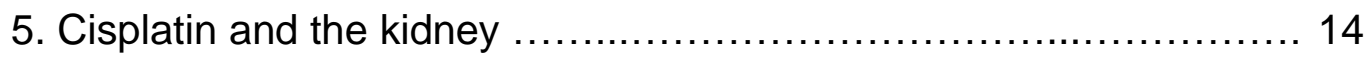

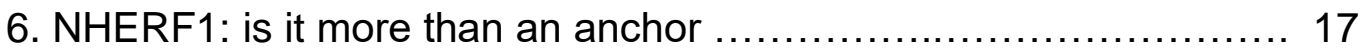

7. Metabolism and susceptibility to cisplatin toxicity ................. 23

8. Mitochondrial dysfunction and susceptibility to cisplatin toxicity .......24

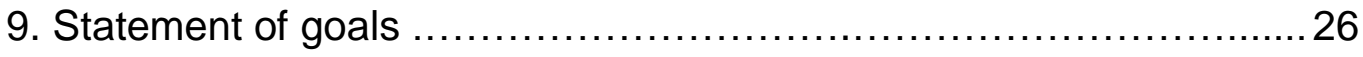

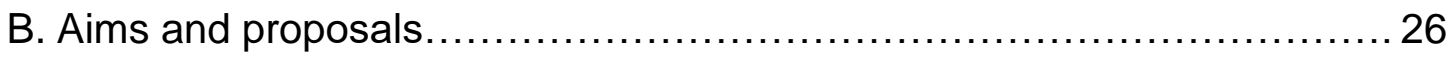

1. Characterization of metabolic differences in kidneys of WT and

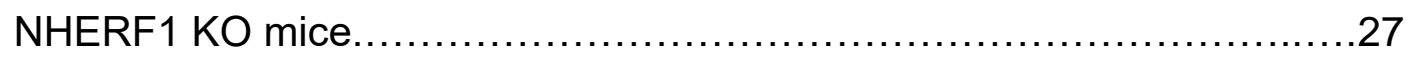

2. Identification of changes in mitochondrial function in NHERF1 KO mice

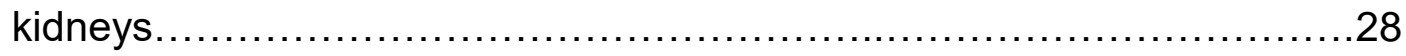


3. Identification of mechanisms for enhanced cisplatin injury in NHERF1

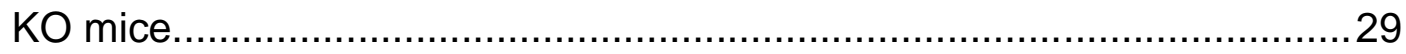

II. EXPERIMENTAL PROCEDURES ..................................... 31

A. Animals and treatments............................................... 31

1. Animal sacrifice, tissue collection, and storage ....................... 32

2. Acute model of cisplatin nephrotoxicity................................32

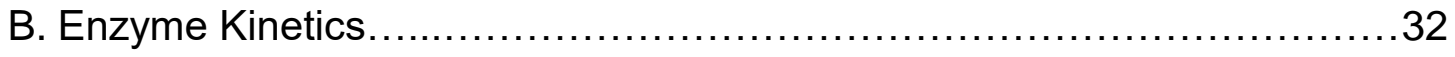

1. Tissue preparation................................................... 32

2. Fructose-1,6-Bisphosphatase activity assay ........................32

3. Glucose-6-Phosphatase activity assay .............................33

4. Lactate Dehydrogenase activity assay ................................34

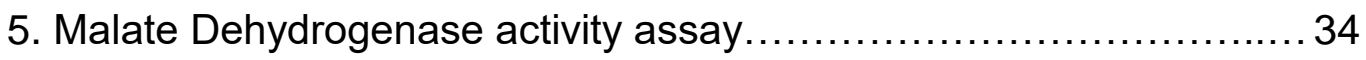

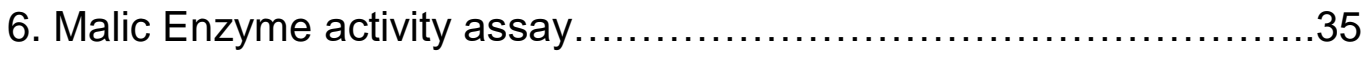

7. Glucose-6-Phosphate Dehydrogenase activity assay .................35

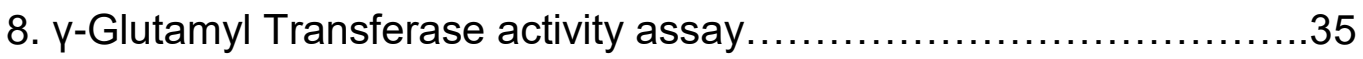

C. Liquid Chromatography-Mass Spectrometry (LC-MS) ......................36

1. LC-MS of kidney cortex for ATP quantification..........................36

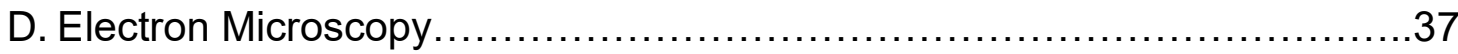

1. Perfusion fixation of total kidney in situ for electron microscopy.........37

E. Seahorse XF24 mitochondrial respiration analysis........................38

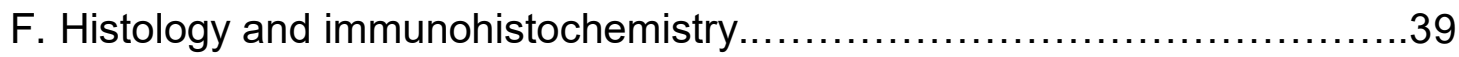

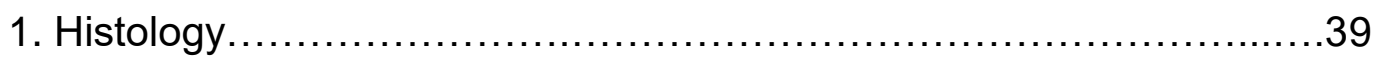

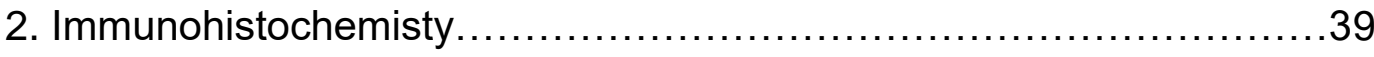




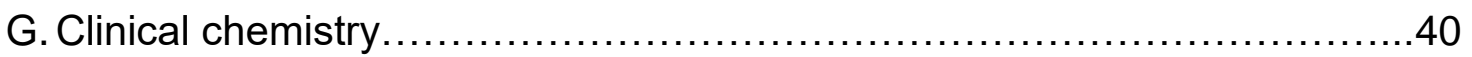

1. Neutrophil gelatinase-associated lipocalin (NGAL) ...................40

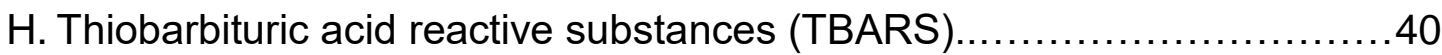

I. High-performance Liquid Chromatography (HPLC) ........................40

1. Collection of mouse blood and tissue for GSH, GSSG, Cys, and

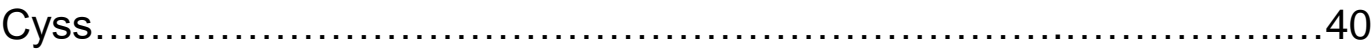

2. HPLC analysis of kidney cortex and plasma for GSH, GSSG, Cys, and

Cyss.

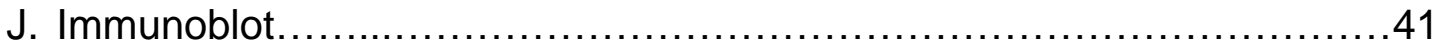

1. Western blot................................................................. 41

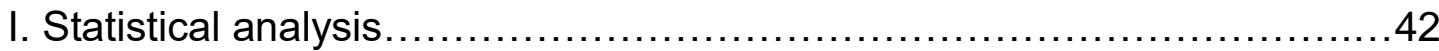

1. Statistical analysis of ATP, mitochondrial number, and respiration

data

2. Statistical analysis of enzyme kinetics, western blots, HPLC, NGAL, and

TBARS

III. NHERF1 LOSS RESULTS IN ALTERED PENTOSE PHOSPHATE

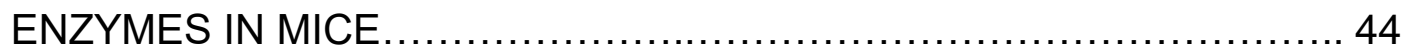

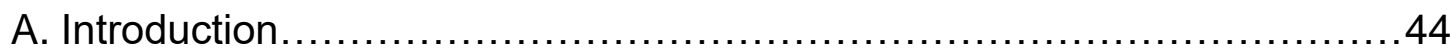

B. Experimental procedures............................................... 46

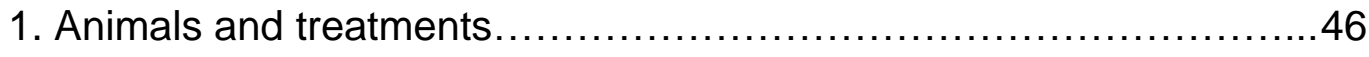

2. Enzyme kinetics...................................................... 46

3. LC-MS of kidney cortex for ATP quantification.........................46

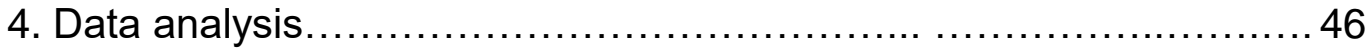


5. Statistical analysis.

C. Results

1. Cisplatin treatment significantly decreases fructose-1,6-bisphosphatase enzyme activity in both WT and NHERF1 KO mice

2. Cisplatin treatment significantly decreases glucose-6-biphosphatase enzyme activity in both WT and NHERF1 KO mice

3. NHERF1 loss or cisplatin treatment did not significantly affect lactate dehydrogenase enzyme activity in mice

4. Cisplatin treatment significantly decreases malate dehydrogenase enzyme activity in both WT and NHERF1 KO mice

5. NHERF1 loss affects malic enzyme activity before and after cisplatin treatment.

6. NHERF1 loss affects glucose-6-phosphate dehydrogenase enzyme activity before and after cisplatin treatment.

7. NHERF1 loss does not affect amount of ATP by LC-MS in mouse kidneys

D. Discussion .57

IV. MECHANISM OF SUSCEPTIBILITY OF NHERF1 KO MICE TO CISPLATIN INDUCED AKI IS NOT MITOCHONDRIAL DYSFUNCTION...............63

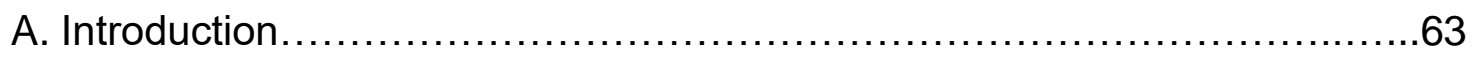

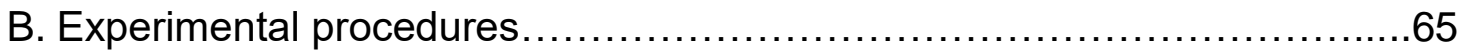

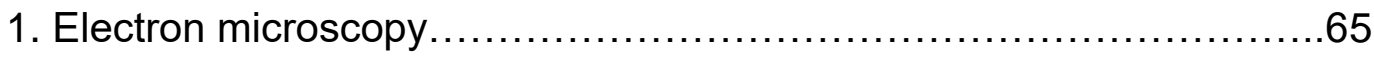

2. Seahorse XF24 mitochondrial respirational analysis...................66 


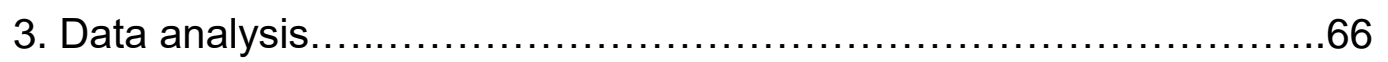

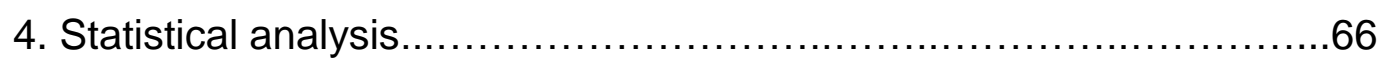

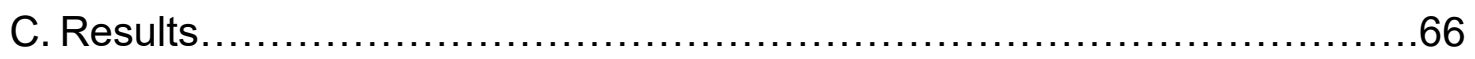

1. NHERF1 loss does not affect kidney proximal tubule mitochondria

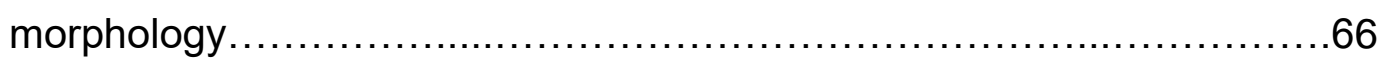

2. NHERF1 loss does not affect mitochondria number or area in kidney

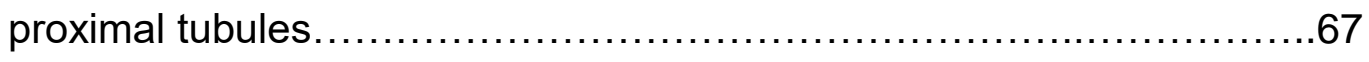

3. WT and NHERF1 KO mouse kidney mitochondria have similar oxidative

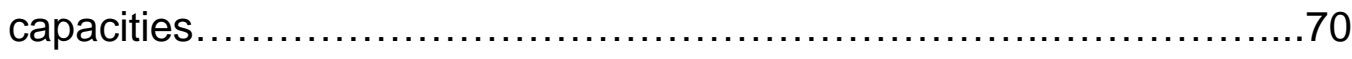

D. Discussion..................................................................... 73

V. NHERF1 LOSS AFFECTS GGT ACTIVITY IN RESPONSE TO

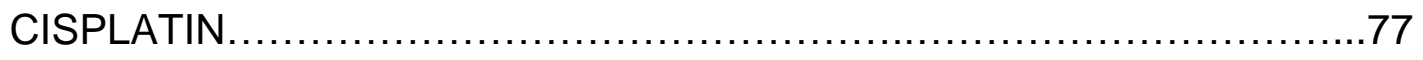

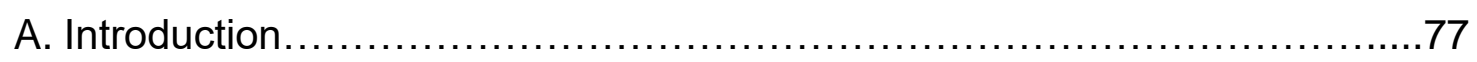

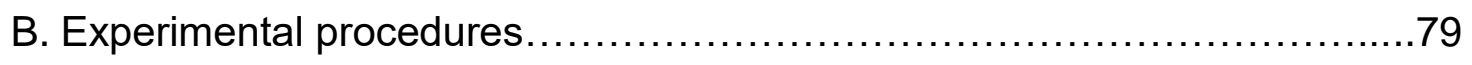

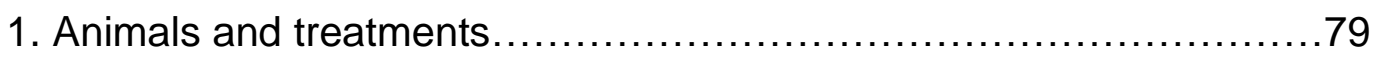

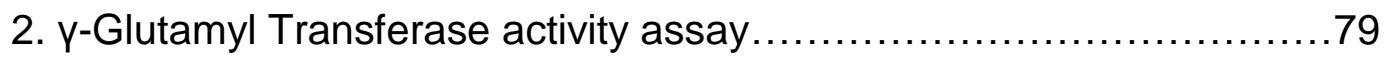

3. Histology and immunohistochemistry ................................ 80

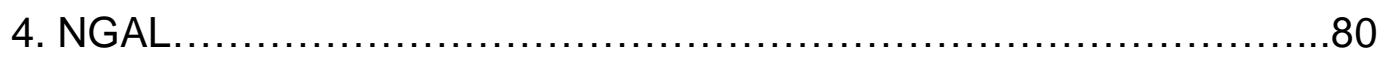

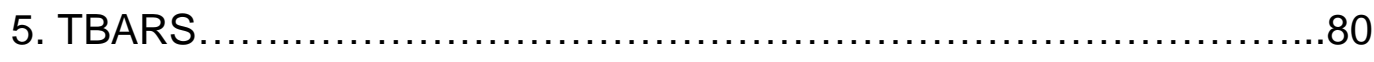

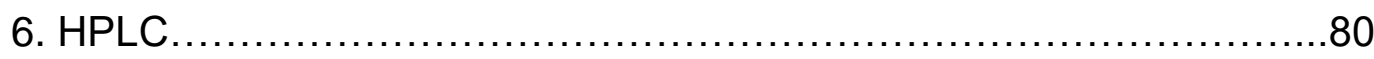

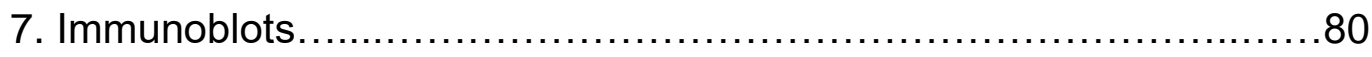

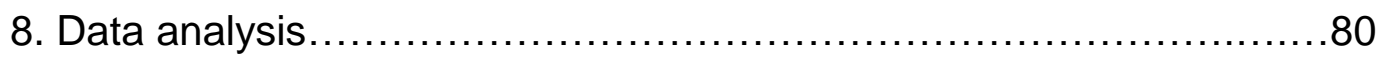

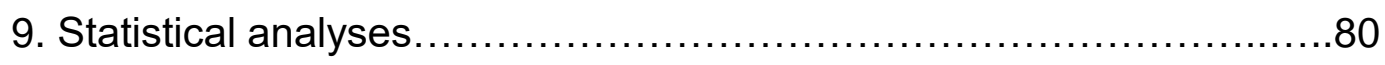


C. Results.

1. NHERF1 loss results in early cisplatin-induced renal histologic injury.

2. Cisplatin treatment significantly increases urine NGAL protein in NHERF1 KO mice in comparison to cisplatin treated WT at 24 hours

3. NHERF1 loss does not affect lipid peroxidation by TBARS.

4. NHERF1 loss increases 4-hydroxynonenal (4-HNE) in cortex and juxtamedullary region following cisplatin treatment. .84

5. NHERF1 KO kidneys do not have altered GSH metabolism.

6. NHERF1 loss affects GGT activity in response to cisplatin insult......92

7. NHERF1 loss does not affect GGT protein.

8. GGT localization is altered in NHERF1 KO kidneys 24 hours after cisplatin treatment. .96

9. NHERF1 loss does not affect CCBL protein. 96

D. Discussion 98

VI. DISCUSSION AND CONCLUSIONS ................................. 105

A. Restatement of goals and questions .................................. 105

B. Major findings of this dissertation ...................................... 105

1. NHERF1 loss results in altered pentose phosphate enzymes in mice. 105

2. Mechanism of susceptibility of NHERF1 KO mice to cisplatin-induced AKI is not mitochondria dysfunction. 107 
3. NHERF1 loss affects GGT activity in response to cisplatin

C. Significance of new findings.

D. Strengths and weaknesses of this dissertation

1. Strengths

2. Weaknesses

E. Future directions

1. Is the increased ME and G6PD activity identified in Chapter III a compensatory mechanism to maintain the cellular redox state in NHERF1 KO mice?

2. Do NHERF1 KO mitochondria function normally in whole tissue or with changes in substrates? 122

3. Does cisplatin damage the NHERF1 KO mitochondria to a greater degree than WT?

4. Does NHERF1 loss result in altered renal handling of cisplatin besides GGT activity?

5. How do NHERF1 KO mice respond to a chronic model of cisplatin nephrotoxicity?

6. Do heterozygous NHERF1 KO mice have some protection to cisplatin nephrotoxicity?

7. Does NHERF1 loss predispose mouse kidneys to other nephrotoxins? 125

F. Summary and Conclusions. 125 


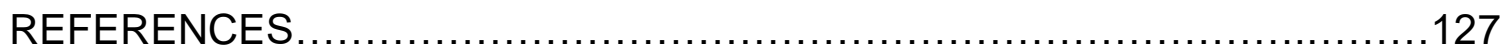

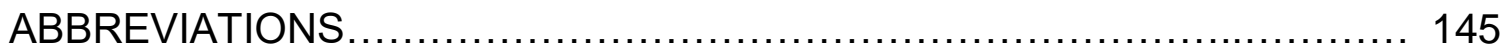

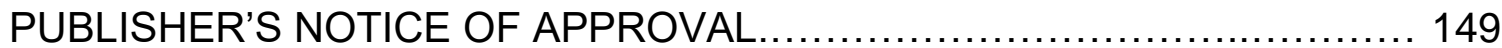

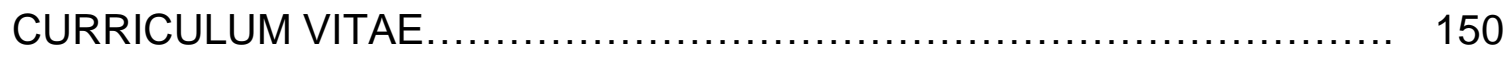




\section{FIGURES}

\section{LIST OF FIGURES}

Figure 1.1: Structure of the mammalian kidney …...................................... 2

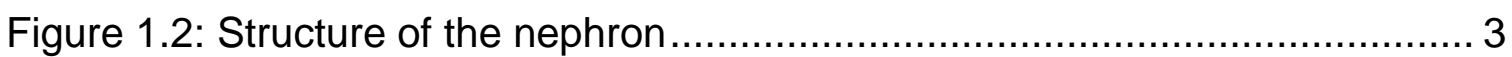

Figure 1.3: Structure of cis-diamminedichloroplatinum (II) or cisplatin .............. 12

Figure 1.4: Cisplatin metabolism to a nephrotoxin..............................16

Figure 3.1: Effect of cisplatin treatment on fructose-1,6-bisphosphatase enzyme

activity in WT and NHERF1 KO mouse kidneys..............................48

Figure 3.2: Effect of cisplatin treatment on glucose-6-phosphatase enzyme activity in WT and NHERF1 KO mouse kidneys ..............................50

Figure 3.3: Lactate dehydrogenase enzyme activity in WT and NHERF1 KO

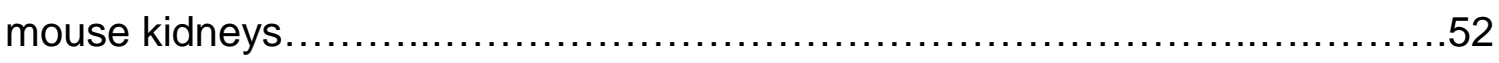

Figure 3.4: Malate dehydrogenase enzyme activity in WT and NHERF1 KO

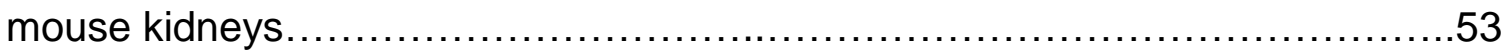

Figure 3.5: Effect of NHERF1 loss and cisplatin treatment on malic enzyme activity in WT and NHERF1 KO mouse kidneys.................................55

Figure 3.6: Effect of NHERF1 loss and cisplatin treatment on glucose-6phosphate dehydrogenase enzyme activity in WT and NHERF1 KO mouse

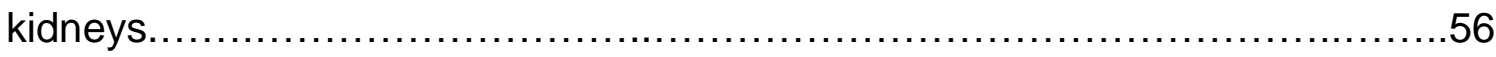

Figure 3.7: ATP content of WT and NHEF1 KO mouse kidneys ...................... 58 Figure 4.1: Electron microscopy of WT and NHERF1 KO proximal tubule

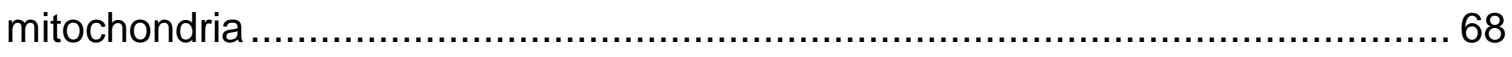


Figure 4.2: Mitochondrial number and area of WT and NHERF1 KO mouse

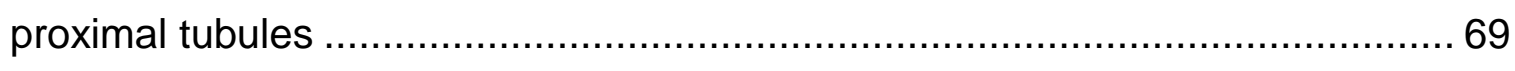

Figure 4.3: Mitochondrial function in isolated mitochondria of WT and NHERF1

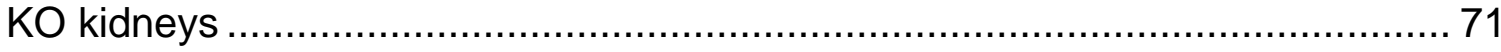

Figure 5.1: Early histologic effect of cisplatin on WT and NHERF1 KO

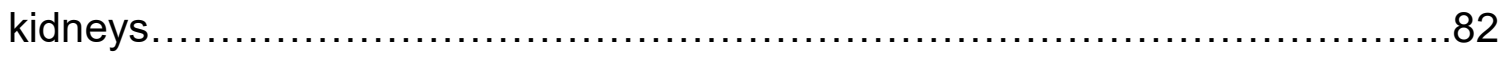

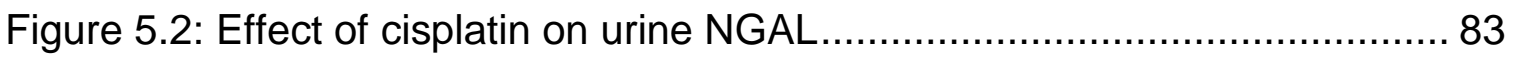

Figure 5.3: Cisplatin's effect on lipid peroxidation by TBARS in WT and NHERF1

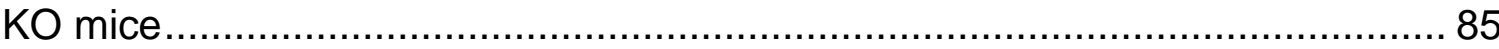

Figure 5.4: The effect of NHERF1 loss and cisplatin treatment on 4-HNE staining

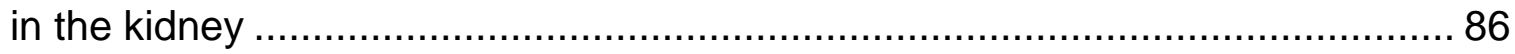

Figure 5.5: Cisplatin and NHERF1 loss effect on small molecular weight thiols. 88 Figure 5.6: Effect of NHERF1 on GGT activity following cisplatin treatment ...... 94 Figure 5.7: Effect of NHERF1 loss on GGT protein .......................................... 95

Figure 5.8: GGT localization in vehicle and cisplatin treated kidneys............97

Figure 5.9: Effect of NHERF1 loss on CCBL protein............................99 


\section{TABLES}

\section{LIST OF TABLES}

TABLE 5.1: Summary of the effect of NHERF1 and cisplatin on small molecular

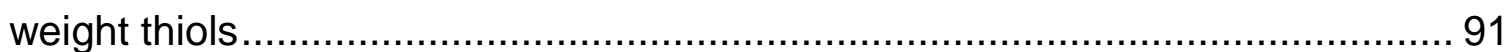




\section{CHAPTER I}

\section{INTRODUCTION}

\section{A. Background and rationale for this study}

\section{Structure and function of the kidney}

In humans and in most mammals the normal kidney is bean shaped and found in pairs within the abdominal cavity behind the peritoneum $(1,2)$. Each kidney is covered by a fibrous capsule with a slit located in the central concave portion of the kidney called the hilum $(1,2)$ (Figure 1.1). The hilum serves as both the entry site for the renal artery and the exit site of the renal vein $(1,2)$. The hilum opens into the renal sinus, composed of shallow urine-filled spaces $(1,2)$. Once bisected the kidney is composed of 3 main regions, the cortex, the outer medulla, and the inner medulla $(1,2)$ (Figure 1.1). The cortex is comprised of glomeruli (clumps of capillaries) and tubules (convoluted epithelial structures), while the medulla lacks glomeruli and consists of parallel arrangements of tubules $(1,2)$.

The nephron is the functional unit of the kidney and it consists of a glomerulus and a tubule (Figure 1.2). The glomerulus is a group of blood vessels that forms filtrate from blood plasma, while the epithelial tubule is designed to 


\section{Figure 1.1}

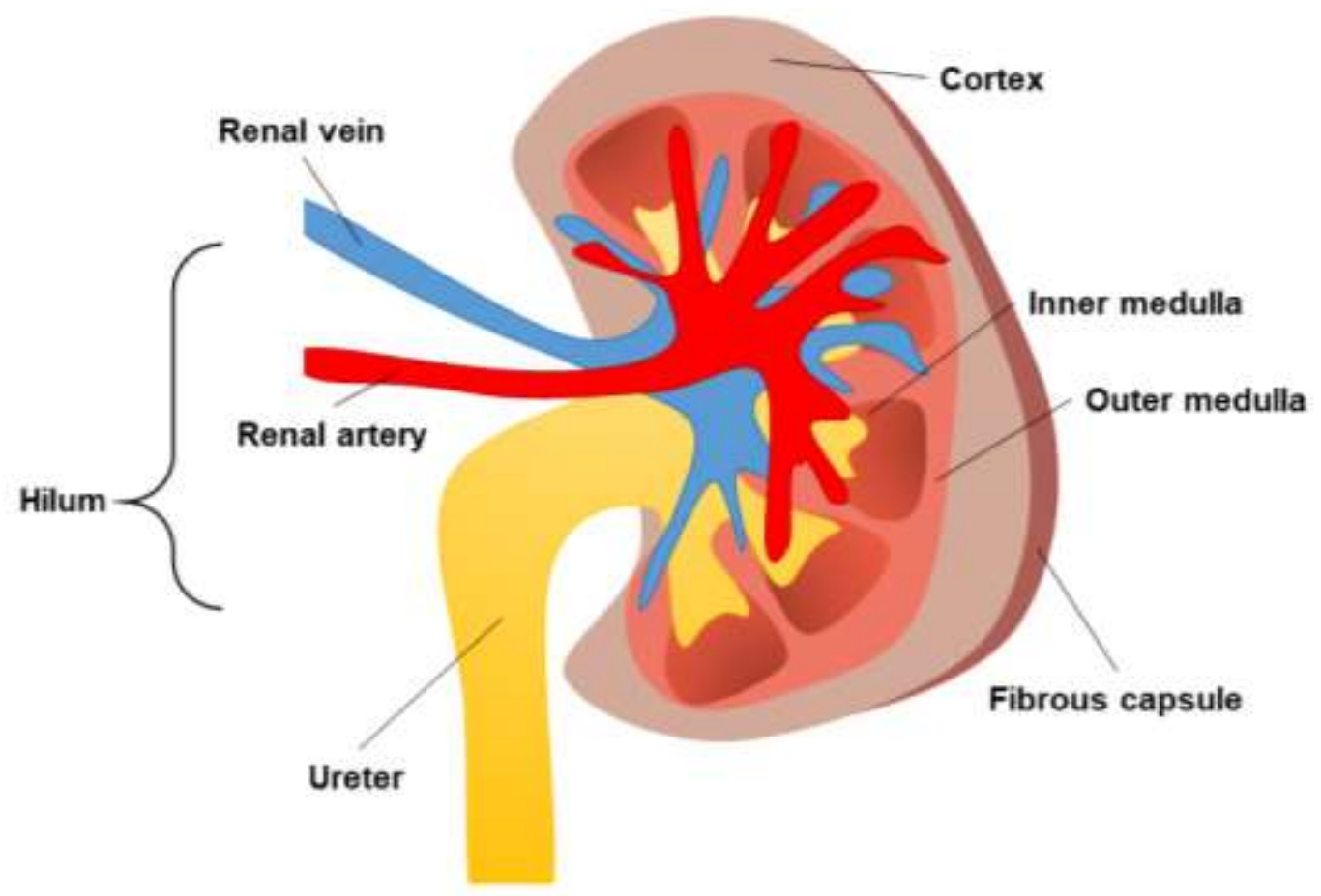

Figure 1.1: Structure of the mammalian kidney.

The kidney structure includes three regions: an outer cortex, outer medulla, and inner medulla. The arteries and veins that support the kidney enter and exit at the hilum. Additionally, the hilum serves as a point of exit for the ureter. 


\section{Figure 1.2}

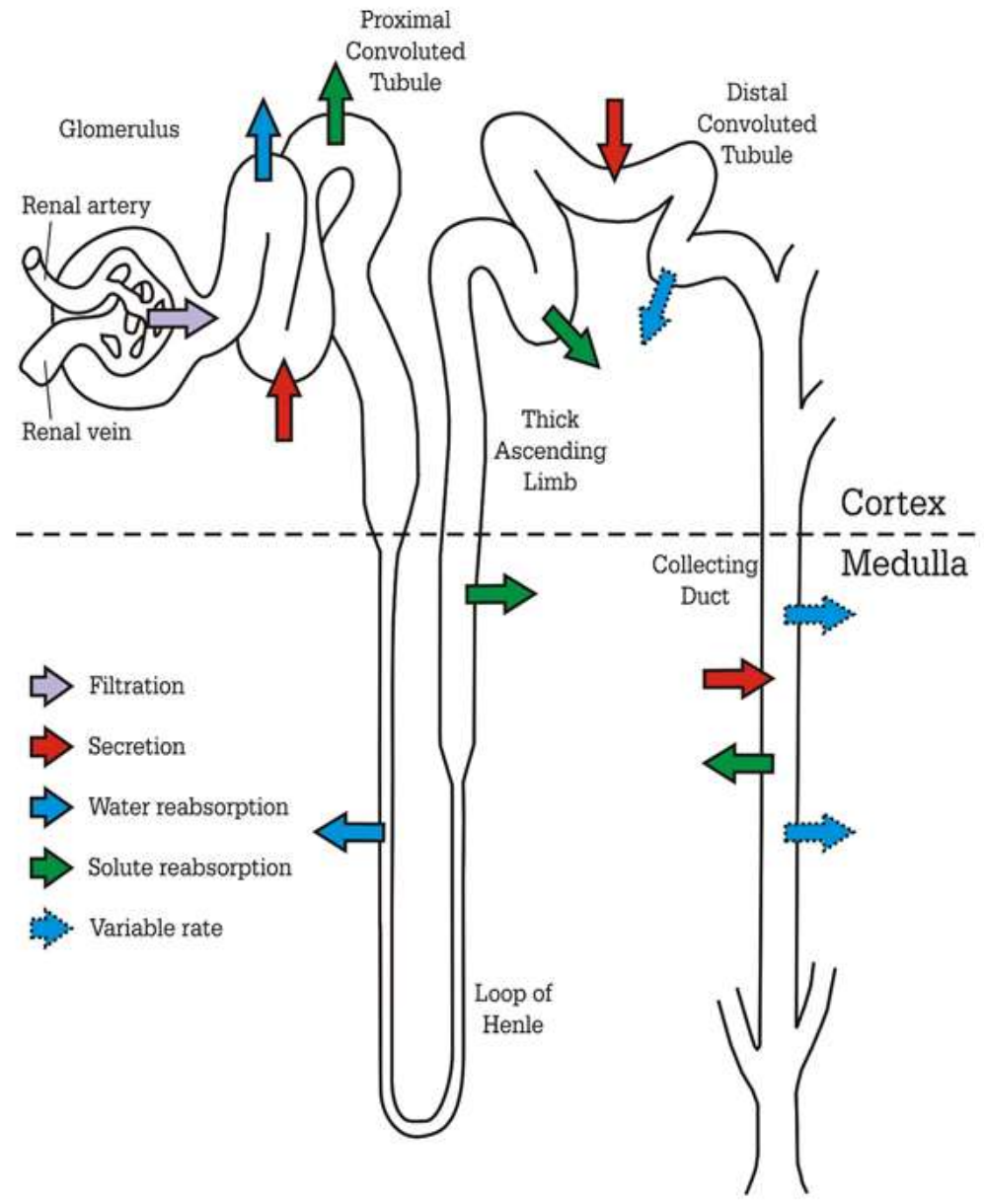

Figure 1.2: Structure of the nephron.

The nephron is the functional unit of the kidney and is responsible for removing wastes. Both the glomerulus and the sections of the tubules of the nephron are in the cortex of the kidney. 
convert the blood plasma filtrate into urine $(1,2)$. Bowman's capsule surrounds the glomerulus and the site where the filtrate passes from the vascular to the tubule system $(1,2)$. The epithelial tubule is divided into multiple subdivisions called the proximal tubule, the thin descending and ascending limb of the loop of Henle, the thick ascending limb of the loop of Henle, the distal convoluted tubule, and the collecting tubule (2) (Figure 1.2). The tubules of the kidney are instrumental in recovery of most of the fluid and solutes filtered from the glomerulus (1).

The proximal tubule recovers the vast majority of filtrate from the glomerulus and is involved in regulating acid-base balance (2), salt and water homeostasis, recovery of filtered glucose and amino acids, as well as divalent ion metabolism (1, 2). Additionally, the proximal tubule is the site for organic anion and cation transport, including drugs. The loop of Henle creates the conditions required for the generation of a concentrated or dilute urine, by pumping salts and urea into the medulla to establish the progressively hypertonic cortical to medullary gradient within the renal interstitium $(1,2)$. The creation of this osmotic gradient enables the regulated movement of water from tubule to interstitium, thus determining urine osmolality. The distal tubule and collecting duct system are sites for more precise control of water and salt excretion via several hormones (e.g., aldosterone, arginine vasopressin) $(1,2)$. All of these structures collectively enable the kidney to form a filtrate of blood plasma and to selectively reabsorb the tubule fluid or secrete solutes into it. 


\section{Importance of the kidney in human health and disease}

The kidneys are multifunctional organs involved in maintaining blood volume, total body salt and water balance, acid-base balance, and bone strength and integrity (1). The kidneys accomplish these tasks through cooperation with other organ systems. Each of these tasks are important in human health, and disruption of one or more of these functions can result in complications and/or the development of disease $(1,3)$.

The human body intakes water, salt, and electrolytes at varying degrees that can interrupt the body's balance of these substances. The kidneys have the ability to vary their excretion of electrolytes and water to maintain this balance (1, 3). Another aspect of this balance of water, salts, and electrolytes is the regulation of plasma osmolality, the combined concentration of these substances $(1,3)$. Osmolality is altered whenever the input and output of water and solutes shift disproportionally $(2,3)$. Thus, the kidneys must excrete water and solutes to match the intakes at rates that maintain the ratio of solutes and water close to a constant value of 292 milliosmoles per kilogram $(\mathrm{mOsm} / \mathrm{kg})(2,3)$. In addition to excreting excess amounts of substances the kidney also responds to shortages. To accomplish this response the kidneys will reduce the output leading to the restoration of this balance of water and solutes $(1,3)$. One of the major actions of the kidney is to regulate all of the electrolytes, body water, and plasma osmolality independently $(2,3)$.

The kidneys work in tandem with other organ systems including the cardiovascular system $(1,2)$. The kidneys regulate systemic blood pressure by: 
[1] determining blood volume which controls cardiac output (heart rate $x$ stroke volume); and [2] producing hormones that regulate vascular resistance (systemic blood pressure/cardiac output) $(2,3)$. It is vital that the kidneys maintain extracellular fluid volume, which includes blood plasma (1-3). This maintenance ensures that there is sufficient volume to fill the vascular space, allowing blood to circulate normally (1-3). Maintenance of the extracellular fluid volume is a result of the kidneys' regulation of water and salt balance $(2,3)$. In addition to maintaining adequate volume of the cardiovascular system, the kidneys produce vasoactive substances (e.g., renin-angiotensin-aldosterone system) that help control vascular smooth muscle $(2,3)$. This influences peripheral vascular resistance and consequently systemic arterial blood pressure (1-3). Disruption of this aspect of renal function leads to the development of hypertension $(1,3)$.

The human body constantly forms the end products of metabolic processes, which must be excreted at the same rate that they are produced $(1,2)$. These products include urea from protein, uric acid from nucleic acids, creatinine from muscle creatinine, urobilin from hemoglobin breakdown, and the metabolites of various hormones and biochemical processes $(2,3)$. Collectively these products are called uremic toxins when they accumulate under conditions of kidney failure (3), and contribute to uremia, the constellation of clinical features characteristic of advanced chronic kidney disease (CKD) $(1,3)$. In addition, the kidneys are responsible for the excretion of many types of drugs. Frequently these drugs damage the kidneys leading to alterations in kidney function. 
Although the kidneys are responsible for excretion of hormones and their metabolites, they are also involved in production of the hormone erythropoietin (1-3). Interstitial cells located in the cortical intersititum (border between renal cortex and medulla) secrete this hormone that stimulates the production of red blood cells in the bone marrow $(1,2)$. The reduction of partial oxygen pressure stimulates the kidney cells to secrete erythropoietin $(2,3)$. In cases of anemia, blood loss, or insufficient blood flow to the kidneys the local oxygenation drops and triggers secretion of erythropoietin (1-3).

The kidneys in combination with the gastrointestinal (Gl) tract are essential regulators for phosphate and calcium homeostasis, which is necessary for bone health (1-3). The kidneys accomplish this homeostasis through the production of calcitriol (active form of vitamin D) (1-3). Calcitriol helps increase calcium absorption from the intestine into the blood. CKD can disrupt this process and cause a form of bone disease, renal osteodystrophy $(1,3)$. Moreover, the impaired bone metabolism is accompanied by calcium-phosphate deposits in blood vessels, increasing the risk of heart disease (3).

Lastly, the kidneys are responsible for maintaining tight control of acid-base balance and they are a site of gluconeogenesis (body's process of producing glucose from non-carbon sources) (1-3). Disruption in acid-base balance can lead to life-threatening conditions such as metabolic acidosis and alkalosis (1-3). Loss of gluconeogenesis can result in hypoglycemia in advanced CKD, particularly under conditions of stress or nutrient deficiency. 
Altogether the kidney is a unique organ that is involved in many vital processes. The kidneys excrete many substances and help maintain the body's homeostasis. Equally important to the kidneys' ability to excrete waste, the kidneys also work in partnership with other organ systems through production of hormones. Consequently, alterations in any of these processes can lead to complications and the development of disease in the body, further illustrating the importance of the normal functioning kidney to overall human health.

\section{Acute kidney injury}

Acute kidney injury (AKI) is defined as an abrupt decline in kidney function, and is a common clinical condition; approximately $20 \%$ of all hospital admissions are related to $\mathrm{AKI}$ (4). 2-300 per million cases of $\mathrm{AKI}$ are severe enough to require dialysis (5). Meanwhile, 2-3000 per million cases of AKI do not require dialysis (5). In the critical care setting close to two thirds of patients will develop $\mathrm{AKI}$ and around five percent of intensive care unit (ICU) patients will develop severe cases of AKI that warrant dialysis (5). AKI is associated with a four-fold increase in mortality and is a risk factor for the development of CKD (5).

AKI can be divided into three pathophysiological categories: pre-renal, intrinsic, and post-renal (obstructive) (2). Pre-renal AKI results from a decrease in arterial blood volume leading to kidney hyperfusion (2), but without intrinsic damage to the kidney tissue. Hemorrhage from trauma or surgery, septic shock, and cirrhosis are all conditions that can lead to the development of pre-renal AKI (2). Intrinsic AKI occurs from direct damage to the kidneys resulting in a sudden 
loss in kidney function. The common causes are acute tubular necrosis (ATN), acute glomerulonephritis (AGN), and acute interstitial nephritis (AIN) (2). Ischemic and septic ATN are the most common causes of intrinsic AKI, however, ATN can result from drug toxicities (2). AGN can be the result of autoimmune diseases, such as Lupus Nephritis, which can damage and inflame the glomeruli of the kidneys (2). AIN manifests as severe inflammation of the kidneys. This inflammation is usually the result of medications, such as antibiotics or a nonsteroidal anti-inflammatory drugs (NSAIDs) such as naproxen or ibuprofen (2). Additionally, AIN can be caused by streptococcal, viral, or Legionella infection (2). Post-renal AKI is commonly induced by the obstruction of the ureters, bladder outlet, or urethra (2). These obstructions may be due to prostatic conditions, anticholinergic agent therapy, blood clots, or ureter fibrosis (2). From herein the focus of this dissertation will focus on drug-induced intrinsic AKI.

Drugs are the third to fifth leading cause of AKI in critically ill patients (6) consistent with the kidneys' role in concentrating and excreting toxic metabolites and drugs (7), making them a common site of drug toxicity. Drugs damage the kidneys by several mechanisms: [1] vasoconstriction, [2] altered intraglomerular hemodynamics, [3] tubular cell toxicity, [4] interstitial nephritis, [5] crystal deposition, [6] drug-induced thrombotic microangiopathy, [7] osmotic nephrosis, and [9] rhabdomyolysis.

Vasoconstriction is the mechanism of nephrotoxicity of calcineurin inhibitors, vasopressors, and contrast agents (7). The use of NSAIDs, angiotensin converting enzyme inhibitors (ACEI), and angiotensin receptor blockers (ARB) 
can lead to altered intraglomerular hemodynamics resulting in a decline in kidney function (7). The proximal tubule is particularly vulnerable to toxic injury due to its role of reabsorbing the glomerular filtrate. Tubular cell toxicity is seen in a dosedependent manner for many drugs (e.g. aminoglycosides, amphotericin, calcineurin inhibitors, cisplatin, methotrexate, and antivirals) causing kidney injury (7), while crystal deposition in the distal tubular lumen is $\mathrm{pH}$-dependent and commonly seen in nephrotoxicity associated with acyclovir, sulfonamide, methotrexate, indinavir, and triamterene (7). Additionally, uric acid and calcium phosphate crystals are observed following chemotherapy (7). Drug-induced thrombotic microangiopathy has been reported with mitomycin, cyclosporin, tacrolimus, interferon, and quinine (7). Osmotic nephrosis has been associated with high doses of mannitol and immunoglobulins (7) and results from hyperoncotic solutions by decreasing glomerular filtration rate (GFR) or from osmotically induced tubular damage (7).

Unfortunately, the need for many of these medications outweighs the risk of nephrotoxicity development. During these instances measures are taken to minimize the drug-induced kidney damage, but these measures are not always successful. For these reasons innovative and novel research is needed toward the prevention of drug-induced AKI.

\section{History of cisplatin in the clinic}

Cisplatin is a widely used chemotherapeutic to treat a variety of solid malignant tumors (e.g. head and neck, ovarian, testicular, and lung cancer) (8). 
As discussed previously, cisplatin is associated with proximal tubule toxicity in AKI. To fully understand cisplatin's efficacy in the clinic despite its toxicity, it is important to also understand its development and long history of use clinically.

The biological effects of platinum were discovered in 1961 by a physicist, Barnett Rosenberg, while examining if alternating electromagnetic forces could affect cell division (9). Rosenberg set up platinum conducting plates in a chamber with Escherichia coli (E. coli) grown on a medium containing ammonium chloride, which provided the nitrogen required for cell growth (9). Rosenberg then applied an electric current and observed that the E. coli stopped dividing (9). He carried out further experiments to determine what was inhibiting the cell division, and discovered it was the platinum when combined with ammonia molecules (9). This discovery led to the further development of using platinum complexes as antitumor agents. A number of platinum complexes were tested, however, only the "cis" complexes with adjacent ammonia groups displayed anti-tumor activity (9). In 1969 the cisplatin (cis-diammine-dichloroplatinum (II)) compound was discovered to have the most potent anti-tumor effects (9). Cisplatin has a central platinum atom bound by ammonia and chloride groups in a square planar structure (10) (Figure 1.3). Cisplatin forms DNA crosslinks which interrupt cellular DNA functioning and induces apoptosis (10). This is accomplished through the two "leaving" chloride groups which readily dissociate in physiological conditions, and the subsequent formation of a platinum-DNA adducts that can only be repaired using nucleotide excision repair (10). 


\section{Figure 1.3}

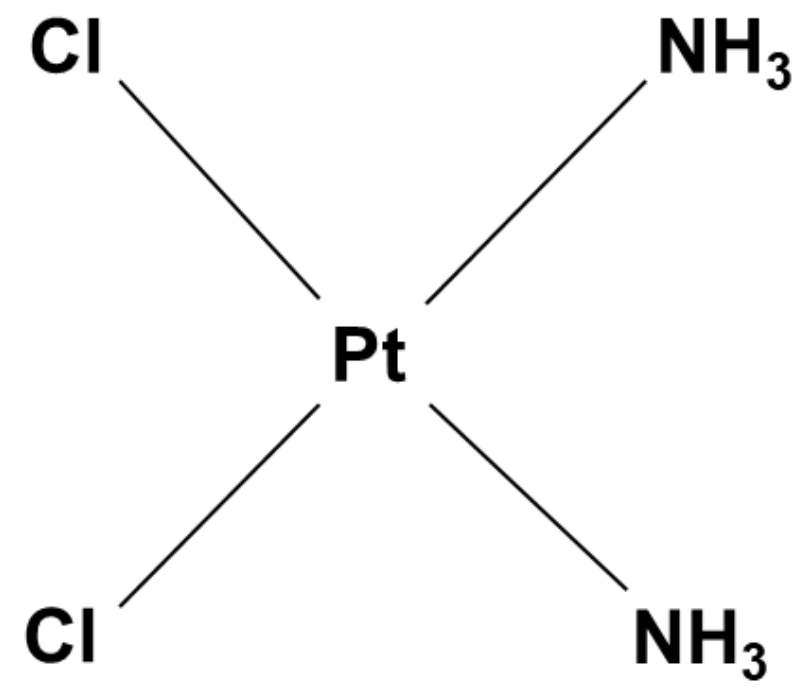

Figure 1.3: Structure of cis-diamminedichloroplatinum (II) or cisplatin.

Molecular structure of cisplatin is a square planar complex with two labile chlorine and two inert ammonia molecules that are coordinated to the central platinum (II) atom in the cis configuration. 
Following pre-clinical and human studies cisplatin was approved by the Food and Drug Administration (FDA) in 1978 for the treatment of advanced metastatic testicular, ovarian and bladder cancer (11). Since then it has had great success as a chemotherapeutic agent and is one of the most widely used anti-tumor drugs in the world. For example, in the early 1970s metastatic testicular cancer was associated with a $5 \%$ survival rate (12). Improved surgical techniques in combination with cisplatin chemotherapy has increased the 5-year survival rate for men with testicular cancer to 95\% (12). Furthermore, as recently as 2010 there were over 500 active clinical trials involving cisplatin (13), underlying its continued prominent use in the treatment of solid malignant tumors. Unfortunately, the major limiting factor of cisplatin is its nephrotoxicity.

The first report of cisplatin's nephrotoxicity was in 1971 during pre-clinical animal studies (13). These studies found histopathologic changes of ATN along with azotemia (abnormally high levels of nitrogen containing compounds in the blood). Early clinical use of cisplatin saw dose-related renal failure in $14-100 \%$ of patients, with the incidence varying with the cumulative dose (13). This high severity of toxicity almost prevented cisplatin's FDA approval as a chemotherapeutic agent $(8,14)$. In response, hydration protocols were developed that reduced the nephrotoxicity and allowed the implementation of increased doses to reach therapeutic levels $(8,15)$. Subsequently, cisplatin alone and in combination based chemotherapy regimens are currently used as a frontline therapy for testicular cancer, ovarian germ cell tumors, epithelial ovarian 
cancer, head and neck cancer, advanced cervical cancer, bladder cancer, mesothelioma, endometrial cancer, non-small cell lung cancer, malignant melanoma, carcinoids, penile cancer, and adrenocortical carcinoma (8). Additionally, it is used as a consolidation therapy for many types of solid tumors that have failed standard treatment regimens (8). Even though cisplatin is widely used, and hydration protocols decreased the incidences of kidney injury, cisplatin is still associated with a risk of $20-30 \%$ of patients developing AKI after a single dose. Research has been ongoing to both understand how cisplatin damages the kidney and to discover preventative methods.

\section{Cisplatin and the kidney}

Nephrotoxicity is an unusual side effect of a chemotherapeutic agent in general. In most cases chemotherapy drugs target pathways that are essential for cell division and therefore rapidly dividing cells such as bone marrow cells are most sensitive to these agents. Thus, the high rate of nephrotoxicity in the kidney which as a low cell turnover rate is unexpected and indicates that cisplatin may have two distinct mechanisms that kill cells, one for highly prolific cancer cells and one for quiescent kidney cells.

Cisplatin is cleared by the kidney via glomerular filtration and tubular secretion $(13,16)$. Concentration of cisplatin is higher in the kidney than in the blood suggesting there is active accumulation of the drug by renal parenchymal cells $(8,13)$. Even though cisplatin was developed over 50 years ago, its mechanism of toxicity is not completely understood. Previous studies provided 
evidence that cisplatin undergoes a basolateral-to-apical transport within the kidney $(13,17)$. In more recent years studies have identified two membrane transporters that are capable of cisplatin uptake: copper transporter 1 (Ctr1) and organic cation transporter 2 (OCT2) (13). Both Ctr1 and OCT2 are highly expressed in the kidney and found in the basolateral membrane of the proximal tubule (Figure 1.4). Downregulation of Ctr1 protein expression in kidney cells decreases cisplatin uptake within the kidney $(13,18)$. Likewise, OCT2 knock out (KO) mice demonstrate significantly reduced cisplatin urinary excretion and nephrotoxicity $(13,19,20)$. Furthermore cimetidine, an OCT2 substrate, also reduced cisplatin uptake and cytotoxicity in vitro $(18,21)$, and patients with a nonsynonymous single-nucleotide polymorphism (SNP) in the OCT2 gene (rs316019) demonstrated a reduced cisplatin-induced nephrotoxicity $(13,19,20)$. Another membrane protein, the multidrug and toxin extrusion 1 (MATE1) protein, is responsible for cisplatin extrusion out of the proximal tubule into the tubular lumen (Figure 1.4). MATE1 KO mice also exhibited enhanced cisplatin nephrotoxicity (22). Taken together these studies suggest that the cell mechanisms for cisplatin transport in the proximal tubule play a critical role in determining the susceptibility to injury. In addition to renal cisplatin uptake, there is evidence that cisplatin undergoes a biotransformation to a more potent toxin in the kidney (Figure 1.4). Studies in rats and mice show that the process may begin with the formation of glutathione-conjugates in the circulation, possibly mediated by glutathione-S-transferase $(13,23,24)$. In the kidney these glutathione-conjugates are extruded by MATE1 and further cleaved to cysteinyl- 


\section{Figure 1.4}

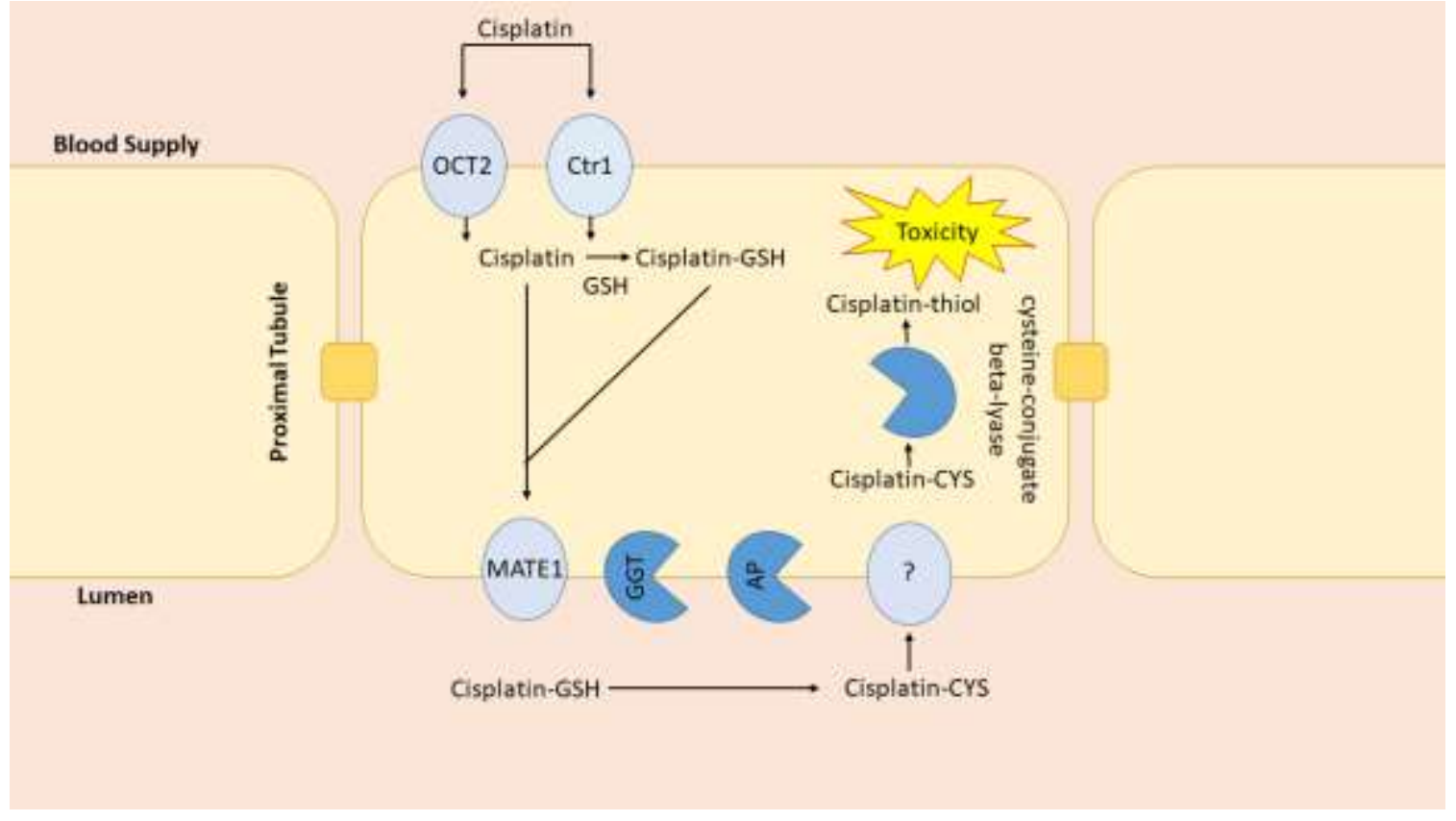

Figure 1.4: Cisplatin metabolism to a nephrotoxin.

Cisplatin enters the renal proximal tubule cell and is conjugated to glutathione (GSH). MATE-1 is responsible for exodus of cisplatin and cisplatin-glutathione conjugates into the tubule lumen. $\gamma$-glutamyl transpeptidase (GGT) and aminodipeptidase (AP) further metabolize cisplatin-glutathione conjugates to cisplatin-cysteinyl and cysteine conjugates. The cisplatin-cysteine conjugate reenters the renal proximal tubule cell and cysteine-S-conjugate beta-lyase metabolizes it to an unstable and reactive cisplatin-thiol conjugate, the nephrotoxic metabolite of cisplatin. 
glycine-conjugates by Y-glutamyl transpeptidase (GGT) (Figure 1.4), expressed apically in the proximal tubule $(13,25,26)$. The cysteinyl-glycine-conjugates are further metabolized to cysteine-conjugates by aminopeptidases (AP) (Figure 1.4), also expressed apically $(13,25,26)$. The cysteine-conjugates are transported back into the proximal tubule by an unknown protein transporter where they are further metabolized by cysteine-S-conjugate beta lyase (CCBL), forming a highly reactive thiol $(13,25-27)$ (Figure 1.4). This reactive thiol is what is believed to cause the toxicity. Experiments show that blocking any of these enzymes ameliorates cisplatin nephrotoxicity.

Renal tissue damage, characterized by tubular cell death, is a frequent and common occurrence of cisplatin nephrotoxicity. Subsequently renal tubule cell death may be in the form of apoptosis and necrosis. Necrotic cell death has been observed with high concentrations of cisplatin, whereas lower concentrations led to apoptosis in vitro $(12,28)$. In animal models both apoptosis and necrosis can be observed following cisplatin administration $(12,29)$. Although there has been a great deal of investigation into the mechanism of cisplatin-induced nephrotoxicity there are still many unanswered questions. As a result, it is still not understood why some patients are more susceptible to cisplatin nephrotoxicity. To develop either renoprotective strategies or therapies more research is needed regarding mechanisms of susceptibility along with identifying novel targets that do not affect cisplatin's efficacy. One such target recently discussed is the $\mathrm{Na} / \mathrm{H}$ exchange regulatory factor 1 (NHERF1) scaffolding protein. 


\section{NHERF1: is it more than an anchor?}

Cells communicate through coordinated signaling networks that rely on scaffolding proteins, which organize multiprotein complexes and enable proper transmission of information. NHERF1 is a member of a post-synaptic density protein 95/Drosophila Discs Large/Zonula Occulens -1 (PSD-95/DIg/ZO-1) homology (PDZ) scaffolding protein family. NHERF1 has two tandem PDZ domains, a carboxy terminal ezrin binding domain, and a CRAC (cholesterol binding) domain. Initially NHERF1 was identified (30) as an organizer and regulator of hormone receptors, signal transduction pathways, and transporters through its interaction with actin-binding-ezrin-radixin-moesin (ERM) proteins on the apical side of epithelial cells $(31,32)$. NHERF1 has many known functions in the kidney including: regulating the sodium hydrogen antiporter 3 (NHE3) function via the parathyroid hormone (PTH), regulating and anchoring the sodium phosphate co-transporter type-2 (Npt2a) expression by PTH and dopamine, and regulating the sodium pump. More recently NHERF1 has been found to be involved in a variety of functions across multiple cell types including: cell structure and trafficking, tumorigenesis and tumor behavior $(33,34)$, inflammatory responses $(2,18)$, and tissue injury $(35-37)$. There have been a number of divergent and contradictory reported effects of NHERF1, making it difficult to predict how either the absence or the overexpression of NHERF1 will affect a specific cell function.

\section{Cell Structure}


NHERF1 plays a role in actin cytoskeleton organization (38), cell polarity (39), and trafficking (40) possibly though its ability to scaffold signaling complexes via its interaction with ERM proteins. NHERF1 disrupts the actin skeleton in HeLa cells, thought to be through increased $\alpha$ actinin IV ubiquitination and degradation (38); however, in Madin-Darby-canine-kidney (MDCK) cells NHERF1 enhances actin skeleton organization (41) stimulated by podocalyxin-induced RhoA activation through the Rho/Rho-associated coiled-coil protein kinase (ROCK) pathway (42).

There are multiple functional consequences of the actin cytoskeleton organizational role of NHERF1. NHERF1 colocalization with proteins has been implicated in cell polarity through tight junction formations (39) and protein targeting to the apical or basolateral membrane (43-45). NHERF1 has a significant role in protein anchoring and assembly of multi-protein complexes. For example, NHERF1 expression is critical for assembling G-protein-coupled receptors (GPCRs), downstream signaling molecules (e.g. adenylyl cyclase), and target proteins such as Npt2a and the NHE3 in the proximal tubule. Additionally, NHERF1 is essential for regulating total body phosphate homeostasis by anchoring Npt2a through ezrin binding and subsequently the NHERF1 KO mouse displays phosphaturia and uricosuria (46).

In addition to membrane targeting NHERF1 may also play a role in establishing cell phenotype. NHERF1 mediated plasma membrane expression of the calcium adenosine triphosphatase (Ca-ATPase) 2 prevented involution of 
lactating mammary gland cells through suppression of the signal transducer and activator of transcription 3 (Stat3) activation (43).

\section{Cancer}

NHERF1 exhibits a dual role in tumorigenesis that is dependent on its expression or subcellular localization. When NHERF1 is located at the plasma membrane it acts as a tumor suppressor (47). In contrast, NHERF1 expression loss, cytoplasmic or nucleus localization results in pro-oncogenic properties (47). This phenomenon has been observed in melanoma (48), breast (49-54), reproductive system (55-58), digestive system (59-63), liver (64-66), lung (67), and brain cancers (68-71). Potential mechanisms for the effect of NHERF1 on tumor behavior include: inhibition of the epidermal growth factor receptor (EGFR) signaling due to a decreased interaction with NHERF1 (72), increased cytoplasmic expression of vascular endothelial growth factor (VEGF) and its receptor VEGFR1 (73), alteration in mineral metalloproteinase expression (74), and enhanced Wnt1 signaling (75). Furthermore, NHERF1 overexpression has been linked in liver cancer cells, regulation of cell cycle $(45,76,77)$, and suppression of reactive oxygen species (ROS) (78).

Cytoplasmic localization of NHERF1 has been implicated in the development of metastatic cancers (79). A potential mechanism may involve differential phosphorylation of NHERF1, where NHERF1 creates protein complexes which either activate or inactivate the promotion of metastatic behavior (80). For instance, overexpression of NHERF1 has been associated with pro-metastatic behavior in breast cancer cell lines. Moreover, overexpression of NHERF1 was 
connected with enhanced cell functions associated with the metastatic phenotype and mitigated by mutations of the phosphorylation sites S279 or S301 in NHERF1. NHERF1 phosphorylation is also essential for tumor-induced angiogenesis (80).

\section{Inflammation and fibrosis}

ROS impacts aging/fibrosis, cancer, neurodegenerative disorders, and cardiovascular disease (81). Interestingly, NHERF1 has been found to be a binding partner and regulator of NADPH oxidase (Nox1) by directly associating with the Nox organizing subunit $47^{\text {phox }}$. Additionally, NHERF1 interacts with other proteins involved in inflammatory pathways including the interleukin 8 (IL8) receptor beta, C-X-C Motif light-chain-enhancer of activated B cells (NF-kB), and simultaneously increased NF-кB production.

A study using a rat aging model found NHERF1 expression loss was associated with renal fibrosis development (84). One potential mechanism is suggested by the observation that NHERF1 blunted the transforming growth factor beta 1 (TGF- $\beta 1$ ) expression of mesenchymal cell markers in a lung cancer cell line (85). Additionally, a role for NHERF1 in regulating epithelialmesenchymal transition (EMT) is another possibility in lieu of previously cited studies where NHERF1 plays a role in cell polarity, cell phenotype expression, and cell cycle regulation.

\section{Response to injury}

NHERF1 expression has been implicated in responses to injury. In a model of cholestatic liver injury induced by bile duct ligation the NHERF1 KO mouse 
exhibits attenuated liver injury (36). Loss of NHERF1 also resulted in the deletion of the ICAM-1-ERM-NHERF1 complex resulting in a reduction in both hepatic ICAM1 and ERM proteins, which are essential for neutrophil mediated cholestatic liver injury (36). Therefore, NHERF1 may have a sensitizing role in inflammatory induced liver injury. On the other hand, NHERF1 loss does not result in protection against cisplatin-induced AKI. In fact NHERF1 KO mouse kidneys were more susceptible to cisplatin-induced AKI (35), exhibiting a decrease in renal function, a significant increase in urine neutrophil gelatinase-associated lipocalin (NGAL), and severe histologic damage (casts, BBM sloughing, and ATN) when compared to cisplatin treated wild type (WT) mice.

Interestingly the absence of NHERF1 protected against liver tissue injury in the biliary ligation model, but increased sensitivity to injury in the cisplatininduced AKI model. Thus, NHERF1 may have a significant role in the severity of injury after specific insults and in specific tissues potentially due to regulation of the acute inflammatory response.

\section{Implications for renal physiology/pathophysiology}

NHERF1 has a much broader role than as a scaffold or anchor in the cell, illustrated by the effect NHERF1 has on a variety of diseases and tissues along with its contributions to cell growth and proliferation, cell polarity and structure, determination of cell phenotype, response to inflammation, and neoplastic transformation. For this dissertation the role NHERF1 plays in susceptibility to cisplatin-induced AKI (35) will be further investigated. However, many questions remain including: whether NHERF1 is a primary mediator or responder of kidney 
injury and if NHERF1 can be used as a biomarker or therapeutic target for the prevention of kidney injury.

\section{Metabolism and susceptibility to cisplatin toxicity}

As previously discussed, the accumulation and bioactivation of cisplatin to a more potent nephrotoxic metabolite underlies the kidney's susceptibility to cisplatin-induced AKI. Recent studies have found that cisplatin alters renal cell metabolism, contributing to injury and the common occurrence of CKD development.

Several studies have found that cisplatin treatment leads to renal tubular cell depletion of amino acids, caused by both elevated levels of aminoaciduria and decreased tubular reabsorption of amino acids (86-89). Interestingly, branchedchain amino acids such as leucine, isoleucine, and valine were found to be elevated in urine and decreased in serum $(86,90)$. Many have discussed if this phenomenon could be used as either a biomarker for prediction of susceptibility to nephrotoxicity or enable earlier detection of kidney injury following cisplatin treatment (86). Moreover, cisplatin influences lipid metabolism through the reduction of fatty acid oxidation resulting in accumulation of fatty acids in kidney tissue $(86,89,91)$. Likewise, diacylglycerols, triacylglycerols, ceramides, and neutral lipids accumulate in kidney tissue $(86,87,92)$. Cisplatin also impacts glycolysis through the reduction of glycolytic enzymes, thus, glucose will accumulate while metabolites of glycolysis decrease $(86,93)$. In addition to 
glycolysis, cisplatin also decreases intermediates of the pentose phosphate pathway and the citric acid cycle $(86,94)$.

These observations bring into question if susceptible patients could have an altered renal cell metabolism, consequently, predisposing these patients to nephrotoxic injury from cisplatin. This hypothesis will be further discussed and investigated later in this dissertation.

\section{Mitochondrial dysfunction and susceptibility to cisplatin toxicity}

It has been well established that cisplatin nephrotoxicity induces cell death via both apoptosis and necrosis. However, the mechanisms by which cisplatin induces these pathways remains unclear. Recent evidence reveals that ROS and mitochondrial function play an important role in cisplatin's mechanism of injury (95). Mitochondria continuously produce ROS, such as superoxide, and scavenge these ROS using antioxidant enzymes (superoxide dismutase, glutathione peroxidase, catalase, and glutathione S-transferase) (96). Moreover, cisplatin has been found to also accumulate in the mitochondria of renal epithelial cells $(97,98)$. Several studies show that cisplatin induces ROS in renal epithelial cells by decreasing the activity of antioxidant enzymes along with the depletion of intracellular glutathione (GSH) concentrations (99-102). Furthermore, there are additional studies that demonstrate that pre-treatment with antioxidants such as GSH is beneficial in reducing cisplatin's nephrotoxicity $(103,104)$.

However, other studies suggest that at least in cultured proximal tubule cells, the primary cause of cell death post cisplatin treatment may be far more related 
to mitochondrial dysfunction than ROS formation $(100,105)$. Several of these studies show that cisplatin induces mitochondrial dysfunction, primarily through targeting enzymatic complexes that comprise the electron transport chain (ETC) $(100,105)$. Once the ETC is compromised, proximal tubule cells show a significant reduction in ATP, with a rapid metabolic collapse and necrotic cell death $(28,106)$. Additionally, slighter amounts of ATP depletion in tubule cells have been associated with lower doses of cisplatin $(106,107)$. Lower amounts of ATP depletion lead to apoptosis through the release of mitochondrial cytochrome c in tubule cells $(106,107)$. Similarly, to other aspects of cisplatin injury, the mechanism by which cisplatin causes cytochrome c release remains controversial. Two proposed mechanisms include induction of mitochondrial permeability transition (107) and increases in mitochondrial transmembrane potential (106). Likewise, the mechanism behind cisplatin's ability to inhibit the enzymatic complexes of the ETC are also unclear.

If ATP depletion and mitochondrial dysfunction play a central role in cisplatininduced cell death, then how does one explain the supporting evidence for ROS formation triggering cell death following cisplatin exposure? One possibility is that these two processes co-exist, and are not mutually exclusive in regards to cisplatin-induced cell death (8). It is possible that while the amount of ATP depletion may induce the primary cell injury and influence the cell death pathway, it may also accelerate ROS formation from the damaged cells (8), thus, contributing to an amplification loop leading to ROS mediated cell death (8). Especially since reduction in ROS through the pre-treatment of antioxidants can 
alleviate nephrotoxicity $(103,104)$, this may limit this amplification loop and reduce the accompanying inflammatory response (100).

Collectively, it is evident that both ROS production and mitochondrial dysfunction remain conceivable mechanisms of cisplatin-induced renal cell death. With this knowledge it brings into question if renal cells containing higher amounts of ROS and/or mitochondrial dysfunction could prime these cells for a 'second hit' with cisplatin, therefore, sensitizing these cells to cisplatin nephrotoxicity and possibly clarifying why some patients are more susceptible to cisplatin-induced AKI. This proposed mechanism will be further investigated throughout this dissertation.

\section{Statement of goals}

It is well established that cisplatin's limiting factor is its nephrotoxicity, but the mechanism of toxicity remains uncertain. Moreover, there are no methods to predict which patients are susceptible to injury and there are no targeted therapies to halt or reverse the development of AKI. Development of such methods or therapies requires a better understanding of mechanisms of disease progression as well as identification of novel drug targets. Therefore, one goal of this dissertation is to shed new insight into mechanisms of susceptibility to cisplatin-induced AKI. Additionally, it has been established that loss of the NHERF1 protein in mice predisposes these animals to cisplatin nephrotoxicity (35). Another goal of this dissertation is to elucidate mechanisms by which NHERF1 loss affects the kidneys. Taken together, the goal of this dissertation is 
to both understand the role NHERF1 plays in cisplatin nephrotoxicity and to speculate on its potential use as a biomarker and/or therapeutic target.

\section{B. Aims and proposals}

\section{Characterization of metabolic differences in kidneys of WT and NHERF1 KO mice}

As discussed in earlier sections, cisplatin significantly alters areas of metabolic function and cellular homeostasis. In addition, mitochondria are essential for maintaining this homeostasis. Mitochondria are responsible for ATP production and in regulating the cell death pathways of apoptosis and necrosis. Our laboratory has also established that loss of the NHERF1 scaffolding protein predisposes mice to cisplatin-induced AKI (35). Understanding the mechanism of susceptibility of these animals may be beneficial in understanding disease development of cisplatin-induced AKI along with potentially providing future therapeutic targets. Preliminary data from our laboratory suggested that NHERF1 KO mice may have an altered metabolic function. This was in part supported by proteomic analysis of the brush border membrane (BBM) of WT and NHERF1 KO mouse kidneys. This analysis showed notable decreases in mitochondrial proteins in the NHERF1 KO BBM. Furthermore, Seahorse XF24 analysis of isolated proximal tubule cells from WT and NHERF1 KO mouse kidneys exhibited a decrease in oxygen consumption rate (OCR). These results suggest that metabolic changes may play an important role in the sensitization of the 
NHERF1 KO kidney to insult. This is supported by the following: [1] NHERF1 deficient cells do not grow as rapidly as normal proximal tubule cells, suggesting that these cells are stressed. [2] NHERF1 KO mice treated with cisplatin display a significant increase in BUN levels that are consistent with a more severe renal injury when compared with treated WT mice (35). [3] Semi-quantitative scoring in a blinded study showed cisplatin treated NHERF1 KO kidneys have a higher level of severity in injury based on number of tubular casts, degree of ATN, and BBM sloughing (35). [4] Levels of apoptosis are similar between cisplatin treated WT and NHERF1 KO kidneys, indicating that damaged NHERF1 KO kidneys may have a higher level of ATP depletion resulting in higher levels of necrotic cell death (35). [5] Anaerobic glycolysis, a hallmark of AKI, is known to increase in rodent models treated with cisplatin (108) and in models of ischemic reperfusion (109) concomitant with a decrease in aerobic metabolism. The goal of this aim was to define any metabolic differences between kidneys of WT and NHERF1 KO mice. This study evaluated gluconeogenic, glycolytic, and pentose phosphate enzyme activity along with measuring ATP content of WT and NHERF1 KO mouse kidneys under basal and cisplatin treated conditions.

\section{Identification of changes in mitochondrial function in NHERF1 KO} mice kidneys

Cisplatin is known to accumulate in renal cell mitochondria and can damage mitochondrial enzymatic complexes. Additionally, mitochondrial dysfunction is one potential mechanism of cisplatin-induced renal cell death. It was 
hypothesized that an underlying mitochondrial dysfunction in NHERF1 KO kidneys may lead to sensitization of cisplatin nephrotoxicity. Furthermore, two proposed potential causes of mitochondrial dysfunction were: [1] Decrease in mitochondrial OCR also known as oxidative phosphorylation and/or alterations in mitochondrial structure in NHERF1 KO kidneys. [2] Decrease in number of mitochondria in NHERF1 KO kidneys. Therefore, electron microscopy (EM) was utilized to analyze both the mitochondrial morphology, distribution, and number of mitochondria in WT and NHERF1 KO kidneys. Additionally, mitochondria were isolated from WT and NHERF1 KO kidneys to evaluate function using the Seahorse XF24 Analyzer. This builds upon the work in Aim 1 of this dissertation, further evaluating metabolic and mitochondrial homeostasis as a potential mechanism of susceptibility to cisplatin nephrotoxicity in NHERF1 KO mice.

\section{Identification of mechanisms for enhanced cisplatin injury in NHERF1 KO mice}

Recently, a study showed that cervical cancer cells that are NHERF1 deficient have cisplatin resistance (110). Our laboratory previously established that NHERF1 KO mice have exacerbated cisplatin nephrotoxicity (35). Collectively, these data suggest that loss of NHERF1 may alter cisplatin cellular uptake and/or metabolism. Cisplatin metabolism begins with conjugation to GSH and within renal proximal tubule cells results in a nephrotoxic metabolite, cisplatin-thiol conjugate which is reactive and unstable and thus damages the mitochondria (111). Previous studies in mice and rats found that inhibition of 
GGT and CCBL, involved in cisplatin metabolism, blocked the nephrotoxic effects of cisplatin $(26,112,113)$. In in vitro studies, different cisplatin GSH conjugates led to more cell death to tubule cells than cisplatin alone (114). Aim 3 of this dissertation tests the hypothesis of potential changes in renal handling of cisplatin in NHERF1 KO mice. This was tested through the evaluation of lipid peroxidation, levels of small molecular weight thiols that act as antioxidants (e.g. GSH and Cysteine (Cys)), enzymatic activity of GGT, GGT and CCBL protein levels via western blot, and localization of GGT. Additionally, multiple time points (4 hours, 24 hours, and 72 hours) were evaluated based upon the established pharmacokinetics of cisplatin $(115,116)$.

\section{Overall aim of this dissertation.}

The overall aim of this dissertation is to provide new insight into mechanisms of susceptibility to cisplatin-induced AKI. To this end, the role of NHERF1 and sensitivity to cisplatin is a novel hypothesis that has not previously been investigated. Additionally, 3 potential mechanisms of susceptibility were investigated in this dissertation. [1] metabolic changes in NHERF1 KO mice kidneys (Aim 1) [2], mitochondrial function, morphology, and number in NHERF1 KO kidneys (Aim 2) [3], altered renal handling of cisplatin in NHERF1 KO kidneys (Aim 3). Taken together, this work will shed new mechanistic insight into cisplatin-induced AKI. 


\section{CHAPTER II}

\section{EXPERIMENTAL PROCEDURES}

\section{A. Animals and treatments}

Animal experiments were carried out by the Lederer laboratory. Mice were housed in a pathogen-free barrier facility, accredited by the Association for Assessment and Accreditation of Laboratory Animal Care. All animals used in these studies and procedures were in accordance with the guidelines established by University of Louisville's Institutional Animal Care and Use Committee (IACUC).

\section{Animal sacrifice, tissue collection, and storage}

At the time of sacrifice, animals were anesthetized with ketamine/xylazine (100/15 mg/kg, intraperitoneally (IP)). Blood was collected before sacrifice and plasma was extracted by centrifugation (10,000xg for 10 minutes). Plasma was stored at $-80^{\circ} \mathrm{C}$ until further analysis. Kidneys were removed and decapsulated. Portions of the kidney were snap-frozen in liquid nitrogen or immediately had the cortex separated from the rest of the kidney. The cortex was then either homogenized, frozen-fixed in Tissue Tek OCT-Compound (Sakura Finetek, Torrance, $\mathrm{CA}$ ) or immersed in $3.7 \%$ formaldehyde in phosphate buffered saline 
(PBS) for 24 hours followed by $70 \%$ ethanol prior to paraffin embedding for histology.

\section{Acute model of cisplatin nephrotoxicity}

Male (2-4 month old) WT C57BL/6J and C57BL6J NHERF1 ${ }^{(-/)}$KO mice (46) were maintained on a 12:12 hour light-dark cycle and were provided water and food ad libitum. Mice received a single IP injection of $20 \mathrm{mg} / \mathrm{kg}$ cisplatin (University of Louisville hospital pharmacy) or vehicle (saline). Vehicle treated and cisplatin treated mice were euthanized after 4, 24, and 72 hours. All studies were performed at the same time each day.

\section{B. Enzyme kinetics}

\section{Tissue preparation}

Kidney cortex was homogenized in $0.1 \mathrm{M}$ Tris- $\mathrm{HCl}, \mathrm{pH} 7.4$ on ice. Tissue homogenates were then sonicated on ice for 10 seconds/sample. The samples were then centrifuged for 15 minutes at $2,500 \times \mathrm{xg}$ at $4^{\circ} \mathrm{C}$.

\section{Fructose-1,6-Bisphosphatase activity assay}

Solutions of $0.1 \mathrm{M}$ Tris- $\mathrm{HCl}, \mathrm{pH} 8.6 ; 0.1 \mathrm{MgCl}_{2} ; 0.1 \mathrm{M}$ Cysteine $\mathrm{HCl} ; 50 \mathrm{mM}$ fructose-1,6-bisphosphatase (FBPase) were prepared. All components were added to tissue samples, except FBPase. Blanks were then prepared for the assay (solution blank and sample blank in solution). Each reaction was run in a 96 well plate with the FBPase added last to all samples and incubated for 60 
minutes at $37^{\circ} \mathrm{C} .10 \%$ trichloroacetic acid (TCA) was used to stop the reaction after the allotted time. Once a precipitate formed the plate was centrifuged at $10,000 \mathrm{xg}$ for 10 minutes to lower the precipitate to the bottom of the well. $5 \mathrm{~g}$ of $\mathrm{FeSO}_{4}$ was dissolved in $10 \mathrm{~mL}$ of $10 \%$ ammonium molybdate for working standards. $\mathrm{FeSO}_{4}$ working standards were prepared $(0,10,25,50,100,150,200$, $250 \mathrm{nM}$ ) and added to a new 96 well plate. For each sample $50 \mu \mathrm{L}$ of the first

reaction was then added to the new plate. Then $40 \mu \mathrm{L}$ of the $\mathrm{FeSO}_{4}$ solution was added last to each standard and sample well and incubated at room temperature for 10 minutes. The plate was read at 10 minutes at $820 \mathrm{~nm}$. (WT vehicle $\mathrm{n}=3$ ), (NHERF1 KO vehicle $n=4)$, (WT cisplatin $n=3)$, (NHERF1 KO cisplatin=5).

\section{Glucose-6-Phosphatase activity assay}

0.1 M Tris-HCl, pH 7.4; 10\% TCA; 50 mM glucose-6-phosphatase (G6Pase); $0.1 \mathrm{M} \mathrm{MgCl}_{2}$ were prepared. Additionally, a $3.2 \mathrm{mM} \mathrm{KH}_{2} \mathrm{PO}_{4}$ solution was prepared and stored at $4^{\circ} \mathrm{C}$. All working standards were prepared fresh using the 3.2 $\mathrm{mM} \mathrm{KH}_{2} \mathrm{PO}_{4}$ stock solution and diluted to $590 \mu \mathrm{M}$. Blanks were then prepared for the assay (solution blank and sample blank in solution). Each reaction was run in a 96 well plate with the G6Pase added last to all samples. The plate was then incubated for 60 minutes at $37^{\circ} \mathrm{C} .10 \%$ TCA was used to stop the reaction after the allotted time. Once a precipitate formed the plate was centrifuged at $10,000 \mathrm{xg}$ for 10 minutes to lower the precipitate to the bottom of the well. $5 \mathrm{~g}$ of $\mathrm{FeSO}_{4}$ was dissolved in $10 \mathrm{~mL}$ of $10 \%$ ammonium molybdate for working standards. $\mathrm{FeSO}_{4}$ working standards were prepared $(0,10,25,50,100,150,200$, 
$250 \mathrm{nM}$ ) and added to a new 96 well plate. Then $50 \mu \mathrm{L}$ of the $\mathrm{FeSO}_{4}$ solution was added last to each standard and sample well and incubated at room temperature for 10 minutes. The plate was read at 10 minutes at $820 \mathrm{~nm}$. (WT vehicle $n=3$ ), (NHERF1 KO vehicle $n=4)$, (WT cisplatin $n=3)$, (NHERF1 KO cisplatin=5).

\section{Lactate Dehydrogenase activity assay}

$0.1 \mathrm{M}$ Tris- $\mathrm{HCl}, \mathrm{pH}$ 7.4; $0.5 \mathrm{mM}$ sodium pyruvate; $0.1 \mathrm{M} \mathrm{MgCl}_{2}$ were prepared. Additionally, $0.5 \mathrm{mM} \mathrm{NADH}$ was made fresh in a $1 \mathrm{mM}$ sodium bicarbonate, $\mathrm{pH}$ 9.0 solution the day of the reaction. Blanks were then prepared for the assay (solution blank and sample blank in solution). Each reaction was run in a 96 well plate with the NADH added last to all samples. The plate was read immediately after adding the NADH at $340 \mathrm{~nm}$ for 3-10 minutes. (WT vehicle $\mathrm{n}=3$ ), (NHERF1 KO vehicle $n=4)$, (WT cisplatin $n=3)$, (NHERF1 KO cisplatin=5).

\section{Malate Dehydrogenase activity assay}

$0.1 \mathrm{M}$ Tris- $\mathrm{HCl}, \mathrm{pH} 7.4 ; 0.5 \mathrm{mM}$ oxaloacetate; $0.1 \mathrm{M} \mathrm{MgCl} 2$ were prepared. Additionally, $0.5 \mathrm{mM}$ NADH was made fresh in a $1 \mathrm{mM}$ sodium bicarbonate, $\mathrm{pH}$ 9.0 solution the day of the reaction. Blanks were then prepared for the assay (solution blank and sample blank in solution). Each reaction was run in a 96 well plate with the NADH added last to all samples. The plate was read immediately after adding the NADH at $340 \mathrm{~nm}$ for 3-10 minutes. (WT vehicle $\mathrm{n}=3$ ), (NHERF1 KO vehicle $n=4)$, (WT cisplatin $n=3),($ NHERF1 KO cisplatin=5). 


\section{Malic Enzyme activity assay}

$0.1 \mathrm{M}$ Tris- $\mathrm{HCl}, \mathrm{pH}$ 7.4; $0.5 \mathrm{mM} \mathrm{L-malic} \mathrm{acid;} 0.1 \mathrm{M} \mathrm{MgCl}_{2}$ were prepared. Additionally, $2 \mathrm{mg} / \mathrm{mL}$ of NADP was made fresh in a $100 \mathrm{mM}$ imidazole solution the day of the reaction. Blanks were then prepared for the assay (solution blank and sample blank in solution). Each reaction was run in a 96 well plate with the NADP added last to all samples. The plate was read immediately after adding the NADP at $340 \mathrm{~nm}$ for 3-10 minutes. (WT vehicle $n=3)$, (NHERF1 KO vehicle $n=4)$, (WT cisplatin $\mathrm{n}=3$ ), (NHERF1 KO cisplatin=5).

\section{Glucose-6-Phosphate Dehydrogenase activity assay}

$0.1 \mathrm{M}$ Tris- $\mathrm{HCl}, \mathrm{pH} 7.4 ; 0.5 \mathrm{mM}$ glucose-6-phosphate; $0.1 \mathrm{M} \mathrm{MgCl}_{2}$ were prepared. Additionally, $2 \mathrm{mg} / \mathrm{mL}$ of NADP was made fresh in a $100 \mathrm{mM}$ imidazole solution the day of the reaction. Blanks were then prepared for the assay (solution blank and sample blank in solution). Each reaction was run in a 96 well plate with the NADP added last to all samples. The plate was read immediately after adding the NADP at $340 \mathrm{~nm}$ for 3-10 minutes. (WT vehicle $\mathrm{n}=3$ ), (NHERF1 KO vehicle $n=4)$, (WT cisplatin $n=3),(N H E R F 1 K O$ cisplatin $=5)$.

\section{8. y-Glutamyl Transferase activity assay}

The activity assay was performed using the GGT colorimetric assay kit (Sigma Aldrich; St. Louis, Missouri) per the manufacturer's instructions. Tissue samples were prepared using approximately $10 \mathrm{mg}$ of kidney cortex in $200 \mu \mathrm{L}$ of ice-cold GGT assay buffer. The samples were then centrifuged at $13,000 \mathrm{xg}$ for 
10 minutes to remove insoluble material. The supernatant was kept and further

diluted to 1:100 using GGT assay buffer and stored at $-80^{\circ} \mathrm{C}$ until further use. 4 hours: (WT vehicle: $n=6),(N H E R F 1$ KO vehicle: $n=6),($ WT cisplatin: $n=6)$, and (NHERF1 KO cisplatin: $n=6$ ) and 24 hours: (WT vehicle: $n=5$ ), (NHERF1 KO vehicle: $n=5)$, (WT cisplatin: $n=7)$, and (NHERF1 KO cisplatin: $n=8)$.

\section{Liquid Chromatography-Mass Spectrometry (LC-MS)}

\section{LC-MS of kidney cortex for ATP quantification}

$10 \mathrm{mg}$ of kidney cortex was snap-frozen and stored in $-80^{\circ} \mathrm{C}$ until LC-MS procedure. The tissue was maintained in liquid nitrogen during preparation for LC-MS. Tubes and beads were pre-cooled in liquid nitrogen and $2.5 \%$ kidney cortex homogenate was made using an extraction solution $(70 \%$ acetonitrile and $30 \% \mathrm{H}_{2} \mathrm{O}$ ). Tissue was homogenized using a bead beater at $5 \mathrm{~m} / \mathrm{s}$ for 15 seconds. The homogenized tissue was then transferred to another tube for centrifugation at $16,000 \mathrm{xg}$ for $10 \mathrm{~min}$ at $4^{\circ} \mathrm{C}$. The supernatant was saved for subsequent LC-MS analysis. To quantitate the ATP present in mouse kidney cortex tissue a standard curve was prepared using $1 \mathrm{mM}$ ATP diluted serially with the extraction buffer containing $20 \mu \mathrm{M}{ }^{13} \mathrm{C}_{10} \mathrm{ATP}$. Each concentration point was diluted $50 \mathrm{X}$ with $60 \% \mathrm{ACN}$ and $40 \% 15 \mathrm{mM}$ Ammonium Acetate. $5 \mu \mathrm{L}$ was injected onto a SeQuant ZIC-cHILIC 100X2.1mm metal free HPLC column. Separation was performed by pumping $60 \% \mathrm{ACN}$ and $40 \% 15 \mathrm{mM}$ Ammonium Acetate through the column with a flow rate of $0.5 \mathrm{~mL} / \mathrm{min}$ with no change in composition using a Waters Acquity UPLC. The ATP was eluted from the column 
and flowed into a Waters Quattro Premier XE mass spectrometer and subsequently quantitated using optimized MRMs. A calibration curve was constructed using ATP concentration on the x-axis and response of ATP over ${ }^{13} \mathrm{C}_{10}$ ATP on the $y$ axis. Mouse kidney extracts were also diluted with $50 \mathrm{X} 60 \%$ ACN and $40 \% 15 \mathrm{mM}$ Ammonium Acetate, $5 \mu \mathrm{L}$ was injected and the concentration in kidney tissue was interpolated using the calibration curve previously made for $(\mathrm{WT} n=5)$ and (NHERF1 KO $n=5)$.

\section{Electron Microscopy}

\section{Perfusion fixation of total kidney in situ for electron microscopy}

The abdominal cavity of the mouse was opened along the linea alba and intestines moved aside to expose kidneys. A suture was loosely put around the right kidney for removal without glutaraldehyde exposure. The right kidney was then used for kidney cortex homogenates. Next, the chest cavity was opened to expose the heart and the vena cava was cut while the right kidney was tied off and removed. The left mouse kidney was perfused with $3 \%$ glutaraldehyde solution at a rate of $6 \mathrm{~mL} / \mathrm{min}$ for approximately $1-3$ minutes. Perfusion was stopped once kidneys had a change in color and consistency. The kidney was removed and placed in a petri dish of $3 \%$ glutaraldehyde where three to four 0.2 $\mathrm{cm} \times 0.4 \mathrm{~cm}$ slices were cut and then stored in 3-4 $\mathrm{mL}$ of $3 \%$ glutaraldehyde at $4^{\circ} \mathrm{C}$. At this point the tissue was sent to Norton Children's Hospital Pathology Department for a blinded EM analysis of kidney tubule mitochondria. Images were taken by a renal pathologist at the Pathology Department. Sections with the 
highest concentration of mitochondria were randomly picked in a $4 x$ field to be used to calculate mitochondria number and mitochondria area by Image J (WT $\mathrm{n}=6)$ and (NHERF1 KO $\mathrm{n}=5)$.

\section{E. Seahorse XF24 mitochondrial respiration analysis}

Mice for this study had food taken away 6 hours before sacrifice.

Mitochondrial oxidative capacity was measured in isolated kidney mitochondria using a Seahorse Bioscience XF24 extracellular flux analyzer (Billerica, MA, USA). For measurements in isolated mitochondria, tissue from the kidney cortex of both kidneys (approximately $50 \mathrm{mg}$ ) was isolated and homogenized in $1 \mathrm{ml}$ of isolation buffer (220 mM mannitol, $70 \mathrm{mM}$ sucrose, $5 \mathrm{mM} 3-(\mathrm{N}-$ morpholino)propanesulfonic acid (MOPS), $1 \mathrm{mM}$ ethylene glycol tetraacetic acid (EGTA), $0.3 \%$ fatty acid-free bovine serum albumin (BSA), $\mathrm{pH} 7.2$ ). The homogenate was centrifuged at $500 \times \mathrm{g}$ for five minutes at $4^{\circ} \mathrm{C}$. The supernatant containing mitochondria was centrifuged at $10,000 \times \mathrm{g}$ for five minutes. Following two wash centrifugation steps in BSA-free isolation buffer, the mitochondria were suspended in respiration buffer (120 mM KCl, 25 mM sucrose, 10 mM 4-(2hydroxyethyl)-1-piperazineethanesulfonic acid (HEPES), $1 \mathrm{mM} \mathrm{MgCl}$, $5 \mathrm{mM}$ $\mathrm{KH}_{2} \mathrm{PO}_{4}, \mathrm{pH}$ to 7.2$)$. Protein in the mitochondrial suspension was estimated by bicinchoninic acid method (Sigma) using BSA as a standard. $10 \mu \mathrm{g}$ of mitochondrial protein was sedimented in XF culture plates. Succinate (10 mM), rotenone $(1 \mu \mathrm{M})$, and ADP $(1 \mathrm{mM})$ were injected to assess state 3 respiratory activity (phosphorylating respiration). The OCR of mitochondria after exposure to 
oligomycin $(1 \mu \mathrm{g} / \mathrm{ml})$ was used to estimate state 4 activity (non-phosphorylating respiration). Finally, Antimycin $\mathrm{A}(20 \mu \mathrm{M})$ was stop all respiration. Data are expressed as $\mathrm{pmol} \mathrm{O} / \mathrm{min} / \mu \mathrm{g}$ protein (WT $\mathrm{n}=6$, NHERF1 KO $\mathrm{n}=6$ ).

\section{F. Histology and immunohistochemistry}

\section{Histology}

Paraffin embedded fixed whole kidney tissue were cut in $5 \mu \mathrm{m}$ thick either transversely or longitudinally from cisplatin treated and vehicle treated WT and NHERF1 KO animals. These sections were stained with hematoxylin and eosin (H\&E) as well as periodic acid-schiff (PAS) to evaluate number of casts, degree of BBM sloughing, and ATN. These sections were sent to a pathologist for analysis. Images were taken at 20x and 40x (WT vehicle $n=5$ ), (NHERF1 KO vehicle $n=5)$, (WT cisplatin $n=7)$, and (NHERF1 KO cisplatin $n=8)$.

\section{Immunohistochemistry (IHC)}

IHC was carried out on paraffin embedded fixed whole kidney tissue cut in 5 $\mu \mathrm{m}$ thick sections either transversely or longitudinally from cisplatin treated and vehicle treated WT and NHERF1 KO animals. A primary antibody against GGT (1:50) (ABclonal; Woburn, MA; A1776) and 4-Hydroxynonenal (4-HNE) (1:100) (Abcam; Cambridge, UK; ab46545) were utilized for staining to evaluate any changes in proximal tubule localization or loss after cisplatin treatment. Images were taken at 10x, 20x and 40x (WT vehicle: $n=5)$, (NHERF1 KO vehicle: $n=5$ ), (WT cisplatin: $n=10)$, and (NHERF1 KO: $n=9$ ). 


\section{G. Clinical Chemistry}

\section{Neutrophil gelatinase-associated lipocalin (NGAL)}

NGAL was determined using an enzyme linked immunosorbent assay (ELISA) kit (R\&D Systems; Minneapolis, MN; DY1857) on mouse urine as

directed by the manufacturer (WT vehicle $n=5)$, (NHERF1 KO vehicle $n=5)$, (WT cisplatin $n=7$ ), and (NHERF1 KO cisplatin $n=8$ ).

\section{H. Thiobarbituric acid reactive substances (TBARS)}

Lipid peroxidation was determined using a TBARS assay kit (Cayman

Chemicals; Ann Arbor, Ml; 10009055) on mouse kidney cortex tissue as directed by the manufacturer (WT vehicle $n=5)$, $($ KO vehicle $n=5)$, (WT cisplatin $n=8)$, and (NHERF1 KO cisplatin $n=9$ ).

\section{High-performance Liquid Chromatography (HPLC)}

\section{Collection of Mouse Blood and Tissue for GSH, GSSG, Cys, and Cyss}

Blood samples were collected from mice and added to a tube with $0.5 \mathrm{M}$ borate buffer stock solution and $165 \mathrm{uM} \mathrm{Y-glutamylglutamate}$ as the internal standard as described in (117) within 1-2 minutes after the blood was drawn. Plasma was extracted via centrifugation (3,000xg for 2 minutes) and $50 \mathrm{uL}$ of supernatant was transferred to a new tube with preservation solution $(10 \%$ perchloric acid, $0.2 \mathrm{M}$ boric acid, and $10 \mathrm{uM} \mathrm{Y-glutamylglutamate).} \mathrm{Tubes} \mathrm{were}$ then stored at $-80^{\circ} \mathrm{C} .10 \mathrm{mg}$ of kidney cortex tissue was removed from the kidney and added to preservation solution (5\% perchloric acid, $0.2 \mathrm{M}$ boric acid, and 10 
uM Y-glutamylglutamate) and homogenized. Kidney cortex homogenates were then stored at $-80^{\circ}$ until further use. Plasma: (WT vehicle: $\left.n=5\right)$, (NHERF1 KO vehicle: $n=5)$, (WT cisplatin: $n=7)$, and (NHERF1 KO cisplatin: $n=9$ ).

\section{HPLC Analysis of kidney cortex and plasma for GSH, GSSG, Cys, and Cyss}

Tissue and plasma Cys, CySS, GSH and GSSG were assayed by HPLC as S-carboxymethyl, $\mathrm{N}$-dansyl derivatives using Y-glutamylglutamate as an internal standard as previously described $(117,118)$. The HPLC system consisted of an aminopropyl column, 2 Waters 515 HPLC Pumps to deliver a gradient of acetate and methanol, a Waters 717 plus Autosampler with refrigeration unit, and a Waters 2475 Multi $\lambda$ Fluorescence Detector. Integration of peak areas and comparison to internal standard was performed by Waters Empower 3 software. Kidney: (WT vehicle: $n=5$ ), (NHERF1 KO vehicle: $n=5)$, (WT cisplatin: $n=8)$, and (NHERF1 KO cisplatin: $n=9$ ).

\section{J. Immunoblots}

\section{Western blot}

Kidney cortex homogenates were made as previously described (119).

Protein concentration was measured by bicinchoninic acid (BCA) method (Sigma) using BSA as a standard. Kidney homogenates were separated by $10 \%$ SDS-PAGE and transferred to nitrocellulose membranes. The membranes were incubated with $5 \%(\mathrm{wt} / \mathrm{vol})$ dried milk in Tris-buffered saline with $0.5 \%$ Tween 20 
(TTBS) for 30 minutes to decrease nonspecific antibody binding. Membranes were incubated overnight at $4^{\circ} \mathrm{C}$ with primary antibodies against GGT (ABclonal; A1776) and CCBL1 (ABclonal; A6542), diluted (1:1000) in 5\% milk. Membranes were washed with TTBS and incubated for 2 hours with horseradish peroxidaseconjugated secondary antibodies diluted in TTBS and 5\% milk.

Chemiluminescence (ThermoScientific) was utilized to detect bands and visualization via GeneSys software with a Pixi imaging system (Syngene). Densitometric analysis was performed via Image J. For densitometric analysis, specific protein expression was normalized to densitometry values for

glyceraldehyde 3-phosphate dehydrogenase (GAPDH) (Cell Signaling; Beverly, MA) or $\beta$-actin (Cell Signaling) in each lane (WT vehicle $n=3$ ), (NHERF1 KO vehicle $n=3$ ), (WT cisplatin $n=4)$, and (NHERF1 KO cisplatin $n=4)$.

\section{Statistical analysis}

1. Statistical analysis of ATP, mitochondrial number, and respiration data

Student's t-test was used to evaluate LC-MS data measuring ATP content between WT and NHERF1 KO kidneys. Additionally, student's t-test was used to compare mitochondrial number and mitochondrial respiration via Seahorse XF24 between WT and NHERF1 KO kidney mitochondria. $P$-values of $<0.05$ were considered statistically significant and data were shown as mean \pm standard error means (SEM). 


\section{Statistical analysis of enzyme kinetics, western blots, HPLC, NGAL,}

and TBARS

Two-way analysis of variance (ANOVA) was used to evaluate changes in enzyme kinetics between vehicle and cisplatin treated mouse kidneys. Likewise, two-way ANOVA was utilized to evaluate protein expression changes in GGT and CCBL in treated kidney cortex by western blot; reduction/oxidation state of GSH and Cys in plasma and treated kidneys; lipid peroxidation by TBARS in treated kidneys; and urine NGAL from treated mice. $P$-values of $<0.05$ were considered statistically significant and data were shown as mean \pm SEM. 


\section{CHAPTER III}

\section{NHERF1 LOSS RESULTS IN ALTERED PENTOSE PHOSPHATE ENZYMES IN MICE}

\section{A. Introduction}

Although cisplatin is a widely used chemotherapeutic that treats a variety of solid malignant tumors (e.g. ovarian testicular, head and neck, and lung cancer), its mechanism of action is still not completely understood (8). Likewise, the mechanism of cisplatin's nephrotoxicity remains perplexing and is surrounded by much controversy. Moreover, $20-30 \%$ of patients on cisplatin will develop cisplatin-induced AKI with a single dose (8). Currently, there are no methods of prevention for cisplatin-induced AKI or therapeutics to treat the development of AKI. Therefore, understanding patient susceptibility to cisplatin is a needed area of research.

Identifying new potential biomarkers and/or therapeutic targets are a promising area of research. To meet this need, a new area of research has been proposed to involve the scaffolding protein NHERF1 and susceptibility to cisplatin-induced AKI (35). NHERF1 is a known monomeric intrinsic membrane protein that belongs to the NHERF family of PDZ-scaffold proteins (31). NHERF1 
is found in all epithelial cells and acts as a scaffold for multi-protein signaling complexes. In proximal tubule cells of the kidney NHERF1 is anchored to the cytoskeleton in the subapical plasma membrane where it acts as a key scaffolding protein of transport proteins and has critical roles in defining the renal proximal tubule BBM composition and in regulating ion transport (31).

Furthermore, there is increasing evidence that NHERF1 has a much broader role than as a scaffolding protein. In fact, NHERF1 expression and/or localization has been associated with tumorigenesis $(33,34)$. Several studies have documented when NHERF1 is localized at the apical membrane it acts as a tumor suppressor, and when its expression is lost or its localization is in the cytoplasm or nucleus it exhibits pro-oncogenic properties $(33,34)$. Additionally, NHERF1 has received increased interest in its involvement in a variety of functions including: cell structure and trafficking $(38,40)$, inflammatory responses $(82)$, and tissue injury (35-37). One such study found that NHERF1 KO mice had attenuated cholestatic liver injury by bile duct ligation (36). NHERF1 loss has been reported in exacerbation of cisplatin-induced AKI in mice (35). Furthermore, aging rats (22 months) exhibit a decrease in NHERF1 expression (84) associated with the development of renal fibrosis.

Taken together, NHERF1 expression may also be lost in aging humans, predisposing these patients to cisplatin nephrotoxicity. One question addressed in this dissertation is whether NHERF1 loss may alter metabolic pathways resulting in increased sensitivity to cisplatin. Cisplatin itself is known to modify 
renal metabolic pathways such as glycolysis, gluconeogenesis, and lipid metabolism in response to cellular injury and death $(86,87,92,93)$.

\section{B. Experimental Procedures}

\section{Animals and treatments}

Male (2-4 month) WT and NHERF1 KO mice were treated with cisplatin (20 $\mathrm{mg} / \mathrm{kg} \mathrm{IP)} \mathrm{for} 72$ hours as described in Chapter II.

\section{LC-MS of kidney cortex for ATP quantification}

Snap-frozen kidneys were prepared for LC-MS analysis as described in Chapter II.

\section{Enzyme kinetics}

Kidney cortex tissue was homogenized and prepared for enzyme kinetic assays as described in Chapter II. Enzyme kinetic assays were run for FBPase, G6Pase, lactate dehydrogenase (LDH), malate dehydrogenase (MDH), malic enzyme, and glucose-6-phosphate dehydrogenase (G6PD) as described in Chapter II.

\section{Data analysis \\ Enzyme kinetic data were analyzed as described in Chapter II.}

\section{Statistical analysis}


Enzyme kinetic data were analyzed by two-way ANOVA as described in Chapter II.

\section{C. $\underline{\text { Results }}$}

\section{Cisplatin treatment significantly decreases fructose-1,6-} bisphosphatase enzyme activity in both WT and NHERF1 KO mice.

Previous studies investigated the effect of cisplatin on gluconeogenesis (86, $93,120)$. However, the effect of NHERF1 protein loss on gluconeogenesis before and after cisplatin treatment has not been investigated. The first objective of this dissertation was to measure the enzyme activity of FBPase before and after cisplatin treatment in WT and NHERF1 KO mice. FBPase is a critical regulatory enzyme in gluconeogenesis that catalyzes the hydrolysis of fructose-1,6bisphosphate to fructose-6-phosphate and inorganic phosphate (121). In order to determine if NHERF1 loss affected gluconeogenic enzyme activity alone or with cisplatin treatment, male 2-4 month old mice were treated with vehicle or cisplatin to induce $\mathrm{AKI}$ and then sacrificed after 72 hours. Kidney cortex tissue from these mice were used for the FBPase enzyme kinetic assay as described in Chapter II. There were no significant differences between vehicle [(WT: $41.5 \mathrm{nmole} / \mathrm{mg}$ protein/min \pm 6.5$)(\mathrm{KO}: 38.7 \mathrm{nmole} / \mathrm{mg}$ protein/min \pm 4.1$)$ ] or cisplatin [(WT: 20.6 $\mathrm{nmole} / \mathrm{mg}$ protein $/ \mathrm{min} \pm 0.4)(\mathrm{KO}: 19.7 \mathrm{nmole} / \mathrm{mg}$ protein $/ \mathrm{min} \pm 1.2)]$ treated WT and NHERF KO kidneys (Figure 3.1). However, cisplatin did decrease FBPase activity in both WT and NHERF1 KO kidneys $(P=0.0001)$ (Figure 3.1). 


\section{Figure 3.1}

Fructose-1,6-Bisphosphatase Activity

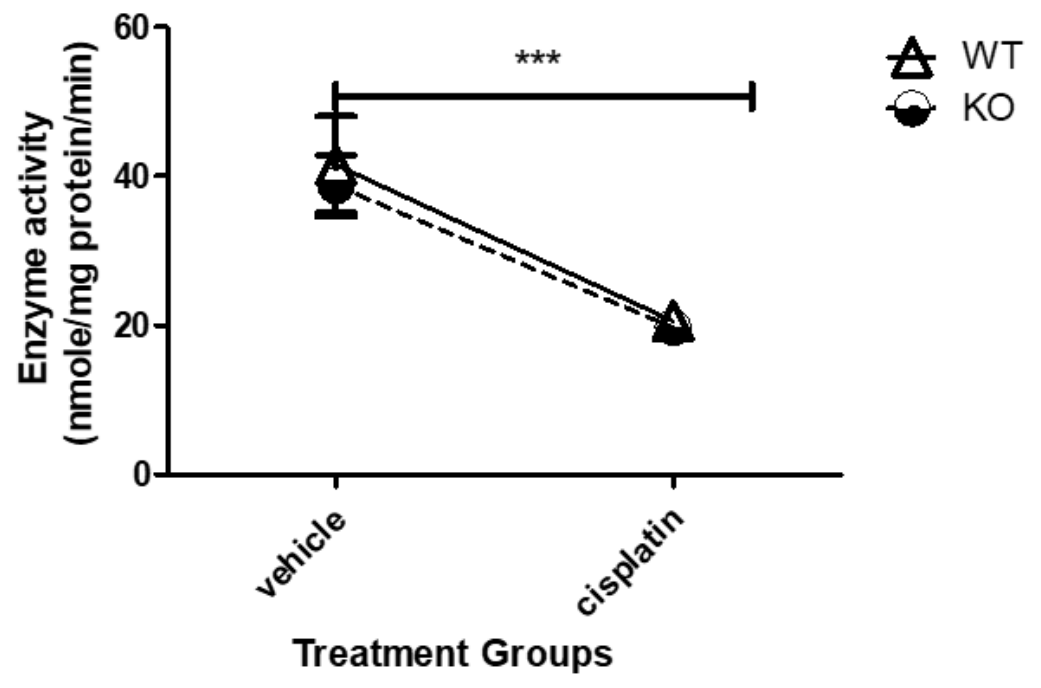

Figure 3.1: Effect of cisplatin treatment on fructose-1,6-bisphosphatase enzyme activity in WT and NHERF1 KO mouse kidneys.

2-4 month old male C57BL/6J WT and NHERF1 KO mice were given cisplatin (20 mg/kg dose IP) or vehicle (saline) and sacrificed after 72 hours as described in Chapter II. FBPase enzyme activity was determined from kidney cortex tissue of these mice. Data are means \pm SEM (WT vehicle $n=3)$, (KO vehicle $n=4)$, (WT cisplatin $\mathrm{n}=3$ ), and (KO cisplatin $\mathrm{n}=5) .{ }^{* *} P=0.001$ cisplatin treated WT and NHERF1 KO mice compared to vehicle saline controls. 


\section{Cisplatin treatment significantly decreases glucose-6-phosphatase}

enzyme activity in both WT and NHERF1 KO mice.

G6Pase is the final step in gluconeogenesis. It hydrolyzes glucose-6-

phosphate to free glucose and a phosphate group (122). This enzyme was also investigated to evaluate the effect NHERF1 loss may have on gluconeogenesis alone or with cisplatin treatment. Kidney cortex tissue from these mice were used for the G6Pase enzyme kinetic assay as described in Chapter II. Similarly to FBPase, G6Pase activity remained comparable in WT and NHERF1 KO kidneys regardless of treatment [(WT vehicle: $92.3 \mathrm{nmole} / \mathrm{mg}$ protein $/ \mathrm{min} \pm 5.0),(\mathrm{KO}$ vehicle: $103.0 \mathrm{nmole} / \mathrm{mg}$ protein/min \pm 11.3 ), (WT cisplatin: $38.0 \mathrm{nmole} / \mathrm{mg}$ protein/min \pm 5.1 ), and (KO cisplatin: $26.4 \mathrm{nmole} / \mathrm{mg}$ protein $/ \mathrm{min} \pm 3.8$ )], while cisplatin led to a significant decrease in enzyme activity in both genotypes ( $P$ $<0.0001$ ) (Figure 3.2).

\section{NHERF1 loss or cisplatin treatment does not significantly affect lactate dehydrogenase enzyme activity in mice.}

Lactate dehydrogenase (LDH) catalyzes the interconversion of lactate and pyruvate, concomitantly with the interconversion of $\mathrm{NADH}$ and $\mathrm{NAD}^{+}(123)$. When oxygen is absent LDH converts pyruvate, the final product of glycolysis, to lactate (123). Kidney cortex tissue from WT and NHERF1 KO vehicle and cisplatin treated mice were used to evaluate enzyme activity of LDH as described in Chapter II. NHERF1 loss $(P=0.65)$ or cisplatin treatment $(P=0.71)$ did not 


\section{Figure 3.2}

\section{Glucose-6-Phosphatase Activity}

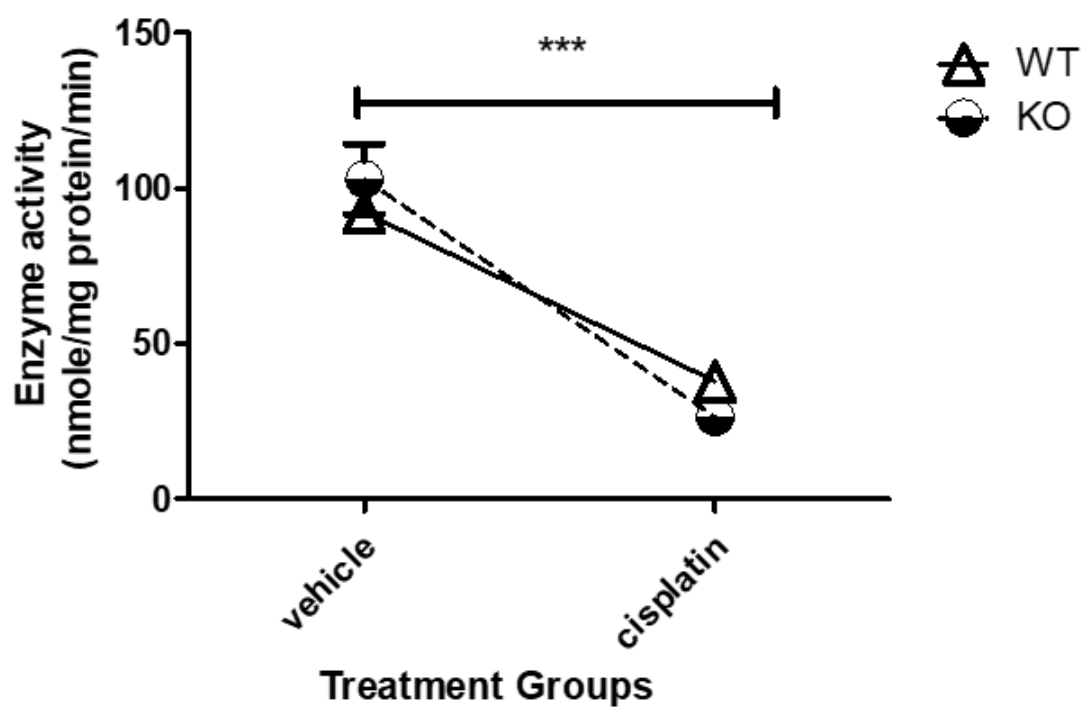

Figure 3.2: Effect of cisplatin treatment on glucose-6-phosphatase enzyme activity in WT and NHERF1 KO mouse kidneys.

2-4 month old male C57BL/6J WT and NHERF1 KO mice were given cisplatin (20 mg/kg dose IP) or vehicle (saline) and sacrificed after 72 hours as described in Chapter II. G6Pase enzyme activity was determined from kidney cortex tissue of these mice. Data are means \pm SEM (WT vehicle $n=3)$, (KO vehicle $n=4)$, (WT cisplatin $\mathrm{n}=3$ ), and $(\mathrm{KO}$ cisplatin $\mathrm{n}=5) .{ }^{* *} P<0.001$ cisplatin treated WT and NHERF1 KO mice compared to vehicle saline controls. 
significantly affect lactate dehydrogenase activity in these mouse kidneys [(WT vehicle: $0.06 \mathrm{nmole} / \mathrm{mg}$ protein $/ \mathrm{min} \pm 0.02)$, (KO vehicle: $0.1 \mathrm{nmole} / \mathrm{mg}$ protein $/ \mathrm{min} \pm 0.05)$, (WT cisplatin: $0.1 \mathrm{nmole} / \mathrm{mg}$ protein $/ \mathrm{min} \pm 0.8)$, and (KO cisplatin: $0.02 \mathrm{nmole} / \mathrm{mg}$ protein/min \pm 0.006$)$ ] (Figure 3.3).

\section{Cisplatin treatment significantly decreases malate dehydrogenase enzyme activity in both WT and NHERF1 KO mice.}

Malate dehydrogenase (MDH) is an enzyme involved in many metabolic pathways including the citric acid cycle. MDH reversibly catalyzes the oxidation of malate to oxaloacetate with the reduction of $\mathrm{NAD}^{+}$to $\mathrm{NADH}(124)$. The effect of NHERF1 loss and/or cisplatin treatment on MDH activity was analyzed. In a similar way to LDH, NHERF1 loss or cisplatin treatment did not significantly affect $\mathrm{MDH}$ activity in these mouse kidneys [(WT vehicle: $0.9 \mathrm{nmole} / \mathrm{mg}$ protein $/ \mathrm{min} \pm$ 0.06), (KO vehicle: $0.7 \mathrm{nmole} / \mathrm{mg}$ protein/min \pm 0.02 ), (WT cisplatin: 0.8 nmole $/ \mathrm{mg}$ protein $/ \mathrm{min} \pm 0.02$ ), and (KO cisplatin: $0.81 \mathrm{nmole} / \mathrm{mg}$ protein $/ \mathrm{min} \pm$ 0.06)] (Figure 3.4).

5. NHERF1 loss affects malic enzyme activity before and after cisplatin treatment.

Malic enzyme (ME) catalyzes the conversion of malic acid to pyruvate and produces NADPH (125). ME serves as an additional source of NADPH for lipogenesis. In order to understand the affect that NHERF1 loss and/or cisplatin 
Figure 3.3

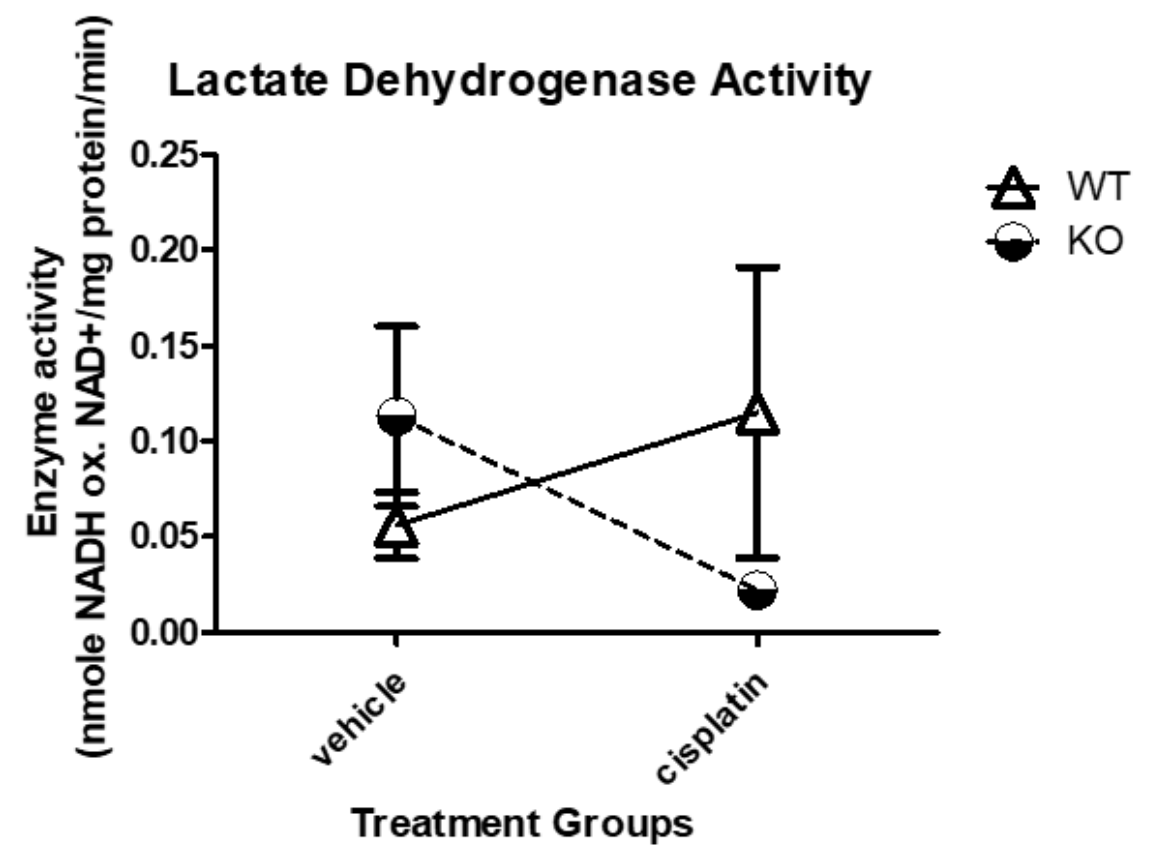

Figure 3.3: Lactate Dehydrogenase enzyme activity in WT and NHERF1 KO mouse kidneys.

2-4 month old male C57BL/6J WT and NHERF1 KO mice were given cisplatin (20 mg/kg dose IP) or vehicle (saline) and sacrificed after 72 hours as described in Chapter II. LDH enzyme activity was determined from kidney cortex tissue of these mice. Data are means \pm SEM (WT vehicle $n=3)$, (KO vehicle $n=4)$, (WT cisplatin $n=3$ ), and (NHERF1 KO cisplatin $n=5$ ). No significant differences were recorded. 
Figure 3.4

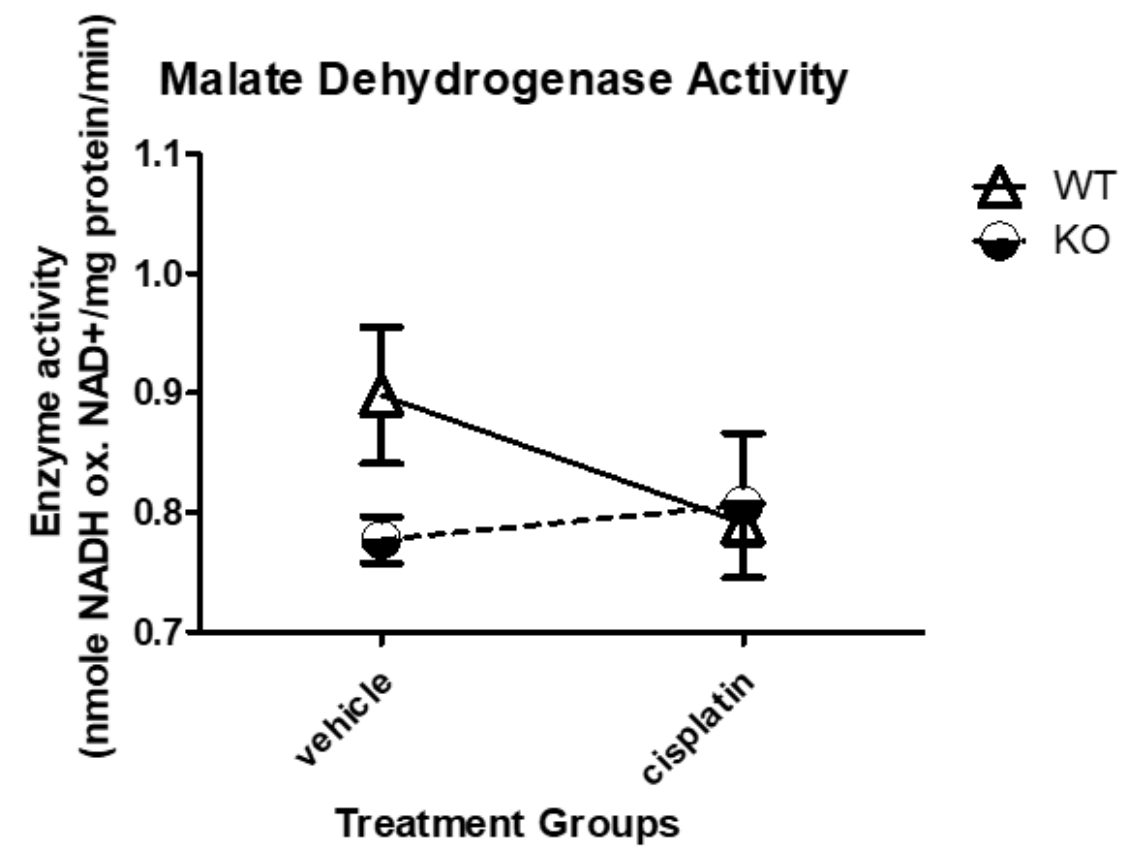

Figure 3.4: Malate Dehydrogenase enzyme activity in WT and NHERF1 KO mouse kidneys.

2-4 month old male C57BL/6J WT and NHERF1 KO mice were given cisplatin (20 mg/kg dose IP) or vehicle (saline) and sacrificed after 72 hours as described in Chapter II. MDH enzyme activity was determined from kidney cortex tissue of these mice. Data are means \pm SEM (WT vehicle $n=3)$, (KO vehicle $n=4)$, (WT cisplatin $n=3)$, (NHERF1 KO cisplatin $n=5)$. No significant differences were reported. 
treatment may have on ME activity, kidney cortex tissue from vehicle or cisplatin treated WT and NHERF1 KO were evaluated as described in Chapter II.

Interestingly, there was a significant genotype effect on ME activity resulting in an increase in activity in NHERF1 KO kidneys $(P=0.0065)$ (Figure 3.5).

Additionally, a significant interaction affect was also noted between WT and NHERF1 KO kidneys after cisplatin treatment $(P=0.0005)$ [(WT vehicle: 0.07 $\mathrm{nmole} / \mathrm{mg}$ protein $/ \mathrm{min} \pm 0.012)$, (KO vehicle: $0.21 \mathrm{nmole} / \mathrm{mg}$ protein $/ \mathrm{min} \pm 0.01)$, (WT cisplatin: $0.15 \mathrm{nmole} / \mathrm{mg}$ protein/min \pm 0.012 ), and (KO cisplatin: 0.13 nmole/mg protein/min \pm 0.02$)$ ] (Figure 3.5).

\section{NHERF1 loss affects glucose-6-phosphate dehydrogenase enzyme activity before and after cisplatin treatment.}

Cisplatin-induced $\mathrm{AKI}$ is known to decrease intermediates of the pentose phosphate pathway $(86,94)$ in mice. Glucose-6-phosphate dehydrogenase (G6PD) is a cytosolic enzyme that participates in the pentose phosphate pathway, resulting in NADPH production (126). This is accomplished when G6PD reduces NADP+ to NADPH while oxidizing glucose-6-phosphate (126). G6PD enzyme activity was analyzed in vehicle and cisplatin treated WT and NHERF1 KO kidney cortex to elucidate if NHERF1 loss and/or cisplatin treatment affected the pentose phosphate pathway. Similarly to ME, there was a significant genotype effect on G6PD activity resulting in an increase in activity in NHERF1 KO kidneys $(P=0.0033)$ (Figure 3.6). Additionally, a significant interaction affect was also noted between WT and NHERF1 KO kidneys after cisplatin treatment 


\section{Figure 3.5}

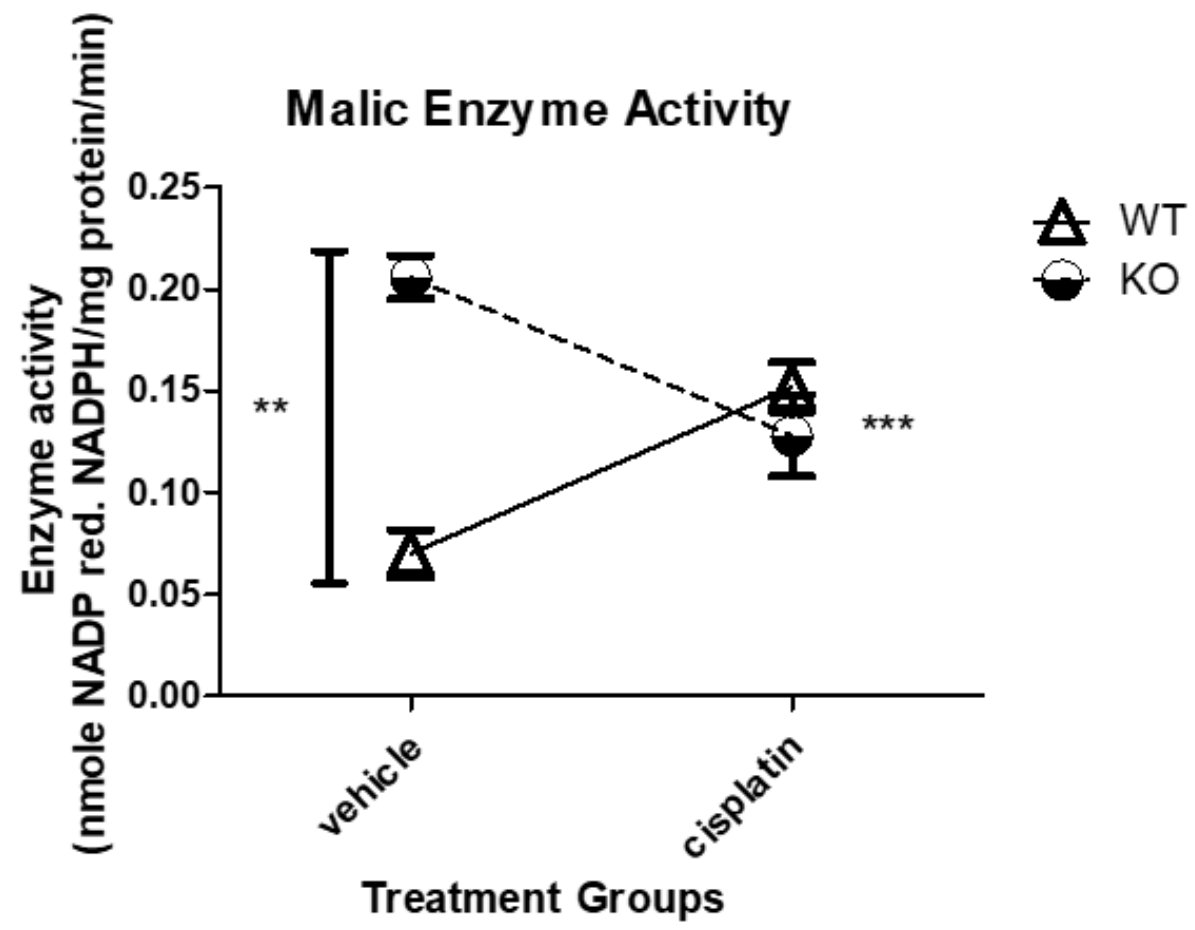

Figure 3.5: Effect of NHERF1 loss and cisplatin treatment on malic enzyme activity in WT and NHERF1 KO mouse kidneys.

2-4 month old male C57BL/6J WT and NHERF1 KO mice were given cisplatin (20 mg/kg dose IP) or vehicle (saline) and sacrificed after 72 hours as described in Chapter II. ME activity was determined from kidney cortex tissue of these mice. Data are means \pm SEM (WT vehicle $n=3)$, (KO vehicle $n=4)$, (WT cisplatin $\mathrm{n}=3$ ), and (KO cisplatin $\mathrm{n}=5) .{ }^{* *} P=0.0065$ vehicle treated NHERF1 KO mice compared to WT vehicle controls; ${ }^{* * *} P=0.0005$ interaction of cisplatin treated NHERF1 KO mice to cisplatin treated WT mice. 


\section{Figure 3.6}

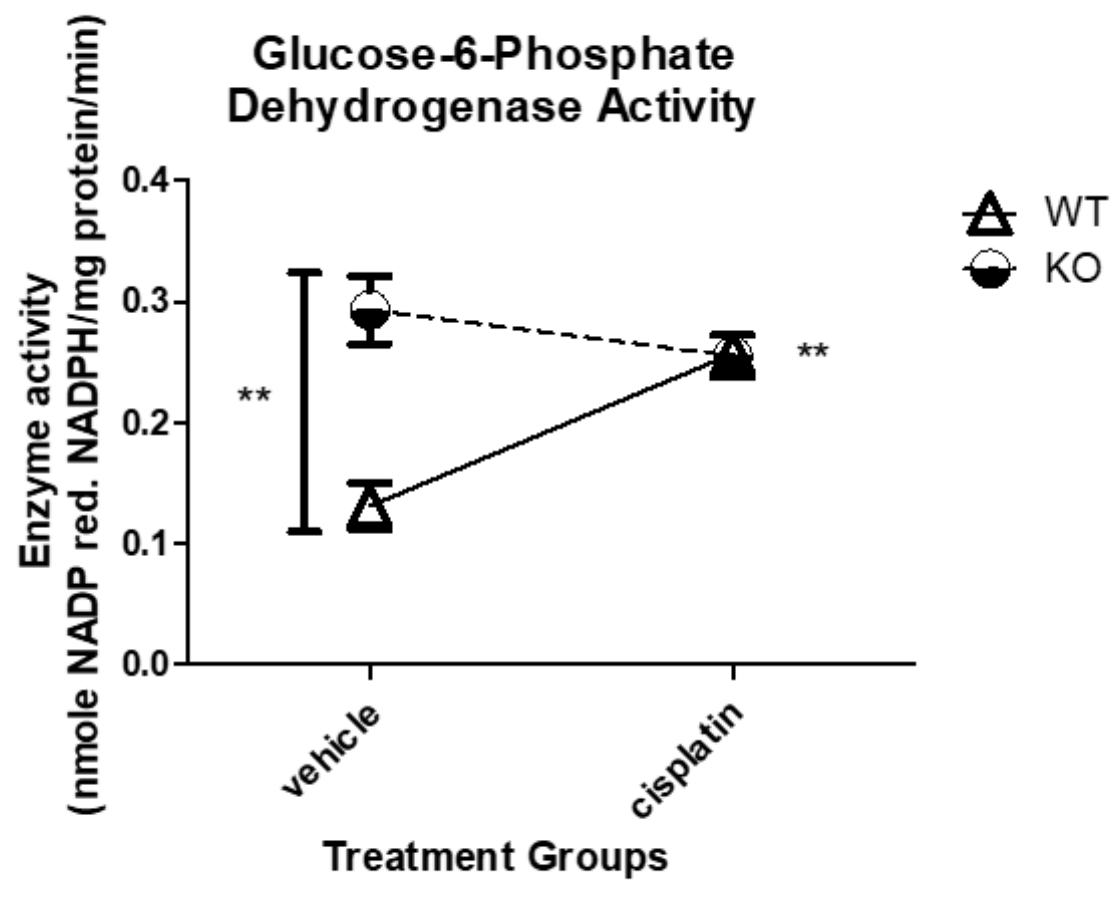

Figure 3.6: Effect of NHERF1 loss and cisplatin treatment on glucose-6-

phosphate dehydrogenase enzyme activity in WT and NHERF1 KO mouse kidneys.

2-4 month old male C57BL/6J WT and NHERF1 KO mice were given cisplatin (20 mg/kg dose IP) or vehicle (saline) and sacrificed after 72 hours as described in Chapter II. G6PD enzyme activity was determined from kidney cortex tissue of these mice. Data are means \pm SEM (WT vehicle $n=3)$, (KO vehicle $n=4)$, (WT cisplatin $\mathrm{n}=3$ ), and (KO cisplatin $\mathrm{n}=5) .{ }^{* *} P=0.0065$ vehicle treated NHERF1 KO mice compared to WT vehicle controls; ${ }^{* *} P=0.0005$ interaction of cisplatin treated NHERF1 KO mice to cisplatin treated WT mice. 
$(P=0.00029)$ [(WT vehicle: $0.13 \mathrm{nmole} / \mathrm{mg}$ protein $/ \mathrm{min} \pm 0.02),($ KO vehicle: 0.3 $\mathrm{nmole} / \mathrm{mg}$ protein/min \pm 0.03 ), (WT cisplatin: $0.3 \mathrm{nmole} / \mathrm{mg}$ protein $/ \mathrm{min} \pm 0.007$ ), and (KO cisplatin: $0.3 \mathrm{nmole} / \mathrm{mg}$ protein/min \pm 0.02 )] (Figure 3.6).

\section{NHERF1 loss does not affect amount of ATP by LC-MS in mouse}

\section{kidneys.}

ATP provides energy to drive many cellular processes and is consumed during many metabolic processes (127). In eukaryotes ATP is produced by three different metabolic pathways: [1] glycolysis, [2] the citric acid cycle or oxidative phosphorylation, and [3] beta-oxidation (127). In order to determine if NHERF1 KO kidneys had differences in ATP content, kidneys were snap-frozen and processed while cold for LC-MS as described in Chapter II. LC-MS analysis revealed that there were no significant differences in ATP amount in WT (3.4 nmoles $/ \mathrm{mg}$ tissue \pm 0.5$)$ and NHERF1 KO (3.1 nmoles $/ \mathrm{mg}$ tissue \pm 0.5$)$ kidneys $(P=0.67)$ (Figure 3.7).

\section{Discussion}

This work had two primary goals: [1] to characterize any metabolic differences in NHERF1 KO mouse kidneys and [2] to evaluate if metabolic changes in NHERF1 KO mouse kidneys could predispose these animals to susceptibility of cisplatin-induced AKI. As discussed in the introduction, previous studies noted metabolic changes in affected mouse kidneys post cisplatin treatment, contributing to both injury and the development of CKD. These studies revealed 
Figure 3.7

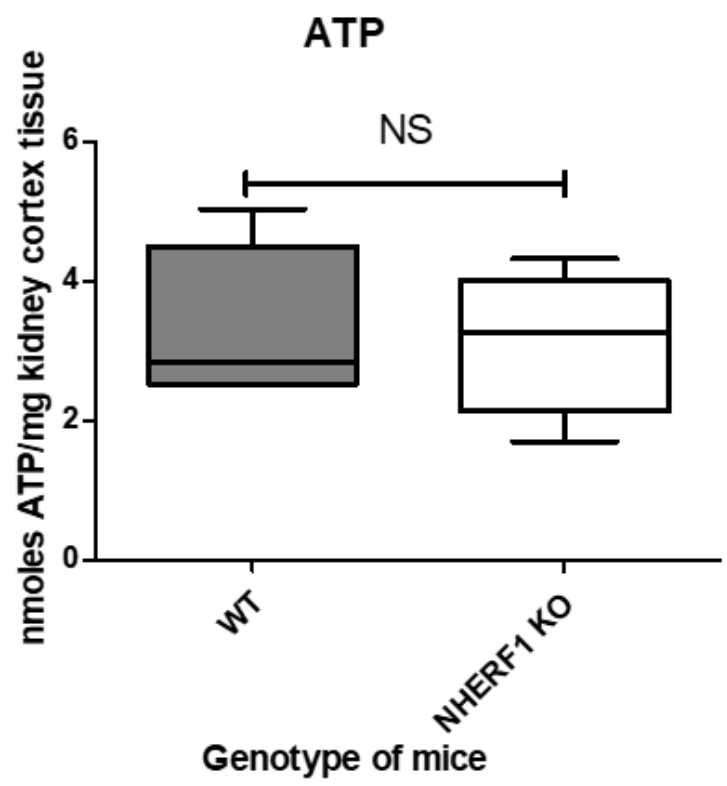

Figure 3.7: ATP content of WT and NHERF1 KO mouse kidneys.

2-4 month old male C57BL/6J WT and NHERF1 KO mice kidneys were removed and snap-frozen in liquid nitrogen. LC-MS was utilized to evaluate amount of ATP in these tissues as described in Chapter II. Data are means \pm SEM (WT $n=5)$ and $(K O n=5)$. No significant differences were reported in these kidneys. 
that cisplatin nephrotoxicity depletes amino acids (86-89), reduces fatty acid oxidation $(86,89,91)$, reduces glycolytic enzymes $(86,93)$, and decreases intermediates of the pentose phosphate pathway and the citric acid cycle (86, 94).

One of the first objectives of this study was to evaluate any changes in gluconeogenesis of NHERF1 KO mouse kidneys compared to WT mouse kidneys pre and post cisplatin treatment. Baseline measurements of FBPase showed no changes in enzymatic activity with NHERF1 loss; furthermore, WT and NHERF1 KO kidneys both had a decrease in activity following cisplatin treatment. Indicating that FBPase functions similarly with or without NHERF1, consistent with other reports $(128,129)$. Likewise, G6Pase activity was unchanged with NHERF1 loss and, similarly to other reports, decreased after cisplatin treatment in both genotypes $(128,129)$. These data confirm that NHERF1 loss does not have an apparent effect on gluconeogenesis, whereas cisplatin injury results in decreased gluconeogenic enzymes. MDH and LDH belong to the same family of NAD-dependent enzymes, and cisplatin-induced oxidative stress has been shown to increase LDH due to lipid peroxidation and decrease $\mathrm{MDH}(130,131)$. Interestingly, this study found no significant changes in $\mathrm{LDH}$ and $\mathrm{MDH}$ activity regardless of genotype or cisplatin treatment, which is consistent with a previous study that demonstrated similar results where LDH and MDH activities were unchanged after cisplatin treatment in rats (132). These disparities between studies and cisplatin's effect on LDH and MDH activities further illustrate the complexity of this disease. 
ME and G6PD activity provide necessary NADPH, a key cofactor in redox control and reductive biosynthesis. ME plays a role in production of pyruvate and serves as an additional source of NADPH for lipogenesis. Mammals that utilize glucose for lipogenesis via citrate are believed to rely heavily on transferring reducing equivalents from extra-mitochondrial NADH to NADP through the combined acts of MDH and ME (134). Pyruvate from ME is available to re-enter the mitochondria and be converted to of oxaloacetate or acetyl-CoA (134). Additionally, there is recent evidence for direct cross-talk between ME and the pentose phosphate pathway (135), where G6PD is a rate limiting enzyme. Another goal of this study was to determine the effect of NHERF1 loss and/or the combined effect with cisplatin on ME and G6PD activity. Notably, both ME and G6PD activity were significantly increased in the NHERF1 KO mouse kidney under baseline conditions. Following cisplatin treatment ME and G6PD activity decreased until level with cisplatin treated WT activity and resulted in a significant interaction. These increases in ME and G6PD activity at baseline suggest some altered or compensatory metabolic changes in the pentose phosphate pathway of NHERF1 KO mice. One study found using a cell culture model of diabetes that increased activity of G6PD restored redox balance in endothelial cells exposed to high glucose levels (136), where high glucose levels had previously decreased G6PD and increased levels of oxidative stress. Multiple studies have noted the importance of cellular redox balance in both development of and in protection from renal injury $(103,104)$. In rats G6PD activity is reported to decrease in acute models of nephrotoxicity, and ME activity 
is reported to increase (129). Additionally, one other investigator found that NHERF1 is a previously unidentified regulator of Nox1 (NADPH oxidase) and further promotes Nox1 activity (81). In fact, vascular smooth muscle cells deficient of NHERF1 were unable to mount a ROS response to potent $\mathrm{O}_{2}$ stimuli (81). Taken together, one possible explanation for these data are that NHERF1 $\mathrm{KO}$ mice are under some stress resulting in an increase in G6PD activity in an attempt to restore redox balance. Whether, redox balance is indeed restored in these mice will be further investigated in Chapter V. These data suggest subtle metabolic changes in the kidneys of the NHERF1 KO animals, but do not allow us to make definitive conclusions on the mechanism involved. This is in part due to the amelioration of cisplatin nephrotoxicity when antioxidants are given or when there is a decrease in ROS prior to treatment. On the other hand, cisplatin does decrease intermediates of the pentose phosphate pathway. Indicating this could be a mechanism of enhanced susceptibility if the NHERF1 KO kidneys are more reliant on this pathway for energy generation.

Lastly, this study evaluated ATP levels between WT and NHERF1 KO kidneys to determine any alterations in cellular energy. Following LC-MS assessment it was determined that WT and NHERF1 KO kidneys contain similar levels of ATP, indicating that NHERF1 KO mice are not depleted and/or producing higher amounts of ATP due to energy demands. Collectively, the results of the ATP, G6PD and ME data point towards potential changes in NADPH of NHERF1 KO mice. This presents another area of interest to investigate in the future. 
In conclusion, this study provides insight into metabolic changes of NHERF1 KO mice. In this study increased enzymes of the pentose phosphate pathway were found in NHERF1 KO mice and suggest these animals are expressing some differences in metabolic pathways when compared to WT animals. The basis for these changes in the activity of this metabolic pathway and its significance for the increased susceptibility of NHERF1 KO mice to cisplatin nephrotoxicity remain unknown. These results provide another avenue to be explored in the future pertaining to NADPH levels in NHERF1 KO mouse kidneys. Further investigation into bioenergetics of the NHERF1 KO mouse kidneys may elucidate more insight into susceptibility to cisplatin injury. 


\section{CHAPTER IV}

\section{MECHANISM OF SUSCEPTIBILITY OF NHERF1 KO MICE TO CISPLATIN- INDUCED AKI IS NOT MITOCHODNRIAL DYSFUNCTION}

\section{A. Introduction}

Mitochondria are important for multiple cellular functions but are more commonly known as the 'powerhouse of the cell'. In all eukaryotic cells that do not undergo photosynthesis the mitochondria is the main source for ATP, an energy rich compound that drives cellular functions $(137,138)$. The inner mitochondrial membrane is the site of the ETC and oxidative phosphorylation where ATP is generated from ADP and phosphate ions $(137,138)$. Other mitochondrial functions apart from cellular respiration and ATP synthesis include: production of NADH and GTP in the citric acid cycle, amino acid biosynthesis, calcium signaling, stress responses, and cellular signaling $(137,138)$. On the other hand, when mitochondria are damaged they undergo electron leakage and produce massive amounts of free radicals in the form of ROS, leading to cell injury and death. Thus, mitochondrial dysfunction is often the cause of severe diseases.

Not surprisingly, the critical role of mitochondria in human health and disease progression has strengthened interest in the relationship of mitochondrial 
function and cisplatin nephrotoxicity. As previously discussed in Chapters I and III, cisplatin is a highly successful chemotherapeutic with nephrotoxic side effects. This toxicity greatly limits cisplatin's therapeutic use and often leads to the development of AKI (8). Pathologically cisplatin induces cell death and injury to the renal tubules, triggers vascular dysfunction, and launches a strong inflammatory response (8). These effects of cisplatin result in both necrosis and apoptosis in the proximal and distal tubules. Cisplatin's mechanism of toxicity remains uncertain to this day, however, cisplatin induced-mitochondrial dysfunction and the development of renal tubule cell damage/death provides an attractive hypothesis.

Cisplatin nephrotoxicity results in a decline in both state 3 mitochondrial respiration and calcium accumulation (139-141). Additionally, cisplatin nephrotoxicity has been associated with a decrease in cytochrome c oxidase activity and a reduction in complex IV protein expression (139-142). These changes can lead to increased ROS production from mitochondria. Moreover, cisplatin toxicity drastically reduces renal cell antioxidant activity $(139,140,142$, 143). Some studies found that cisplatin-induced mitochondrial damage results in a decrease in mitochondrial mass, disruption of cristae, and even mitochondrial swelling $(105,139,140)$. These morphological changes are associated with a significant reduction in mitochondrial activity and ATP production $(139,140)$. Furthermore, protein-bound platinum was found in renal tubule cell mitochondria 24 hours after cisplatin treatment (144). Together, these studies indicate a critical role of mitochondrial function and oxidative stress in cisplatin nephrotoxicity. 
These studies provided a new avenue of research to explore in understanding how NHERF1 loss increases susceptibility to cisplatin-induced AKI. In Chapter III NHERF1 loss was associated with an increase in ME and G6PD activity under baseline conditions and a differential response when compared to WT animals, suggesting compensatory mechanisms that may affect mitochondrial function. Previous studies from our laboratory comparing WT and NHERF1 deficient opossum kidney (OK) cells, a model of mammalian renal proximal tubule, demonstrated that the NHERF1 deficient cells grew more slowly and were more likely to die in culture with no apparent morphological differences. Additionally, proteomic studies revealed that NHERF1 KO mice exhibited markedly diminished associated mitochondrial proteins in the BBM of these mice (unpublished data). These properties of the NHERF1 deficient cells and mice (the poor survival, decreased expression of mitochondrial proteins in BBM, and aberrant pentose phosphate enzyme activity) suggested that NHERF1 loss may be associated with mitochondrial dysfunction and susceptibility to kidney injury. Based on these observations the goals of this work are twofold: [1] to determine if NHERF1 KO mice have altered mitochondrial function and/or structure and [2] to determine if NHERF1 KO mice have an underlying mitochondrial dysfunction that predisposes these animals to cisplatin nephrotoxicity.

\section{B. Experimental Procedures}

\section{Electron microscopy}


2-4 month old male WT and NHERF1 KO mice were perfused with $3 \%$ glutaraldehyde and the kidneys were removed for EM analysis as described in Chapter II.

\section{Seahorse XF24 mitochondrial respiration analysis}

Mitochondria were isolated from 2-4 month old male WT and NHERF1 KO mouse kidney homogenates. Seahorse XF24 analysis of OCR of isolated mitochondria were obtained as described in Chapter II.

\section{Data analysis}

EM and Seahorse XF24 mitochondrial data were analyzed as described in Chapter II.

\section{Statistical analysis}

Statistical analysis of electron microscopy and Seahorse XF24 mitochondrial data was performed as described in Chapter II.

\section{Results}

\section{NHERF1 loss does not affect kidney proximal tubule mitochondria morphology.}

The mitochondrial structure is essential for proper function, thus, EM images of WT and NHERF1 KO proximal tubule mitochondria were utilized to evaluate their morphology as described in Chapter II. These images were taken and evaluated by a renal pathologist for signs of injury, oxidative stress, and changes 
in cristae. There were no apparent changes in mitochondria morphology between WT and NHERF1 KO proximal tubules (Figure 4.1). The only injury reported was early ischemic changes mostly likely due to harvesting of the kidneys (Figure 4.1). Some endosomal swelling was noted but occurred across both genotypes. Additionally, in figure 4.1 the density and distribution of mitochondria within the tubules were also alike. Lastly, no apparent signs of oxidative stress were found in either genotype (Figure 4.1).

\section{NHERF1 loss does not affect mitochondria number or area in kidney proximal tubules.}

Changes in mitochondrial number and a decrease in size have been associated with a decline in mitochondrial function (145). Therefore, one goal of this study was to determine if mitochondrial number and/or size changed in NHERF1 KO proximal tubules when compared to WT. Images from EM of WT and NHERF1 KO kidney proximal tubules were utilized in order to count the number of mitochondria and to calculate the average area via Image J. There was not a significant difference between the average number of mitochondria between WT and NHERF1 KO tubules (WT average number: 128.8) and (NHERF1 KO average number: 115) $(P=0.6)$ (Figure 4.2A). In addition, there was not a significant difference between the average area of mitochondria in WT and NHERF1 KO tubules (WT average area: 580,540.9 $\mu^{2}$ ) and (NHERF1 KO average area: $\left.678,465.4 \mu \mathrm{m}^{2}\right)(P=0.75)$ (Figure 4.2B). 


\section{Figure 4.1}
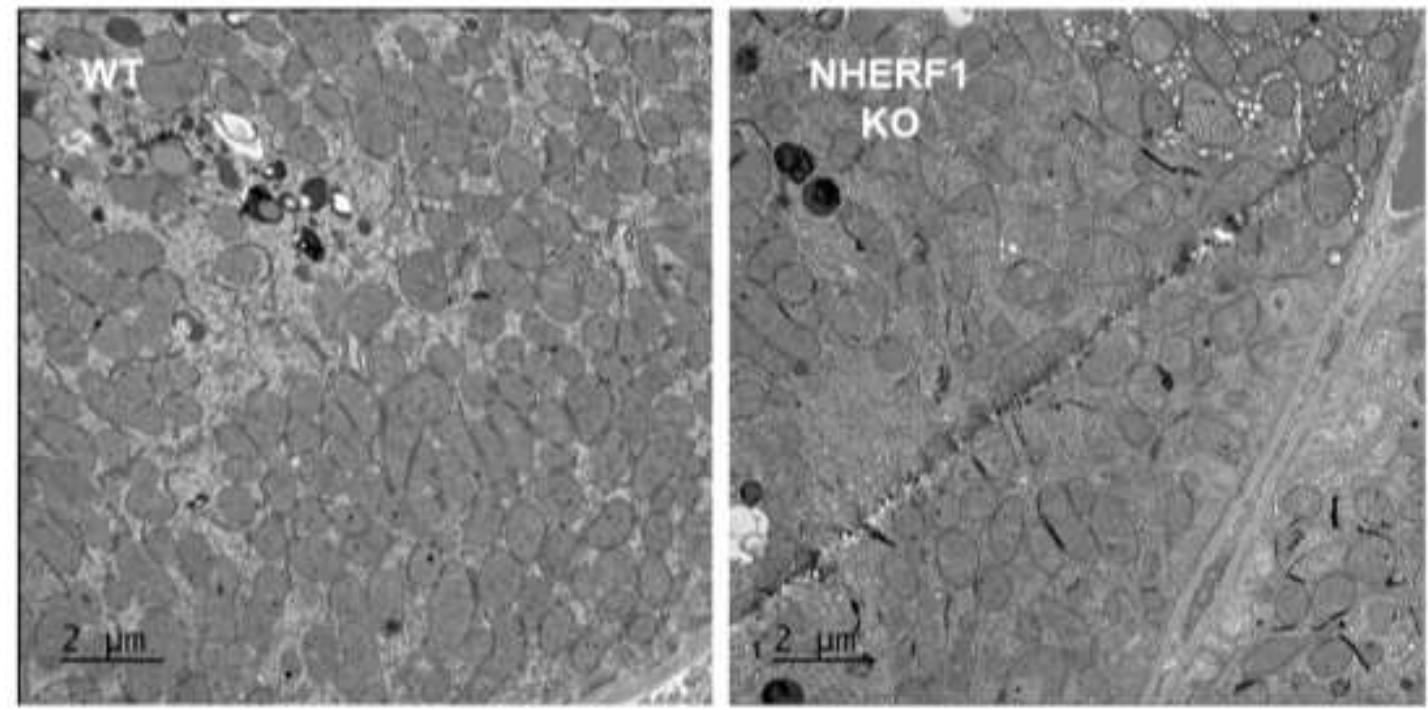

Figure 4.1: Electron microscopy of WT and NHERF1 KO proximal tubule mitochondria.

Representative photomicrographs of a $4 \mathrm{x}$ field of WT and NHERF1 KO proximal tubule mitochondria. The left panel represents proximal tubule mitochondria of WT mice, while the right panel represents NHERF1 KO proximal tubule mitochondria (WT $n=6$ ) and (NHERF1 KO $n=5)$. The scale bars were set at $2 \mu \mathrm{m}$. 


\section{Figure 4.2}

A

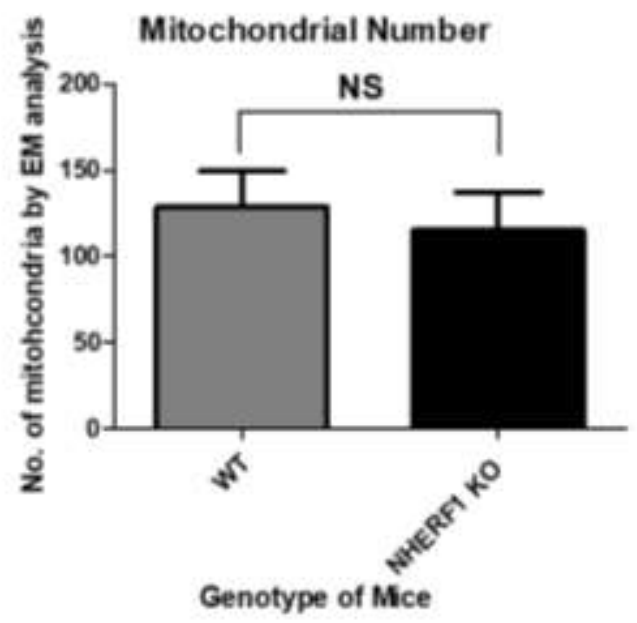

B

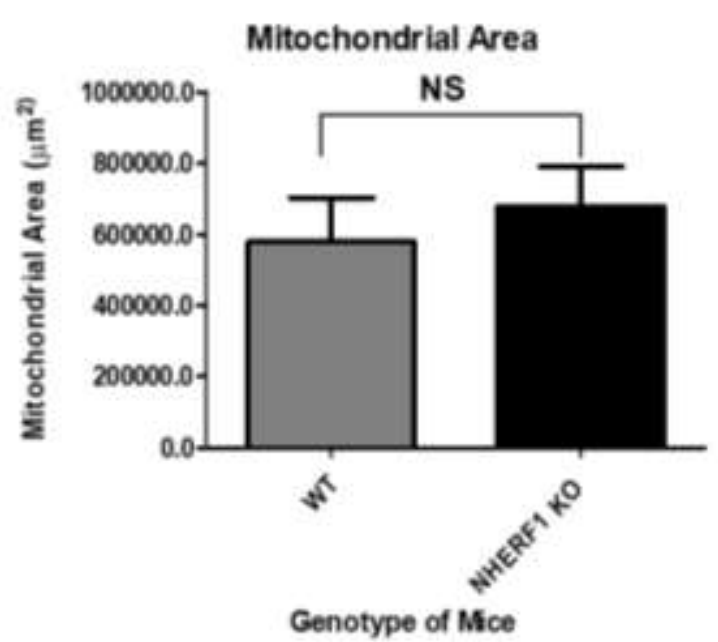

Figure 4.2: Mitochondrial number and area of WT and NHERF1 KO mouse proximal tubules.

2-4 month old male WT and NHERF1 KO mice were perfused with 3\% glutaraldehyde for EM analysis. (A) Number of mitochondria were counted in random $4 x$ visual fields with the highest density of mitochondria. Data are means \pm SEM (WT $n=6)$ and (NHERF1 KO $n=5)$. Mitochondria number of NHERF1 KO proximal tubules were insignificant when compared to WT. (B) Mitochondria area was calculated using EM images and Image J. Data are means \pm SEM (WT $n=6$ ) and (NHERF1 KO $\mathrm{n}=5$ ). Mitochondria area of NHERF1 KO proximal tubules were insignificant when compared to WT. 


\section{WT and NHERF1 KO mouse kidney mitochondria have similar oxidative capacities.}

The mitochondria's capacity to reduce oxygen is a critical aspect in the process of mitochondrial electron transport and ATP synthesis. Therefore, measuring mitochondrial oxygen consumption can provide a valuable method to assess mitochondrial function. One purpose of this work was to assess mitochondrial function by oxidative capacity in WT and NHERF1 KO kidneys using the Seahorse XF24 analyzer. In panel A of figure 4.3 OCR of WT and NHERF1 KO kidney mitochondria are shown over time. Both WT and NHERF1 $\mathrm{KO}$ mitochondria exhibit a similar trend and response to added substrates and inhibitors. When adding the substrate Succinate/Rotenone plus ADP for production of ATP both genotypes exhibit a maximal increase in OCR. Moreover, both genotypes undergo a decrease in OCR after adding Oligomycin, an inhibitor of Complex V (formation of ATP from ADP via $\mathrm{O}_{2}$ consumption). Lastly, Antimycin A shuts down all respiration, where the OCR is close to the basal OCR. The difference between the basal OCR and OCR after Antimycin A is the non-mitochondrial respiration.

Changes in state 3 (conversion of ATP from ADP and consumption of $\mathrm{O}_{2}$ ) and state 4 (non-phosphorylating or resting respiration) respiration are commonly used to evaluate mitochondria oxidative capacity. Panel B of figure 4.3 shows both state 3 [(WT: $60 \mathrm{pmoles} / \mathrm{min} / \mu \mathrm{g}$ protein \pm 6 ) and (NHERF1 KO: 44 
Figure 4.3

A

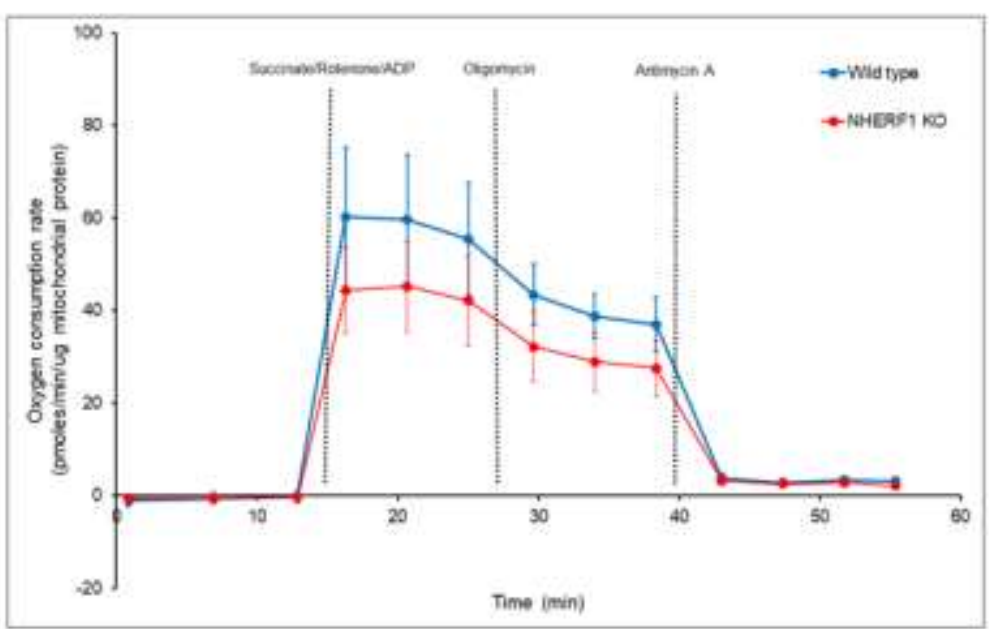

B

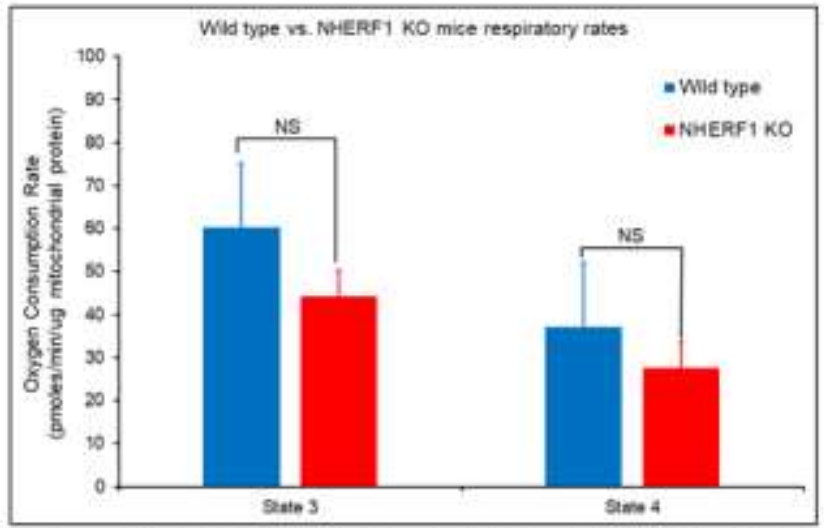

State 3; phosphorylating respiration in the presence of substrate + ADP (comversion of ADP to ATP consumes $\mathrm{O}_{2}$ ) State 4: non-phosphorylating or resting respiration

C

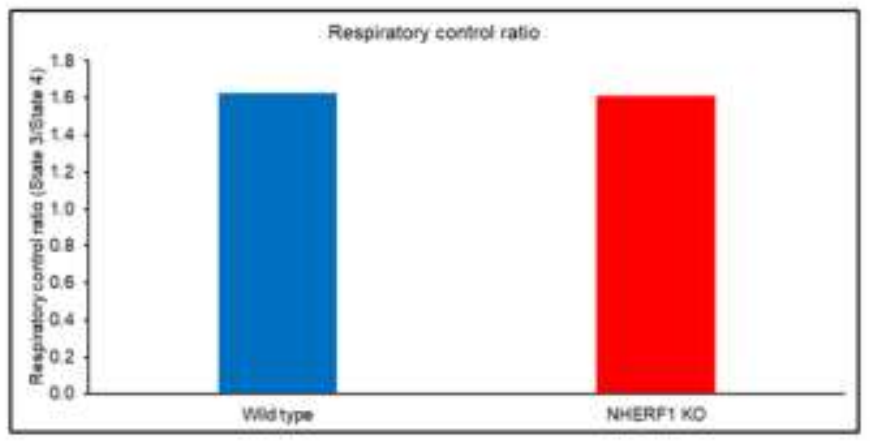


Figure 4.3: Mitochondrial function in isolated mitochondria of WT and NHERF1 KO kidneys.

Mitochondria from 2-4 month old male WT and NHERF1 KO mice were isolated and analyzed via the Seahorse XF24 for oxidative capacity as described in Chapter II. (A) OCR was recorded after the addition of both substrates (Succinate/Rotenone/ADP) and inhibitors (Oligomycin and Antimycin A) [(WT $\mathrm{n}=6$ ) and (NHERF1 KO $\mathrm{n}=6)$ ]. (B) State 3 and State 4 was calculated using the recorded OCR's of WT and NHERF1 KO mitochondria. Data are means \pm SEM [(WT $\mathrm{n}=6)$ and (NHERF1 KO n=6)]. State 3 and State 4 respiration were considered insignificant between WT and NHERF1 KO mouse kidney mitochondria. (C) Respiratory control ratio (RCR) (state 3/state 4) was calculated between WT and NHERF1 KO kidney mitochondria. Data are represented as state $3 /$ state 4 ratio [(WT $n=6)$ and $(N H E R F 1 n=6)]$. RCR was insignificant between WT and NHERF1 KO mouse kidney mitochondria. 
$\mathrm{pmoles} / \mathrm{min} / \mu \mathrm{g}$ protein \pm 2$)]$ and 4 [(WT: $37 \mathrm{pmoles} / \mathrm{min} / \mu \mathrm{pg}$ protein \pm 6$)$ and (NHERF1 KO: 28 pmoles/min/ $\mu$ g protein \pm 2 )] of WT and NHERF1 KO kidney mitochondria, where state $3(P=0.2)$ and $4(P=0.1)$ were determined to be insignificant between the groups.

The respiratory control ratio $(\mathrm{RCR})$ is the best general measure of mitochondrial function in isolated mitochondria. RCR is measured by taking state $3 /$ state 4 respiration and sums up the main function of mitochondria: the ability to idle at low rates and then respond to ADP by making ATP at high rates. In addition the RCR has no absolute value that is diagnostic of mitochondrial dysfunction (146). Thus, RCR values are substrate and tissue dependent, making this complex function advantageous when measuring mitochondrial function in isolated mitochondria (146). A change in almost any aspect of oxidative phosphorylation will result in a change in the RCR when comparing isolated mitochondria (146). Accordingly, the RCR was calculated between WT (1.63) and NHERF1 (1.61) KO kidney mitochondria and also found to be insignificant (Figure 4.3C).

\section{Discussion}

The goal of this study was to determine if NHERF1 loss results in mitochondrial dysfunction. This possibility was investigated as a potential mechanism of susceptibility of NHERF1 KO mice to cisplatin-induced AKI. This is the first study to investigate mitochondrial dysfunction in NHERF1 KO mice, and 
the first to show that NHERF1 KO mice do not have an underlying mitochondrial dysfunction.

The morphology of proximal tubule mitochondria of WT and NHERF1 KO mice was determined to be similar. Structural changes in mitochondria are indicative of improper function thus; these observations indicate that NHERF1 KO mice proximal tubule mitochondria do not have structural changes leading to dysfunction. In addition to structural changes, mitochondrial number and size were also evaluated. With age, mitochondrial integrity and functionality are known to decrease due to mutations and oxidative damage by ROS (145). Moreover, in aged mice (22-28 months), mitochondria exhibit lowered oxidative capacity, reduced oxidative phosphorylation, decreased ATP production, significant increase in ROS generation, and diminished antioxidant defense (145, 147). In addition, with age mitochondrial biogenesis declines due to alterations in mitochondrial dynamics and inhibition of mitophagy, an autophagy process that removes dysfunctional mitochondria. Thus, one question postulated was if NHERF1 KO mice had a change in mitochondrial number and/or area that would indicate inadequate functioning or stress. EM analysis found that both mitochondrial number and area were similar between both genotypes, suggesting proper mitochondrial integrity and biogenesis in NHERF1 KO mice proximal tubules.

Assessing mitochondrial dysfunction requires a definition of that dysfunction, usually defined as the mitochondria's capability of making ATP in response to energy demands. Furthermore, this dysfunction can be evaluated in isolated 
mitochondria. For isolated mitochondria the best method of evaluating dysfunction is analyzing mitochondrial oxidative capacity and respiratory control. Respiratory control is the increase in respiration in response to ADP. The RCR is both substrate and tissue dependent, however, when comparing different study groups a high RCR value indicates good mitochondrial function, while a low RCR indicates mitochondrial dysfunction. When comparing WT and NHERF1 KO mitochondrial function state 3 and state 4 were determined to be similar between the genotypes. Furthermore, the RCR (state 3/state 4) was determined to be the same (NHERF1 KO: 1.61 vs WT: 1.63). These data indicate that NHERF1 KO mouse kidney mitochondria are responding accordingly to energy demands when isolated from tissue. However, isolated mitochondria removed from their natural environment and the effect of the intracellular milieu on mitochondria function cannot be assessed in this type of assay. Previous studies established that NHERF1 KO mice exhibited phosphate wasting (148). This phenotype could create an environment where mitochondria cannot function properly. When isolating mitochondria both the isolation buffer and respiration buffer contain phosphate to ensure the isolation process does not harm the mitochondria's ability to function. Therefore, a future project would be to evaluate mitochondrial function using whole kidney tissue with the Seahorse XF24 analyzer. This experiment could help clarify if NHERF1 KO mouse kidneys have an underlying mitochondrial dysfunction due to their environment and hence serving to answer the question if an underlying mitochondrial dysfunction could predispose NHERF1 KO mice to cisplatin nephrotoxicity. 
This study demonstrates that proximal tubule mitochondria from NHERF1 KO kidneys are structurally similar and healthy when compared to WT mitochondria. Additionally, isolated mitochondria from NHERF1 KO mice do not exhibit changes in function indicative of stressed or dysfunctional kidney mitochondria. These data do not support the hypothesis that NHERF1 loss results in an underlying mitochondrial dysfunction. However, one potential mechanism that has yet to be investigated is whether NHERF1 loss and the subsequent low phosphate environment affects kidney mitochondrial function. This information may provide an alternative mechanism of susceptibility to cisplatin nephrotoxicity in NHERF1 KO mice, and it will be investigated in the future. Understanding the underlying mechanism of susceptibility to cisplatin-induced AKI with NHERF1 loss may provide future therapeutic targets and or biomarkers to use in the clinic. 


\section{CHAPTER V}

\section{NHERF1 LOSS AFFECTS GGT ACTIVITY IN RESPONSE TO CISPLATIN}

\section{A. Introduction}

Cisplatin is one of the most effective chemotherapeutic agents used to treat a number of diseases including: head and neck, esophageal, bladder, testicular, ovarian, uterine, cervical, breast, stomach, small cell, and non-small cell lung cancer (8-10). As a chemotherapeutic cisplatin crosslinks with purine bases in DNA and inhibits DNA synthesis (9). Because cisplatin shows its highest activity in highly proliferative cells, its unusual dose-dependent and cumulative side effect of nephrotoxicity remains a mystery (8-10). In addition, some patients appear to have an increased sensitivity to this side effect, with $20-30 \%$ developing cisplatin-induced AKI with a single dose (8). This sensitivity remains an important area of research as there are no FDA-approved strategies or approved therapies for preventing or ameliorating cisplatin-induced AKI.

In hopes of tackling this urgent clinical need, much research has been driven in understanding how cisplatin is handled by the kidneys. To date the accepted hypothesis for the renal handling of cisplatin is that during glomerular filtration cisplatin accumulates in the kidneys (149). OCT2 and Ctr1 have been identified 
as membrane transporters capable of cisplatin uptake in renal proximal tubule cells (150). Furthermore, MATE1 has also been identified in transporting cisplatin from the proximal tubule to the urine (22). Cisplatin nephrotoxicity was ameliorated with OCT2 inhibition and is exacerbated with MATE1 inhibition in mice $(18,22,150)$. Biotransformation of cisplatin is believed to start immediately after transportation into the apical renal epithelial cell (25). The initial biotransformation step is proposed to be with the formation of glutathione conjugates (25). These conjugates are then cleaved to cysteinyl-glycineconjugates by GGT, which is expressed on the surface of proximal tubule cells (25). From there AP is thought to convert cysteinyl-glycine-conjugates to cysteine conjugates, which are then believed to be further metabolized by CCBL into highly reactive nephrotoxic thiols (25). With that series in mind, much of the biotransformation of cisplatin in the kidney remains controversial. For example, it is known that GGT has the highest activity in the kidney and it was thus considered a potential target for attenuation of nephrotoxicity. However, investigation into that possibility yields controversial results, suggesting that enhanced GGT activity may either increase or decrease sensitivity to cisplatininduced AKI (151).

These conflicting results have made the development of reno-protective strategies or therapies difficult. Recently, a new potential target, NHERF1, a known scaffolding protein important to the proper function of renal proximal tubule cells, has been identified. In this recent study NHERF1 KO mice exhibited exacerbated cisplatin nephrotoxicity, manifested by a significant increase in BUN, 
NGAL, and higher histologic injury severity scores than in cisplatin treated WT mice (35). Interestingly, NHERF1 KO mice showed similar levels of apoptosis when compared to WT mice, but had a clear increase in necrotic cell death following cisplatin treatment (35). Other studies noted that cisplatin nephrotoxicity was dose dependent and involved both necrosis and apoptosis, however, higher levels of necrosis were associated with higher concentrations of cisplatin (152, 153). Collectively, these data along with another study showing NHERF1 expression was also involved with cisplatin resistance in cervical cancer cells (110), suggested that loss of NHERF1 may affect the renal handling of cisplatin.

The goal of this work was to elucidate new potential mechanisms of susceptibility to cisplatin-induced AKI. NHERF1 loss was hypothesized to affect the renal handling of cisplatin by alterations in renal uptake and/or metabolism. In this endeavor the current understanding of cisplatin's uptake and biotransformation was utilized to evaluate NHERF1's effect on cisplatin-induced AKI.

\section{B. Experimental Procedures}

\section{Animals and treatments}

Male (2-4 month) WT and NHERF1 KO mice were treated with cisplatin (20 $\mathrm{mg} / \mathrm{kg} \mathrm{IP}$ ) for 4,24 , or 72 hours as described in Chapter II.

\section{2. y-Glutamyl Transferase activity assay}

The GGT activity assay was performed as described in Chapter II. 


\section{Histology and immunohistochemistry}

$\mathrm{H} \& \mathrm{E}, \mathrm{PAS}$, and IHC were performed as described in Chapter II.

\section{NGAL}

NGAL was determined as described in Chapter II.

\section{TBARS}

TBARS assay was performed as described in Chapter II.

\section{HPLC}

Blood and tissue samples for GSH, GSSG, Cys, CySS were processed and analyzed as described in Chapter II.

\section{Immunoblots}

Western blots were performed as described in Chapter II.

\section{Data analysis}

Data analysis was performed as described in Chapter II.

\section{Statistical analysis}

Statistical analysis was performed as described in Chapter II.

\section{Results}




\section{NHERF1 loss results in early cisplatin-induced renal histologic injury.}

H\&E and PAS staining of the kidneys of cisplatin treated mice after 24 hours revealed that NHERF1 KO mice exhibited early histologic injury that does not correspond with treated WT mice at the same time point (Figure 5.1). Similarly to the 72 hour time point, the injury is predominantly cortical in NHERF1 KO mice at 24 hours. This injury was manifested by intermittent casts, atrophy, and BBM sloughing (Figure 5.1) in NHERF1 KO mice, while cisplatin treated WT mice exhibited no obvious signs of histologic damage.

\section{Cisplatin treatment significantly increases urine NGAL protein in} NHERF1 KO mice in comparison to cisplatin treated WT mice at 24 hours.

In order to confirm cisplatin treatment resulted in early higher kidney injury in NHERF1 KO mice urine NGAL was measured as described in Chapter II. With cisplatin treatment both WT $(0.1 \mu \mathrm{g} / \mathrm{mL} \pm 0.02)$ and NHERF1 $\mathrm{KO}(0.7 \mu \mathrm{g} / \mathrm{mL} \pm$ 0.2) mice had a significant increase in NGAL protein compared to vehicle treated WT $(0.04 \mu \mathrm{g} / \mathrm{mL} \pm 0.03)$ and NHERF1 KO $(0.02 \mu \mathrm{g} / \mathrm{mL} \pm 0.006)(P=0.007)$ (Figure 5.2). However, a statistically significant increase in NGAL urine protein level was observed in cisplatin treated NHERF1 KO compared to cisplatin treated WT mice $(P=0.03)$ (Figure 5.2). Additionally, a significant decrease in NGAL urine protein in vehicle treated NHERF1 KO compared to vehicle treated WT mice was found $(P=0.04)$ (Figure 5.2). 


\section{Figure 5.1}
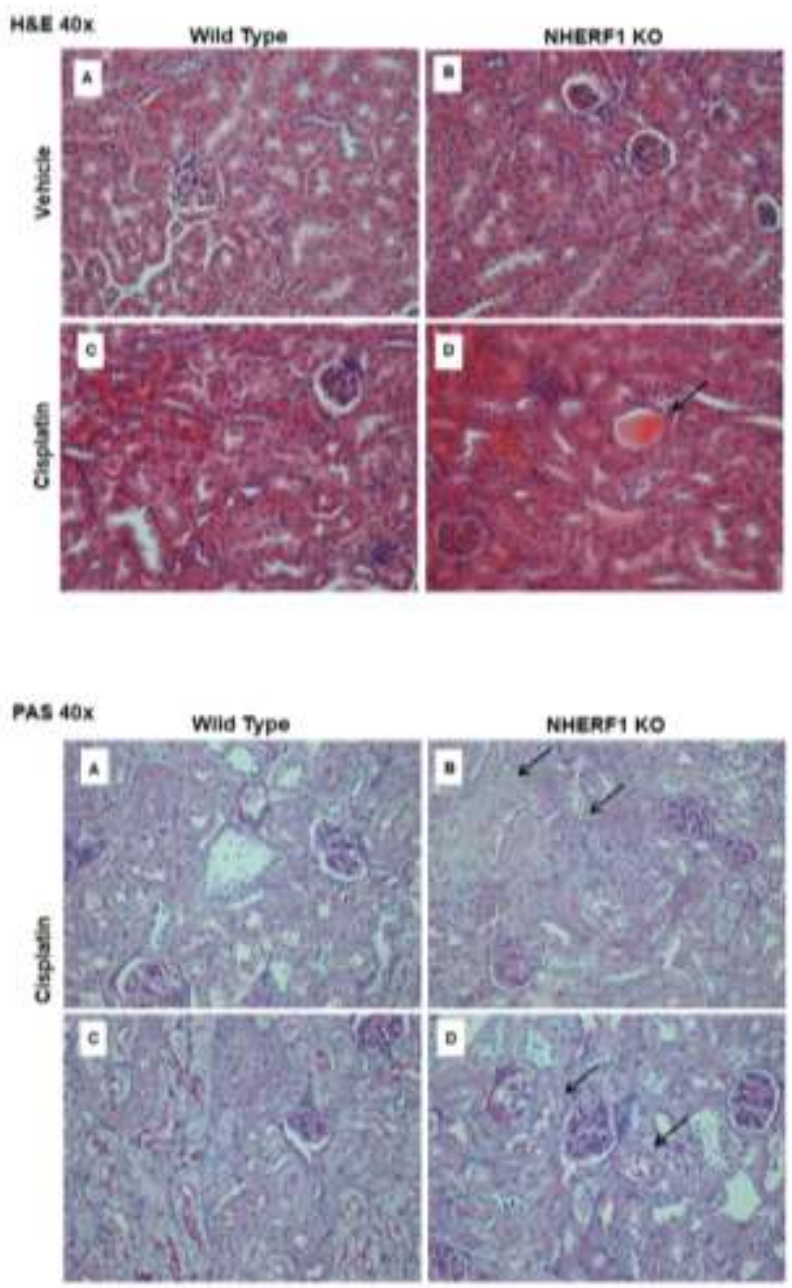

Figure 5.1: Early histologic effect of cisplatin on WT and NHERF1 KO kidneys.

Representative photomicrographs (40x) of H\&E (top panel) and PAS staining (bottom panel) of 24 hour cisplatin treated WT and NHERF1 KO mice. H\&E panel $(A)$ represents a vehicle treated WT kidney, $(B)$ a vehicle treated NHERF1 KO kidney, (C) a cisplatin treated WT kidney, and (D) a cisplatin treated NHERF1 KO kidney. PAS panel (A) and (C) represent cisplatin treated WT kidneys and panel (B) and (D) represent cisplatin treated NHERF1 KO kidneys. Arrows indicate areas of injury (casts and BBM sloughing). 


\section{Figure 5.2}

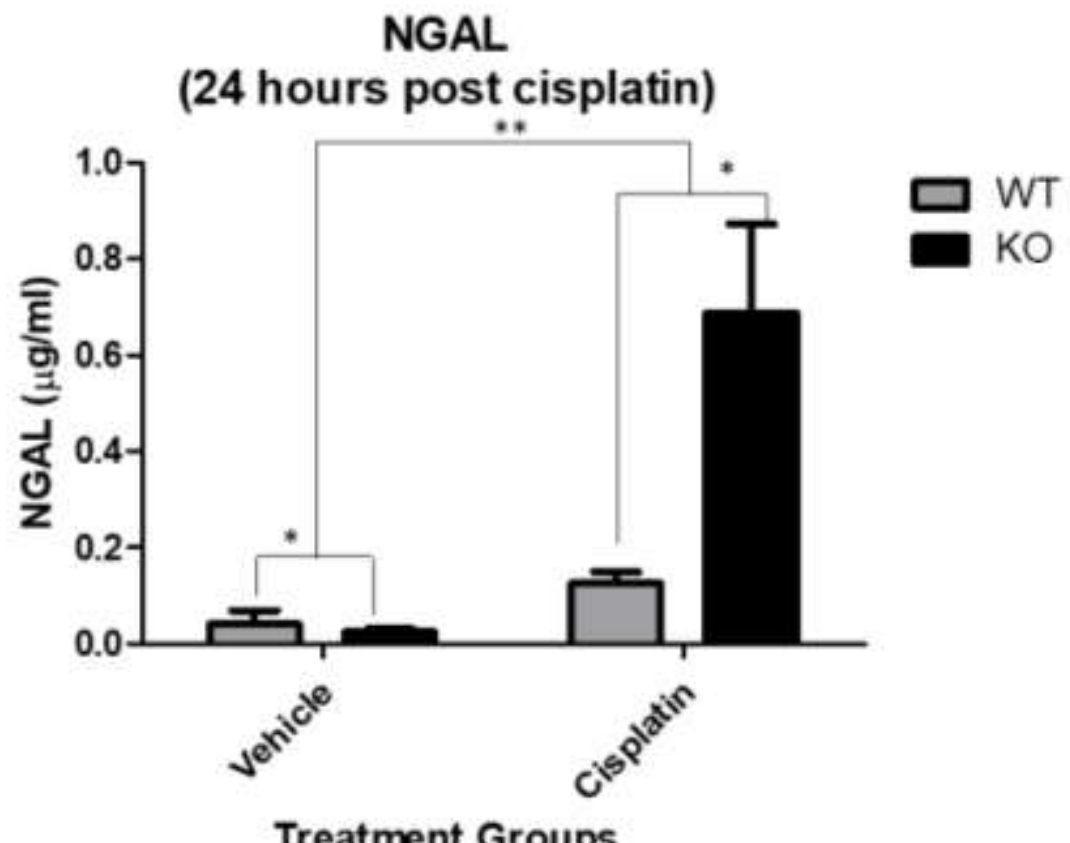

Figure 5.2: Effect of cisplatin on urine NGAL.

NGAL protein measurement of mouse urine in 24 hour cisplatin treated WT and NHERF1 KO mouse kidneys. Data are means \pm SEM (WT vehicle: $n=5$ ), (NHERF1 KO vehicle: $n=5$ ), (WT cisplatin: $n=7$ ), and NHERF1 KO cisplatin: $n=8$ ). ${ }^{* *} P=0.007$ cisplatin treated WT and NHERF1 KO mice compared to vehicle saline controls; ${ }^{*} P=0.03$ cisplatin treated NHERF1 $\mathrm{KO}$ compared to cisplatin treated WT mice; ${ }^{*} P=0.04$ vehicle treated NHERF1 KO mice compared to vehicle treated WT mice. 


\section{NHERF1 loss does not affect lipid peroxidation by TBARS}

Cisplatin nephrotoxicity has been shown in rats to induce lipid peroxidation, oxidative degeneration on lipids in cellular membranes (120). A TBARS assay was utilized to test if NHERF1 loss alone led to lipid peroxidation or if cisplatin treated NHERF1 KO mice had an increased level of lipid peroxidation. The TBARS assay measures levels of malondialdehyde (MDA) and other lipid peroxidation products. WT and NHERF1 KO 72 hour cisplatin treated kidney cortex homogenates were used for this assay. Under baseline conditions lipid peroxidation levels were similar for both genotypes (WT vehicle: $0.95 \mathrm{nmole} / \mathrm{mg}$ \pm 0.09 ) and (NHERF1 KO vehicle: $0.96 \mathrm{nmole} / \mathrm{mg} \pm 0.09$ ) (Figure 5.3). No significant increase of lipid peroxidation was found with cisplatin treatment in either WT $(0.86 \mathrm{nmole} / \mathrm{mg} \pm 0.05)$ or NHERF1 KO $(0.83 \mathrm{nmole} / \mathrm{mg} \pm 0.07)$ mice (Figure 5.3). Furthermore, no differences were found between cisplatin treated WT and NHERF1 KO mice (Figure 5.3).

\section{NHERF1 loss increases 4-hydroxynonenal in cortex and juxtamedullary region following cisplatin treatment}

Besides MDA, 4-hydroxynonenal (4-HNE) is another product of lipid peroxidation. To give a broader view of lipid peroxidation in vehicle and cisplatin treated mice IHC of 4-HNE was performed. IHC for 4-HNE showed minimal staining with no marked differences between genotypes for vehicle treated kidneys (Figure 5.4). Cisplatin treatment resulted in an increase in 4-HNE 
Figure 5.3

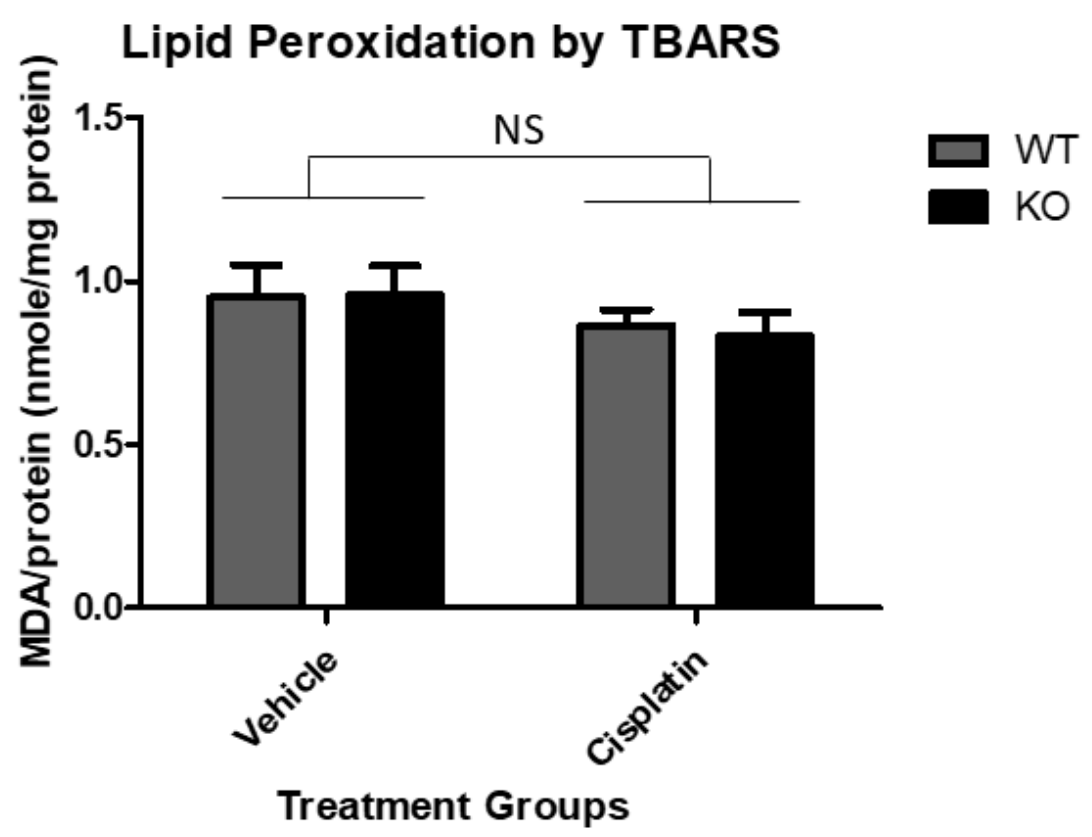

Figure 5.3: Cisplatin's effect on lipid peroxidation by TBARS in WT and NHERF1 KO mice.

TBARS measurement of lipid peroxidation of kidney cortex 72 hour cisplatin treated WT and NHERF1 KO mice. Data are means \pm SEM (WT vehicle: $n=5$ ), (NHERF1 KO vehicle: $n=5$ ), (WT cisplatin: $n=8$ ), and NHERF1 KO cisplatin: $n=9$ ). No significant differences were found between treatment groups. 


\section{Figure 5.4}

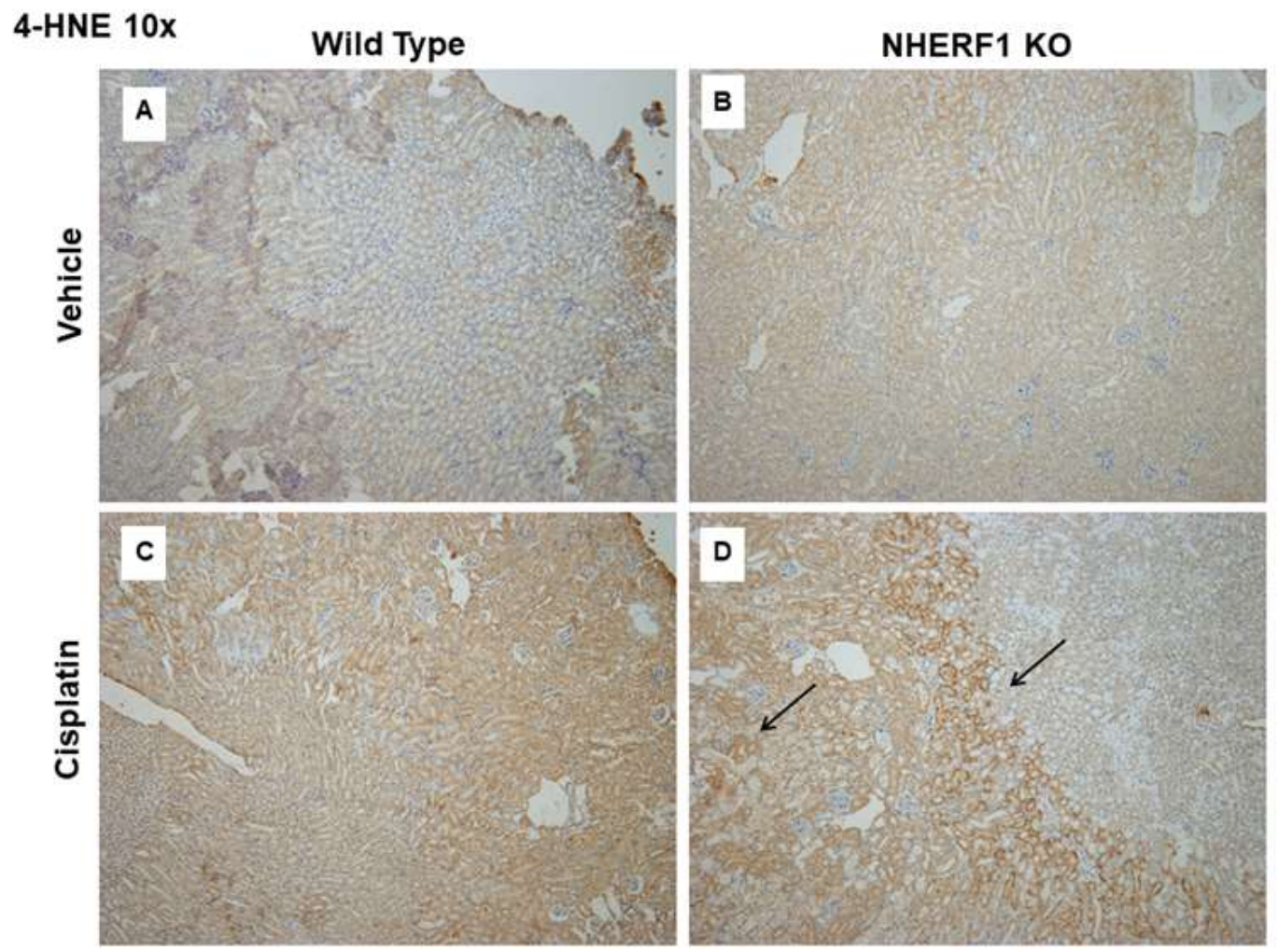

Figure 5.4: The effect of NHERF1 loss and cisplatin treatment on 4-HNE staining in the kidney.

Representative photomicrographs (10x) of IHC staining of 4-HNE in 72 hour cisplatin treated WT and NHERF1 KO mice. Panel (A) represents a vehicle treated WT kidney, (B) a vehicle treated NHERF1 KO kidney, (C) a cisplatin treated WT kidney, and (D) a cisplatin treated NHERF1 KO kidney. Arrows indicate areas of marked staining in the cortex and juxtamedullary (JM) region of the kidney. 
staining within the cortex and juxtamedullary (JM) region of both genotypes, however, staining was more marked in the cisplatin treated NHERF1 KO kidneys, especially in the JM regions (Figure 5.4).

\section{NHERF1 KO kidneys do not have altered GSH metabolism}

Cisplatin nephrotoxicity is proposed to deplete GSH and to induce oxidative stress resulting in cellular injury and death. Due to this finding, HPLC of the reduced and oxidized forms of small molecular weight thiols, GSH, glutathione disulfide (GSSG), cysteine (Cys) and cystine (CySS), and the mixed disulfide between the two, cysteine-glutathione disulfide (CySSG), were performed on plasma and kidney cortex 72 hours after cisplatin treatment. In vehicle treated mice, the only significant difference observed in plasma was a CySS decrease in NHERF1 KO $(24.1 \mu \mathrm{M} \pm 3.7)$ relative to $\mathrm{WT}(37.6 \mu \mathrm{M} \pm 2.7)(P=0.0042)$ (Figure 5.5 A). Additionally, after cisplatin treatment NHERF1 KO mice $(18.9 \mu \mathrm{M} \pm 3.2)$ had a decrease in plasma CySS compared to WT $(29 \mu \mathrm{M} \pm 4)(P=0.0042)$ (Figure 5.5 A). In response to cisplatin, plasma CySS, Cys [(WT vehicle: $29 \mu \mathrm{M} \pm$ 3), (NHERF1 KO vehicle: $31 \mu \mathrm{M} \pm 2$ ), (WT cisplatin: $23 \mu \mathrm{M} \pm 3$ ), (NHERF1 KO cisplatin: $20 \mu \mathrm{M} \pm 2)](P=0.013)$, CySSG [(WT vehicle: $22 \mu \mathrm{M} \pm 3)$, (NHERF1 KO vehicle: $21 \mu \mathrm{M} \pm 6$ ), (WT cisplatin: $16 \mu \mathrm{M} \pm 1$ ), (NHERF1 KO cisplatin: $10 \mu \mathrm{M}$ $\pm 2)](P=0.003), \mathrm{GSH}$ [(WT vehicle: $30 \mu \mathrm{M} \pm 3)$, (NHERF1 KO vehicle: $35 \mu \mathrm{M} \pm$ 2), (WT cisplatin: $23 \mu \mathrm{M} \pm 2$ ), (NHERF1 KO cisplatin: $24 \mu \mathrm{M} \pm 4)](P=0.02)$, and GSSG [(WT vehicle: $5 \mu \mathrm{M} \pm 1$ ), (NHERF1 KO vehicle: $7 \mu \mathrm{M} \pm 2$ ), (WT cisplatin: 3 $\mu \mathrm{M} \pm 0.3)$, (NHERF1 KO cisplatin: $3 \mu \mathrm{M} \pm 0.6)](P=0.002)$ decreased when 
Figure 5.5

A.

Plasma Cysteine

(72 hour post cisplatin)

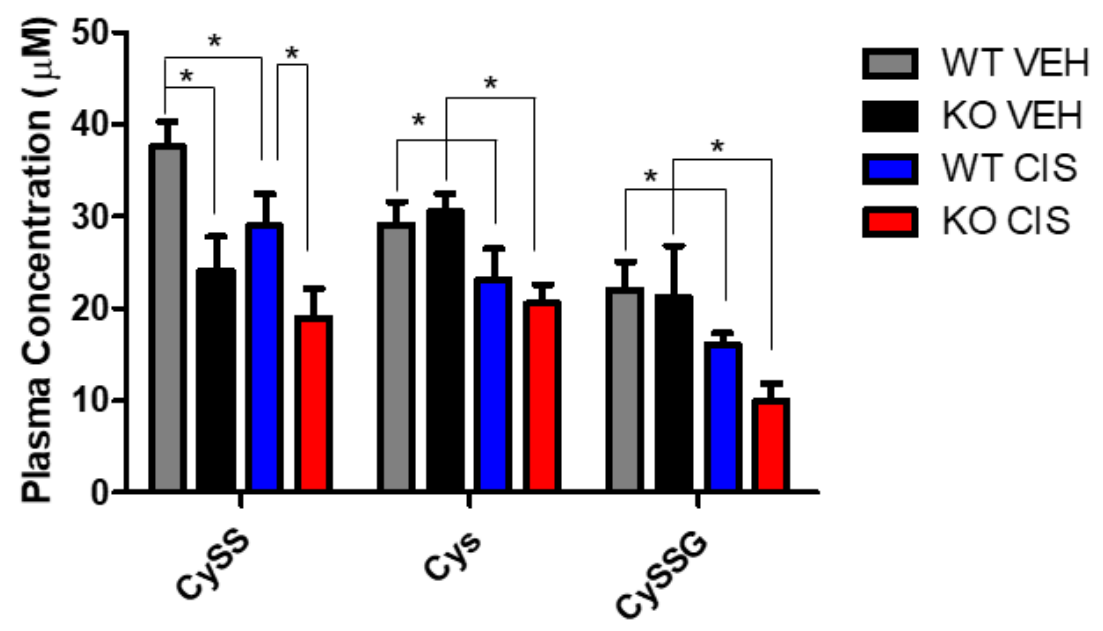

B.

Plasma Glutathione (72 hour post cisplatin)

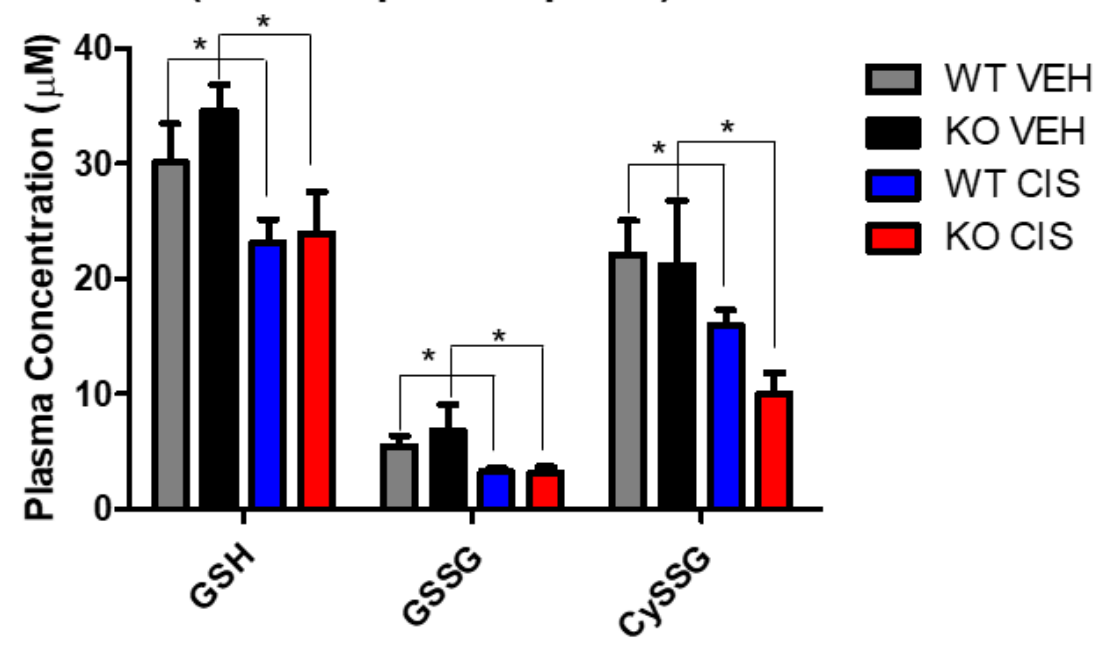


c.

Kidney Cysteine

(72 hour post Cisplatin)

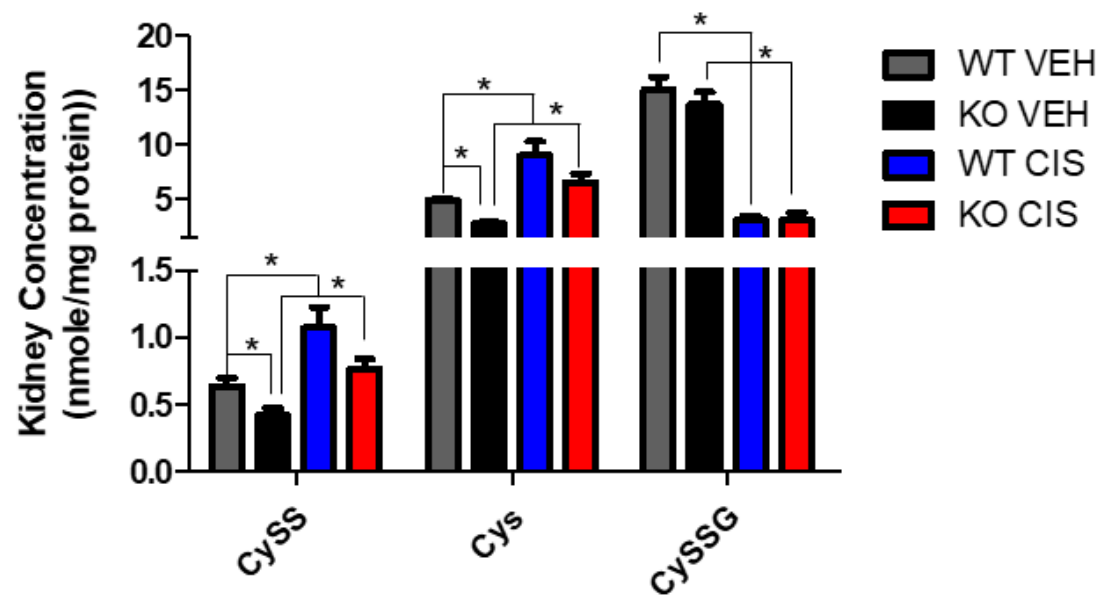

D.

Kidney Glutathione

(72 hour post Cisplatin)

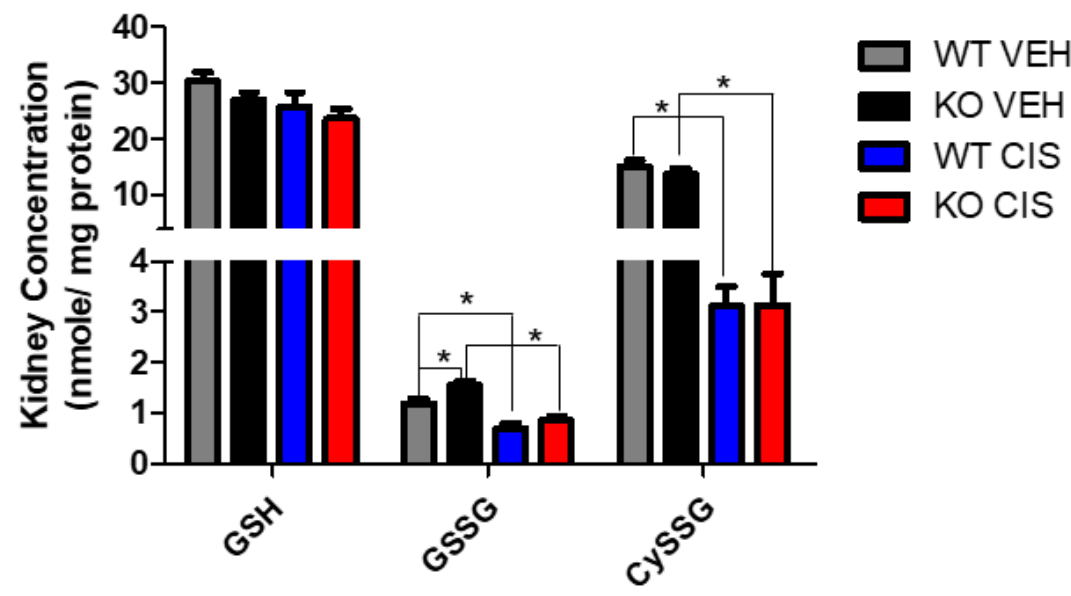


E.

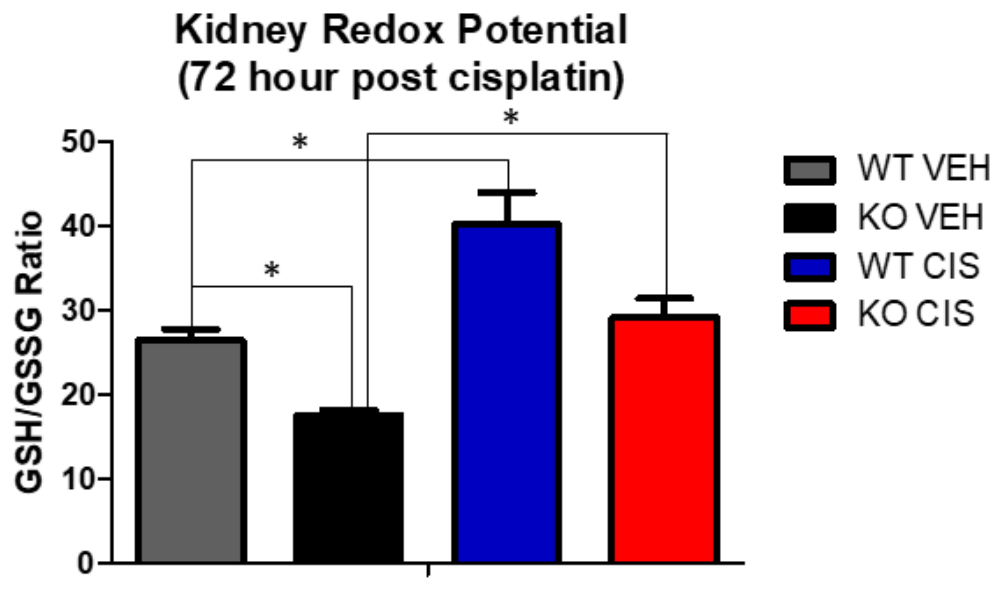

Figure 5.5: Cisplatin and NHERF1 loss effect on small molecular weight thiols. Plasma and Kidney GSG, GSSG, CySSG, Cys, and CySS were measured in 72 hour vehicle and cisplatin treated WT and NHERF1 KO mice. Data are means \pm SEM. Plasma: (WT vehicle: $n=5)$, (NHERF1 KO vehicle: $n=5)$, (WT cisplatin: $n=7$ ), and (NHERF1 KO cisplatin: $n=9$ ) and Kidney: (WT vehicle: $n=5),(N H E R F 1$ KO vehicle: $n=5$ ), (WT cisplatin: $n=8$ ), and (NHERF1 KO cisplatin: $n=9$ ). Plasma: ${ }^{*} P<0.05$ cisplatin treated WT and NHERF1 KO mice compared to vehicle saline controls for CySS, Cys, CySSG, GSH, GSSG, and CySSG; * $P<0.05$ vehicle treated NHERF1 KO compared to vehicle treated WT mice for CySS. Kidney: ${ }^{*} P$ $<0.05$ vehicle treated NHERF1 KO mice compared to vehicle treated WT mice for CySS, Cys, and GSSG; ${ }^{*}<0.05$ cisplatin treated WT and NHERF1 KO mice compared to vehicle saline controls for CySS, Cys, CySSG, and GSSG. 
Table 5.1:

\begin{tabular}{|c|c|c|c|c|c|c|c|c|}
\hline $\begin{array}{c}\text { Oxidized and Reduced } \\
\text { forms }\end{array}$ & \multicolumn{4}{|c|}{ Plasma } & \multicolumn{5}{c|}{ Kidney } \\
\hline & $\begin{array}{c}\text { WT } \\
\text { veh }\end{array}$ & $\begin{array}{c}\text { KO } \\
\text { veh }\end{array}$ & $\begin{array}{c}\text { WT } \\
\text { cis }\end{array}$ & $\begin{array}{c}\text { KO } \\
\text { cis }\end{array}$ & $\begin{array}{c}\text { WT } \\
\text { veh }\end{array}$ & $\begin{array}{c}\text { KO } \\
\text { veh }\end{array}$ & $\begin{array}{c}\text { WT } \\
\text { cis }\end{array}$ & $\begin{array}{c}\text { KO } \\
\text { cis }\end{array}$ \\
\hline CyS & $\uparrow$ & $\uparrow$ & $\downarrow$ & $\downarrow$ & $\uparrow$ & $\downarrow$ & $\uparrow$ & $\uparrow$ \\
\hline CySS & $\uparrow$ & $\downarrow$ & $\uparrow$ & $\downarrow$ & $\uparrow$ & $\downarrow$ & $\uparrow$ & $\uparrow$ \\
\hline CySSG & $\uparrow$ & $\uparrow$ & $\downarrow$ & $\downarrow$ & $\uparrow$ & $\uparrow$ & $\downarrow$ & $\downarrow$ \\
\hline GSH & $\uparrow$ & $\uparrow$ & $\downarrow$ & $\downarrow$ & NS & NS & NS & NS \\
\hline GSSG & $\uparrow$ & $\uparrow$ & $\downarrow$ & $\downarrow$ & $\downarrow$ & $\uparrow$ & $\downarrow$ & $\downarrow$ \\
\hline GSH/GSSG & N/A & N/A & N/A & N/A & $\uparrow$ & $\downarrow$ & $\uparrow$ & $\uparrow$ \\
\hline
\end{tabular}

Table 5.1: Summary of the effect of NHERF1 and cisplatin on small molecular weight thiols.

Plasma and Kidney GSG, GSSG, CySSG, Cys, and CySS were measured in 72 hour vehicle and cisplatin treated WT and NHERF1 KO mice. Table 5.1 illustrates the summarized effects of NHERF1 and cisplatin treatment on CyS, CySS, CySSG, GSH, GSSG, and GSH/GSSG in both the plasma and the kidney. N/A stands for not applicable and NS for not significant. Arrows indicate increased or decreased levels of each small molecular weight thiol. 
compared to vehicle treated mice (Figure 5.5A \& B). In the kidney vehicle treated NHERF1 KO mice had a decrease in Cys ( $3 \mathrm{nmole} / \mathrm{mg}$ protein \pm 2$)(P=0.03)$ and an increase in GSSG (2 nmole/mg protein \pm 0.1$)(P=0.022)$ relative to vehicle treated WT mice [(CySSG: $15 \mathrm{nmole} / \mathrm{mg}$ protein \pm 1$)$, (Cys: $5 \mathrm{nmole} / \mathrm{mg}$ protein \pm 0.2 ), (GSSG: 1 nmole/mg protein \pm 0.1 ) (Figure $5.5 \mathrm{C} \& \mathrm{D})$. Cisplatin treatment led to an increase in CySS [(WT cisplatin: $1 \mathrm{nmole} / \mathrm{mg}$ protein \pm 0.1 ), (NHERF1 KO cisplatin: $0.8 \mathrm{nmole} / \mathrm{mg}$ protein \pm 0.08$)](P=0.002)$ and Cys [(WT cisplatin: 9 nmole/mg protein \pm 1 ), (NHERF1 KO cisplatin : 7 nmole/mg protein \pm $0.8)](P=0.0005)$ and a decrease in CySSG [(WT cisplatin: 3 nmole/mg protein \pm 0.4), (NHERF1 KO cisplatin: 3 nmole/mg protein \pm 0.7$)](P<0.0001)$ and GSSG [(WT cisplatin: $0.7 \mathrm{nmole} / \mathrm{mg}$ protein \pm 0.1$),(\mathrm{NHERF} 1 \mathrm{KO}$ cisplatin: $0.8 \mathrm{nmole} / \mathrm{mg}$ protein \pm 0.08$)](P<0.0001)$ in both genotypes (Figure $5.5 \mathrm{C} \& \mathrm{D})$. On the other hand, cisplatin did not affect the kidney levels of GSH [(WT vehicle: 30 nmole/mg protein \pm 2 ), (NHERF1 KO vehicle: $27 \mathrm{nmole} / \mathrm{mg}$ protein \pm 1 ), (WT cisplatin: 26 nmole/mg protein \pm 3 ), (NHERF1 KO cisplatin: 24 nmole/mg protein \pm 2$)$ ] in either genotype (Figure 5.5 D). Kidney redox potential (GSH/GSSG) was also measured and found to be increased in both genotypes with cisplatin treatment [(WT vehicle: $26.4 \pm 1.4)$, (NHERF1 KO vehicle: $18 \pm 0.7)$, (WT cisplatin: $40.2 \pm$ 4), and (NHERF1 KO cisplatin: $29.2 \pm 2)](P=0.0002)$ (Figure $5.5 \mathrm{E})$. Furthermore, NHERF1 KO kidneys were found to have a decrease in GSH/GSSG redox potential under baseline $(P=0.003)$ (Figure $5.5 \mathrm{E})$.

\section{NHERF1 loss affects GGT activity response to cisplatin insult}


Although controversial, GGT activity is thought to be part of cisplatin's biotransformation into a nephrotoxin. Urine GGT activity was found to be highest after cisplatin treatment at 24 hours or earlier in humans (154). To understand the effect NHERF1 loss has on GGT activity in response to cisplatin over time 4 $\mathrm{hr}$ and $24 \mathrm{hr}$ cisplatin treated WT and NHERF1 KO kidneys were evaluated. GGT activity was found to be non-significantly altered with NHERF1 loss alone at any timepoint [4hr (WT vehicle: $5800 \mathrm{nmole} / \mathrm{min} / \mathrm{mL} \pm$ 1300), (NHERF1 KO vehicle: $5700 \mathrm{nmole} / \mathrm{min} / \mathrm{mL} \pm 1600$ )] and [24 hr (WT vehicle: $8100 \mathrm{nmole} / \mathrm{min} / \mathrm{mL} \pm$ 2300), (NHERF1 KO vehicle: $7200 \mathrm{nmole} / \mathrm{min} / \mathrm{mL} \pm 1500)$ ], and remained similar 4 hours after cisplatin treatment [4 hr (WT cisplatin: $7000 \mathrm{nmole} / \mathrm{min} / \mathrm{mL} \pm 1600$ ), (NHERF1 KO cisplatin: $5300 \mathrm{nmole} / \mathrm{min} / \mathrm{mL} \pm$ 1600)] (Figure 5.6). However, 24 hours after cisplatin treatment there was a significant genotype difference between WT and NHERF1 KO kidneys in response to cisplatin [24 hr (WT cisplatin: $9900 \mathrm{nmole} / \mathrm{min} / \mathrm{mL} \pm 2400$ ), (NHERF1 KO cisplatin: 7300 nmole $/ \mathrm{min} / \mathrm{mL} \pm 1400)](P=0.04)$ (Figure 5.6).

\section{NHERF1 loss does not affect GGT protein expression}

To evaluate if NHERF1 loss affected the amount of GGT protein a western blot was performed on the 4 and 24 hour cisplatin treated WT and NHERF1 KO kidney homogenates (Figure 5.7). GGT protein expression was found to be unchanged with NHERF1 loss under baseline conditions for either time point [4 hr (WT vehicle: 0.84 GGT/GAPDH \pm 0.05), (NHERF1 KO vehicle: 0.62 GGT/GAPDH \pm 0.08$)]$ and 


\section{Figure 5.6}

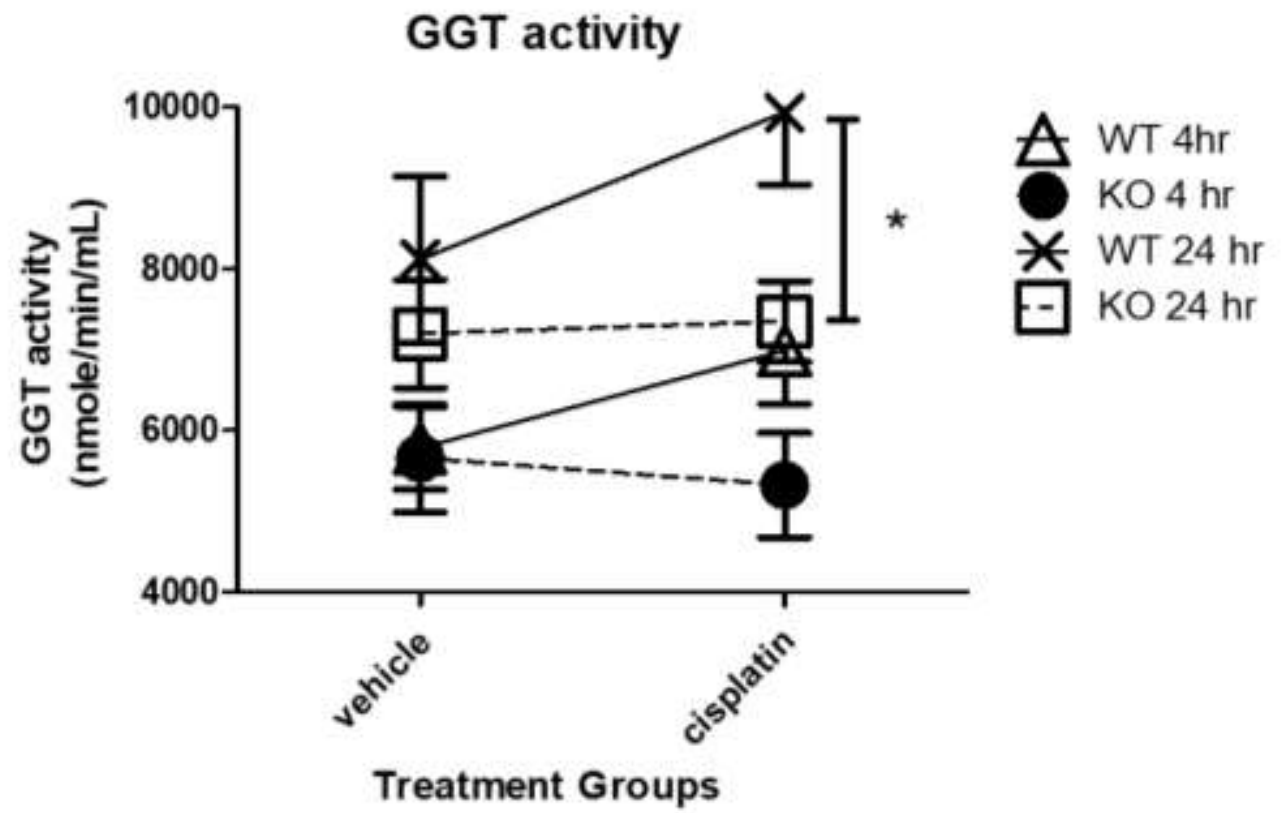

Figure 5.6: Effect of NHERF1 on GGT activity following cisplatin treatment.

GGT activity was measured at 4 hours and 24 hours after cisplatin treatment in WT and NHERF1 KO kidney cortex homogenates as described in Chapter II. Data are means \pm SEM. 4 hours: (WT vehicle: $n=6)$, (NHERF1 KO vehicle: $n=6$ ), (WT cisplatin: $n=6$ ), and (NHERF1 KO cisplatin: $n=6$ ) and 24 hours: (WT vehicle: $n=5)$, (NHERF1 KO vehicle: $n=5)$, (WT cisplatin: $n=7)$, and (NHERF1 KO cisplatin: $\mathrm{n}=8$ ). ${ }^{*} P<0.04$ cisplatin treated NHERF1 KO compared to cisplatin treated WT. 


\section{Figure 5.7}
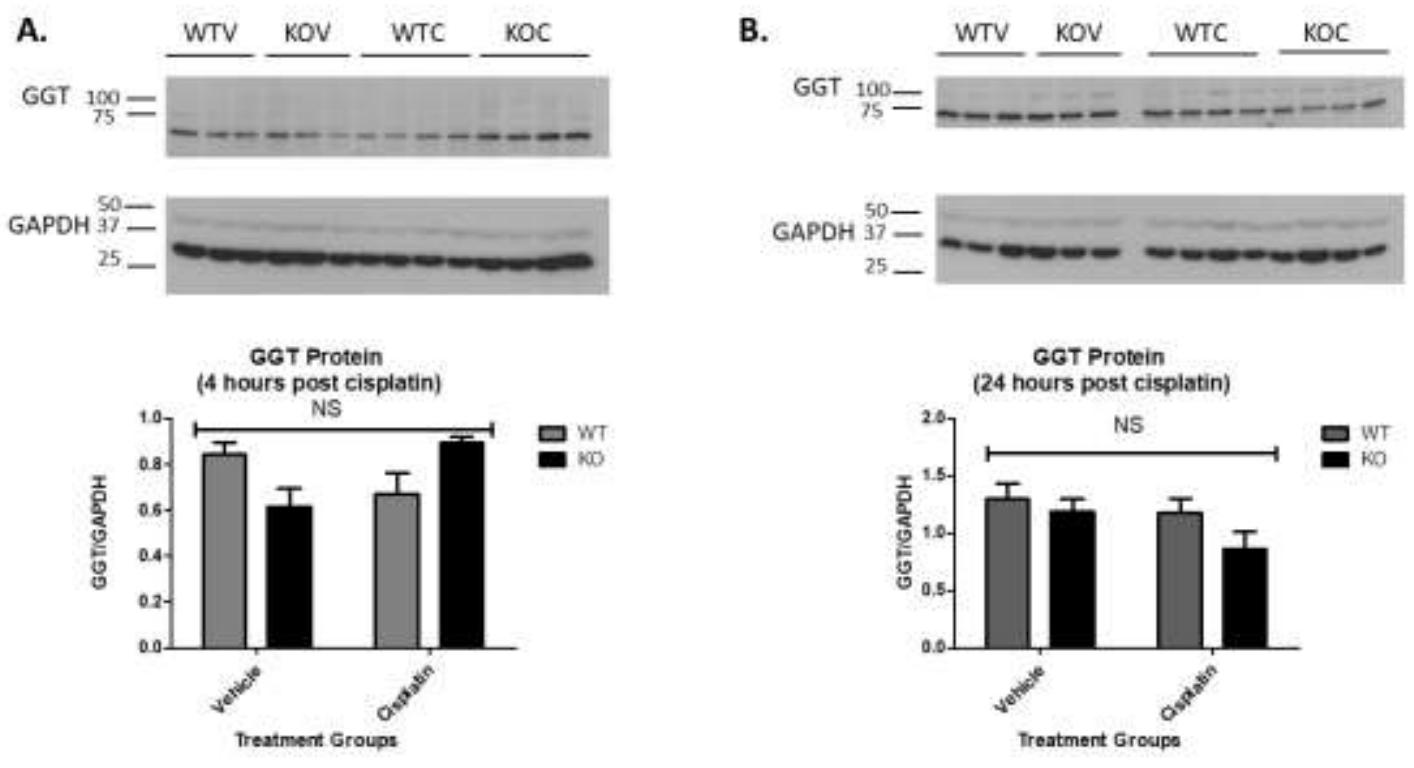

Figure 5.7: Effect of NHERF1 loss on GGT protein.

Representative blots of 4 hour (A) and 24 hour (B) GGT and GAPDH are shown. Kidney cortex homogenates were separated by $10 \%$ SDS-PAGE and transferred to nitrocellulose membranes. Quantitation was performed as described in Chapter II (B) Data are means \pm SEM (WT vehicle: $n=3)$, (NHERF1 vehicle: $n=3$ ), (WT cisplatin: $n=4$ ), and (NHERF1 KO cisplatin: $n=4)$. A representative blot from 2 independent experiments for each time point is shown. 
[24 hr (WT vehicle: 1.3 GGT/GAPDH \pm 0.14), (NHERF1 KO vehicle: 1.2 GGT/GAPDH \pm 0.1)] (Figure 5.7). Following cisplatin the amount of GGT protein was also found to be unchanged at 4 and 24 hours post treatment in both genotypes [4 hr (WT cisplatin: $0.7 \mathrm{GGT} / \mathrm{GAPDH} \pm$ 0.09), (NHERF1 KO cisplatin:

0.9 GGT/GAPDH \pm 0.03 )] and [24 hr (WT cisplatin: 1.2 GGT/GAPDH \pm 0.13 ), (NHERF1 KO vehicle: 0.9 GGT/GAPDH \pm 0.2)] (Figure 5.7).

\section{GGT localization is altered in NHERF1 KO kidneys 24 hours after cisplatin treatment}

To evaluate localization of GGT in WT and NHERF1 KO kidneys, kidney slices were stained for GGT by IHC. IHC revealed similar GGT staining in the proximal tubules of both vehicle treated genotypes (Figure 5.8, panel A \& B). In cisplatin treated WT kidneys there was no apparent damage and GGT localization remained unaltered (Figure 5.8, panel C). However, in the cisplatin treated NHERF1 KO kidneys areas of BBM sloughing in the proximal tubule showed decreased staining for GGT (Figure 5.8, panel D).

\section{NHERF1 loss does not affect CCBL protein}

CCBL is a pyridoxal phosphate-dependent enzyme that metabolizes Scysteine conjugates through transamination or $\beta$-elimination reactions, and is also proposed to be involved in cisplatin's biotransformation into a nephrotoxin. To determine if there were any changes in CCBL protein in WT and NHERF1 KO mice 4 hour and 24 hour cisplatin treated kidney cortex 


\section{Figure 5.8}

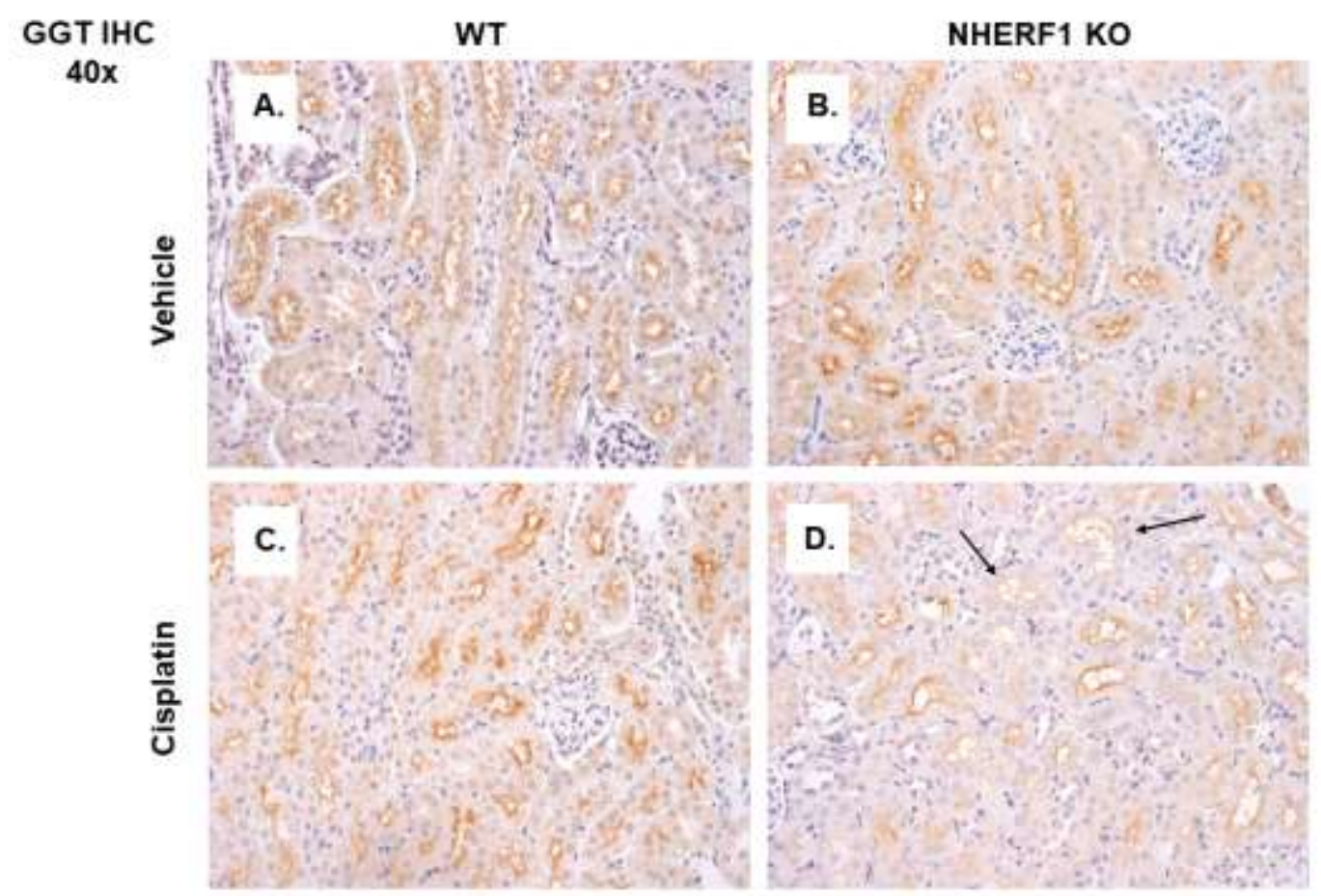

Figure 5.8: GGT localization in vehicle and cisplatin treated kidneys.

Representative photomicrographs (40x) of IHC staining of GGT in 24 hour cisplatin treated WT and NHERF1 KO mice. Panel (A) represents a vehicle treated WT kidney, (B) a vehicle treated NHERF1 KO kidney, (C) a cisplatin treated WT kidney, and (D) a cisplatin treated NHERF1 KO kidney. Arrows indicate areas of BBM sloughing and GGT staining loss. 
homogenates were analyzed via western blot. CCBL protein was found to be unchanged with NHERF1 loss under baseline conditions for either time point [4 hr (WT vehicle: 1.4 CCBL/GAPDH \pm 0.3$)$, (NHERF1 KO vehicle: 1.1 $\mathrm{CCBL} / \mathrm{GAPDH} \pm .01)$ ] and [24 $\mathrm{hr}$ (WT vehicle: $2.5 \mathrm{CCBL} / \mathrm{GAPDH} \pm 0.7$ ), (NHERF1 KO vehicle: 1.2 CCBL/GAPDH \pm 0.1 )] (Figure 5.9). Following cisplatin the amount of CCBL protein was also found to be unchanged at 4 and 24 hours post treatment in both genotypes [ $4 \mathrm{hr}$ (WT cisplatin: 1.7 CCBL/GAPDH \pm 0.1 ), (NHERF1 KO cisplatin: 1.3 CCBL/GAPDH \pm 0.2)] and [24 hr (WT cisplatin: 2.2 CCBL/GAPDH \pm 0.4 ), (NHERF1 KO cisplatin: $2.4 \mathrm{CCBL} / \mathrm{GAPDH} \pm 0.8)$ ] (Figure $5.9)$.

\section{Discussion}

The purpose of this study was to determine if NHERF1 loss affected the renal handling of cisplatin. This possibility was investigated as a potential mechanism of susceptibility of NHERF1 KO mice to cisplatin-induced AKI. This is the first study to investigate the effect NHERF1 loss has on cisplatin uptake and/or metabolism in the kidney as well as the first study to show an altered GGT activity in response to cisplatin in NHERF1 KO mice.

NHERF1 KO mice are indeed more sensitive to cisplatin as confirmed by earlier signs of cisplatin related kidney injury. WT mice did not show histologic signs of injury 24 hours after cisplatin treatment. Additionally, WT mice had a moderate increase in urine NGAL, a sensitive marker of kidney injury, 24 hours after treatment. On the other hand, NHERF1 KO mice showed patchy areas of 


\section{Figure 5.9}
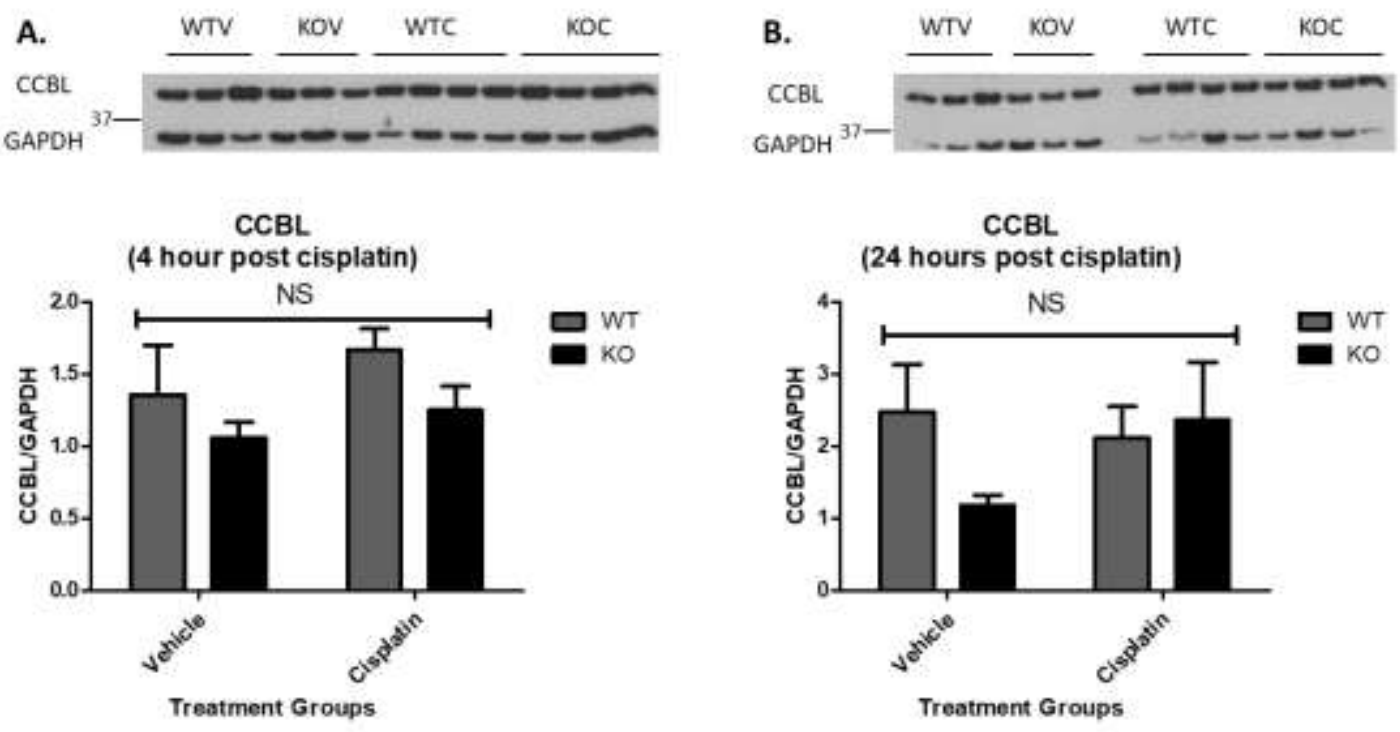

Figure 5.9: Effect of NHERF1 loss on CCBL protein.

Representative blots of 4 hour (A) and 24 hour (B) CCBL and GAPDH are shown. Kidney cortex homogenates were separated by $10 \%$ SDS-PAGE and transferred to nitrocellulose membranes. Quantitation was performed as described in Chapter II (B) Data are means \pm SEM (WT vehicle: $n=3$ ), (NHERF1 vehicle: $n=3$ ), (WT cisplatin: $n=4)$, and (NHERF1 KO cisplatin: $n=4)$. A representative blot from 2 independent experiments for each time point is shown. 
histologic injury 24 hours after cisplatin administration (e.g. BBM sloughing, casts, and atrophy). Moreover, NHERF1 KO mice had a robust increase in urine NGAL 24 hours following cisplatin treatment. These data corroborate the increased sensitivity to cisplatin seen in the previous 72 hour study (35). In addition to evaluating the sensitivity of NHERF1 KO mice to cisplatin, levels of lipid peroxidation were also measured.

Cisplatin has been shown to increase lipid peroxidation due to induced oxidative stress. In this study a TBARS assay was utilized and WT and NHERF1 $\mathrm{KO}$ mice were found to have similar levels of lipid peroxidation via MDA. These data illustrate the perplexing nature of cisplatin nephrotoxicity, where much of the research is contradictory. In this study kidney cortex was used for the sample preparation, however, other studies used whole kidney or did not clarify whether cortex was isolated $(155,156)$. Conceivably these conflicting results may be due to what area of the kidney was used, especially since IHC of 4-HNE revealed that NHERF1 KO mice had an increased positive staining in both cortex and the JM region. On the other hand, many of the lipid peroxidation studies were performed on rats, while studies on mice seem to be dependent on the concentration of cisplatin, dosing regimen, and mouse strain $(116,156,157)$. In addition, rats are more sensitive to cisplatin nephrotoxicity than mice (116). Thus, cisplatin nephrotoxic cell death and the relationship to induced lipid peroxidation remains obscure. However, both the TBARS assay and the IHC data reinforce that NHERF1 KO mice do not have an underlying increase in lipid peroxidation. 
Therefore, this is not a mechanism of susceptibility of NHERF1 KO mice to cisplatin-induced AKI.

Even though there was no apparent increase in lipid peroxidation other indicators of oxidative stress were measured. These indicators included the oxidized and reduced forms of small molecular weight thiols that act as antioxidants. Plasma from 72 hour cisplatin treated mice showed a consistent decrease in GSH, GSSG, CySSG, Cys, and CySS. These data are consistent with other reports $(25,158-160)$. When compared to WT alone, NHERF1 loss led to a decrease in cystine (CySS), which is the oxidized form of cysteine. CySS is readily taken up into cells to be reduced to Cys. Cys is a necessary substrate for the cellular antioxidant GSH (161). Although plasma CySS is decreased in the vehicle treated NHERF1 KO mice GSH in the kidney remains similar between vehicle treated WT and NHERF1 KO mice. Like in other studies, the kidney CySSG and GSSG are decreased in both genotypes with cisplatin treatment, but, unexpectedly GSH was not decreased in either genotype (159). The oxidized form of glutathione (GSSG), was increased in the kidney of vehicle treated NHERF1 KO mice. Collectively, NHERF1 KO mice had a decrease in CySS in the plasma and an increase in GSSG in the kidney. Moreover, NHERF1 KO mice had a decrease in kidney redox potential (GSH/GSSG) when compared to WT. Perhaps these data indicate a low level of oxidative stress or maybe a suppressed ability to convert CySS to Cys and GSSG to GSH with NHERF1 loss. However, NHERF1 KO kidneys do not reflect a decrease in GSH levels. Therefore, it appears that NHERF1 loss does not affect GSH metabolism itself. 
Unfortunately, the role of NHERF1 loss and an underlying oxidative stress remains ambiguous as a potential mechanism. In the future measuring glutathione peroxidase, glutathione synthase, superoxide dismutase or catalase in WT and NHERF1 KO mice may be beneficial.

In addition to GSH metabolism and oxidative stress indicators, GGT activity was also measured. Several studies have indicated GGT activity and/or expression is important to the biotransformation of cisplatin to a nephrotoxin (13, $25,26)$. A robust increase in GGT activity was seen in the 24 hour cisplatin treated WT mice, while an increase in GGT activity was not seen in the NHERF1 $\mathrm{KO}$ mice, making it appear that they are unable to respond to cisplatin in this manner and suggesting that NHERF1 is important for GGT's response to an insult. The role of GGT activity or expression in protection or susceptibility to cisplatin nephrotoxicity is also controversial $(113,151)$. GGT KO mice are protected from cisplatin nephrotoxicity (112). Alternatively, depending on the cell type an increase in GGT activity may be protective or increase sensitivity to the drug $(151,162)$. These data have led to two alternative theories: [1] GGT is important in the conversion of cisplatin to a nephrotoxin thus inhibition leads to protection or [2] GGT is important for detoxification of xenobiotics and production of GSH, thus increased activity results in increased GSH production that can alleviate some of the induced oxidative stress from cisplatin. The second theory may explain the exacerbated AKI in NHERF1 KO mice. If NHERF1 loss limits the ability of GGT to respond quickly and/or robustly to an insult there may be increased injury. Besides evaluating GGT activity, protein levels and localization 
were investigated. GGT protein levels were not significantly different, however, IHC showed some GGT loss in the proximal tubule due to BBM sloughing. This GGT loss has been recorded in mice treated with cisplatin for 72 hours in addition to a decrease in GGT activity at 72 hours $(116,154,163)$. This observation provides an alternative hypothesis for the difference in GGT activity between WT and NHERF1 KO mice. Because NHERF1 KO mice are beginning to lose GGT through BBM sloughing perhaps this is affecting their level of GGT activity in response to cisplatin. Alternatively, a 12 hour time point may be useful to determine if NHERF1 KO mice have an increase in activity between 4 and 24 hours post cisplatin treatment. This proposal would fall under the hypothesis that an increase in GGT activity leads to worse injury. Thus, NHERF1 KO mice could have an increase in GGT activity earlier and subsequently result in increased damage by 24 hours. In any case more research is needed to understand how cisplatin causes nephrotoxicity and what role GGT plays in that mechanism.

$\mathrm{CCBL}$ is the rate limiting enzyme in the production of the 'reactive thiol' in cisplatin nephrotoxicity $(13,25-27)$. Understanding the effect of NHERF1 loss on CCBL protein level, localization, and activity level are valuable pieces of information. In this work CCBL protein level was investigated and found to be similar regardless of genotype or treatment. Future experiments will also evaluate CCBL activity and localization between vehicle and cisplatin treated WT and NHERF1 KO mice. In addition to CCBL, determining platinum (Pt) levels in cisplatin treated WT and NHERF1 KO mice is imperative. A change in Pt levels between cisplatin treated WT and NHERF1 KO mice would be indicative of either 
altered uptake or extrusion of cisplatin. Blocking of the extrusion transport protein, MATE1, results in increased toxicity from cisplatin (22). Moreover, increased uptake and increased cisplatin concentrations led to worse damage and an increase in necrosis $(28,116)$. Previously, 72 hour cisplatin treated NHERF1 KO mice had an increase in ATN when compared to treated WT mice (35). Thus, these data could suggest higher levels of cisplatin in these kidneys. 72 hour and 24 hour cisplatin treated kidneys have been sent for ICP-MS analysis for Pt levels. This future data could provide more insight into the exacerbated AKI of NHERF1 KO mice.

Ultimately, the mechanism of cisplatin nephrotoxicity remains a mystery, however, it is evident that NHERF1 expression has a vital role in its development. Until the other areas discussed (CCBL localization, activity, and Pt levels) have been investigated the underlying mechanism of susceptibility to cisplatin in NHERF1 KO mice remains unclear. These future experiments are integral in elucidating the mechanism of susceptibility. Continued research and broadening the understanding of cisplatin nephrotoxicity and susceptibility is an encouraging area for the development of new innovative preventative protocols or even therapeutic targets that can be used in the clinic. 
CHAPTER VI

\section{DISCUSSION AND CONCLUSIONS}

\section{A. Restatement of goals and questions}

The overall goal of the work described in this dissertation was to discover new potential mechanisms and hypotheses for susceptibility to cisplatin-induced AKI with NHERF1 loss. The work summarized in Chapter III aimed to test if metabolic changes played a role in the sensitization of the NHERF1 KO mouse kidney to cisplatin insult. Chapter IV aimed to evaluate if NHERF1 KO mice had an underlying mitochondrial dysfunction due to: [1] decreases in mitochondrial oxidative capacity and/or alterations in the structure of NHERF1 KO kidneys [2] decreases in number of mitochondria in NHERF1 KO kidneys. Finally, Chapter V of this dissertation used the proposed mechanism of cisplatin biotransformation to a nephrotoxin to test if NHERF1 loss resulted in altered renal handling of cisplatin. Taken together, these studies provide new insight into the mechanism of susceptibility of NHERF1 loss in cisplatin-induced AKI.

\section{B. Major findings of this dissertation}

1. NHERF1 loss results in altered pentose phosphate enzymes in mice 
The accumulation and bioactivation of cisplatin to a nephrotoxic metabolite has been extensively studied, but requires further clarification for the development of novel therapeutic targets or prevention protocols to be used in the clinic. Recent studies have found that cisplatin alters renal cell metabolism, contributing to injury and the secondary result of CKD development. These studies have provided a new avenue of research for cisplatin-induced AKI development. Cisplatin treatment results in depletion of amino acids in the kidney (86-89), reduces fatty acid oxidation while concomitantly accumulating fatty acids in the kidney $(86,89,91)$, and decreases renal glycolytic enzymes and intermediates of the pentose phosphate pathway $(86,93)$. These observations combined with the increased susceptibility to cisplatin-induced AKI suggested the hypothesis that NHERF1 KO mice have metabolic alterations that predispose them to cisplatin nephrotoxicity. Therefore, the goal of Chapter III was to define metabolic changes in the NHERF1 KO mouse. Overall this study uncovered new areas to investigate in understanding susceptibility to cisplatin-induced AKI with NHERF1 loss.

NHERF1 KO mice did not exhibit changes in gluconeogenic or glycolytic enzyme activity. Indeed, cisplatin treatment resulted in a parallel decrease in FBPase and G6Pase activity in NHERF1 KO and WT mice. Additionally, there were no significant changes with $\mathrm{LDH}$ and MDH activity between non-treated and treated WT and NHERF1 KO mice. These results coincide with previous studies $(86,94)$. NHERF1 KO mice also do not have changes in kidney ATP content when compared to WT. On the other hand, NHERF1 KO mouse kidneys exhibit 
increased activity of ME and G6PD under baseline conditions when compared to WT mouse kidneys. Cisplatin treatment resulted in a decrease in G6PD and ME activity in the NHERF1 KO kidneys that was equivalent to the level of cisplatin treated WT kidneys. These findings suggest that NHERF1 KO mice have developed compensatory mechanisms involving the pentose phosphate pathway and in NADPH production in order to maintain the cellular redox state. A similar observation has been made in studies of liver cirrhosis in rats subject to oxidative stress, where an increase in ME and G6PD gene expression and activity (164) are also seen, presumably providing protection against the stress through an increased production of NADPH (164).

These observations bring into question whether NHERF1 KO mice are under oxidative stress that is masked due to increased NADPH. Therefore, when these mice are given cisplatin, a known oxidative stress inducer, the mouse kidneys undergo more severe damage due to the inability to combat the additional underlying oxidative stress following cisplatin treatment. This work has revealed new areas to investigate and laid the foundation for future studies, such as investigating NADPH levels and gene and protein expression of ME and G6PD between WT and NHERF1 KO mice.

\section{Mechanism of susceptibility of NHERF1 KO mice to cisplatin-induced} AKI is not mitochondrial dysfunction

Chapter IV of this dissertation aimed to characterize any differences in mitochondrial function and/or structure between WT and NHERF1 KO mice. This 
aim builds upon the work described in Chapter III of this dissertation, which demonstrated that NHERF1 KO mice do not have gluconeogenic or glycolytic enzyme activity alterations, but do have increased pentose phosphate enzyme activity. In addition to affecting metabolic pathways cisplatin nephrotoxicity has been established in inducing apoptotic and necrotic cell death. The mechanisms involved in cisplatin-induced nephrotoxic cell death remain unclear. However, there is increasing evidence that ROS and mitochondrial function have an important role in cisplatin's mechanism of injury. As discussed in Chapter III, NHERF1 KO mice may have increased levels of NADPH to maintain cellular redox levels, therefore the results from Chapter III and previous studies from our laboratory (poor survival of NHERF1 deficient OK cells and decreased expression of mitochondrial BBM proteins in NHERF1 KO mice) suggested the hypothesis that NHERF1 loss may be associated with mitochondrial dysfunction and susceptibility to kidney injury.

In Chapter IV there were two main goals in characterizing differences in mitochondria between WT and NHERF1 KO mice: [1] to determine if NHERF1 KO mice have altered mitochondrial function and/or structure and [2] to determine NHERF1 KO mice have an underlying mitochondrial dysfunction and if that predisposes these animals to cisplatin nephrotoxicity. EM analysis revealed that NHERF1 KO mice have similar proximal tubule mitochondrial morphologies, size, and distribution and number when compared to WT. Furthermore, there were no overt signs of oxidative stress in NHERF1 KO mitochondria. In fact, only early ischemic changes most likely due to harvesting were noted. In addition, 
isolated mitochondria from NHERF1 KO kidneys were found to have similar oxidative capacities as demonstrated by nonsignificant changes in OCR and similar RCR (WT: 1.63 and KO: 1.61) (Figure 4.3C) to those of WT mice. The $\mathrm{RCR}$ data is compelling since this is the best general measure of mitochondrial function in isolated mitochondria. RCR gives an idea into the mitochondria's ability to respond robustly to the addition of substrate and ADP through the production of ATP. In addition, the RCR value is unique to each tissue and substrate used (146). Therefore, NHERF1 KO mitochondria when isolated from their environment function normally. This study answers an important aspect in evaluating NHERF1 KO mitochondria which is that they can function normally when given optimal components. However, this poses the question if NHERF1 $\mathrm{KO}$ mitochondria function normally in their natural environment. NHERF1 KO mice undergo phosphate wasting (148) potentially creating an intracellular environment where mitochondria cannot function properly. This work uncovers a novel potential mechanism of susceptibility to cisplatin-induced AKI that can be investigated in future studies. Seahorse XF24 analysis on kidney tissue would be beneficial in establishing if NHERF1 KO mice have mitochondrial dysfunction due to phosphate wasting that sensitizes the kidney to cisplatin toxicity.

\section{NHERF1 loss affects GGT activity in response to cisplatin}

The studies in Chapter III and Chapter IV focus on potential underlying changes that could increase susceptibility to cisplatin nephrotoxicity. Therefore, Chapter V focused on the renal handling of cisplatin between WT and NHERF1 
KO mice. This study was based on the hypothesis that NHERF1 loss results in altered renal uptake and/or metabolism of cisplatin resulting in increased toxicity. Understanding the renal handling of cisplatin is an important area of research that has been substantially investigated. Unfortunately, many aspects of cisplatin's metabolism to a nephrotoxin remain ambiguous with conflicting results. For example, the role of GGT activity in cisplatin nephrotoxicity is controversial, where enhanced GGT activity may either increase or decrease sensitivity to cisplatin-induced AKI (151). Furthermore, in the proposed hypothesis of cisplatin's biotransformation to a nephrotoxin, cisplatin is thought to exit the proximal tubule via MATE1 where it is further processed in the tubular lumen and taken up in the proximal tubule by an unknown transport protein. Thus, the conflicting data along with potentially other unknown players add a degree of complexity in understanding cisplatin-induced AKI.

Before investigating changes in renal handling of cisplatin, Chapter $V$ first set to establish that NHERF1 KO mice are indeed more sensitive to the initial cisplatin insult. Therefore, WT and NHERF1 KO mice were treated with cisplatin for 24 hours instead of 72 hours. These kidneys were processed for histology and markers of kidney injury. Ultimately, NHERF1 KO mouse kidneys were confirmed to be more sensitive to the initial insult, characterized by early histologic changes (casts and BBM sloughing) along with significantly increased urine NGAL protein when compared to cisplatin treated WT.

Next, levels of lipid peroxidation were assessed by the means of TBARS analysis and IHC of 4-HNE between vehicle and cisplatin treated WT and 
NHERF1 KO mice. Some studies have shown that cisplatin induces lipid peroxidation, and are thought to be involved in ROS induced cell death (165). NHERF1 KO mouse kidneys were found to not have increased levels of lipid peroxidation under baseline conditions, however, with cisplatin treatment IHC analysis revealed increased positive staining for 4-HNE in the cortex and the JM regions of the kidney. Even though there was no apparent increase in lipid peroxidation other indicators of oxidative stress were measured. These indicators included the oxidized and reduced forms of small molecular weight thiols that act as antioxidants. Plasma from 72 hour cisplatin treated mice showed a decrease in all reduced and oxidized forms of glutathione and cysteine which is consistent with other reports $(25,158-160)$. The only difference noted between NHERF1 KO mouse plasma and WT alone was a decrease in the oxidized form of cysteine, CySS. CySS is necessary for the production of the cellular antioxidant GSH (161). Interestingly, the kidney GSH levels of NHERF1 KO mice do not reflect the decrease in plasma CySS. The decrease in CySSG and GSSG in the kidney after cisplatin administration are consistent with other reports, however GSH was, unexpectedly, not decreased in either genotype (159). Furthermore, the oxidized form of glutathione, GSSG, was increased in the kidneys of vehicle treated NHERF1 KO mice. In summary, NHERF1 KO mice had a decrease in oxidized cysteine in the plasma, an increase in oxidized glutathione in the kidney, and a decrease in kidney redox potential (GSH/GSSG) when compared to WT. These results may indicate that NHERF1 KO kidneys are under a level of oxidative stress and support the findings in Chapter III, where the pentose 
phosphate enzymes may be increased to compensate with a higher NADPH production to maintain cellular redox levels. On the other hand, NHERF1 KO kidneys do not have a decrease in GSH levels and appear to not have changes in GSH metabolism itself.

Lastly, enzymes thought to be involved in cisplatin's mechanism to a reactive thiol were considered. GGT activity in the urine was found to be highest between 4 and 24 hours following cisplatin treatment in humans (154), indicating these time points should be used in assessing differences in activity level. At 4 hours GGT activity remained unvarying between the treatment groups. However, at 24 hours WT mouse kidneys responded to cisplatin treatment with a substantial increase in activity while NHERF1 KO kidneys maintained a steady activity level. These data indicate that NHERF1 KO mice respond differently to the cisplatin insult. Whether this response is time dependent or based on functional ability of the enzyme is unclear. A 12 hour time point may be helpful to further elucidate the changes in GGT activity in treated NHERF1 KO mice. Interestingly, GGT protein levels were unchanged between the treatment groups via western blot. IHC was also performed on NHERF1 KO and WT kidneys resulting in similar staining intensity and localization of GGT within the tubules under baseline conditions. However, 24 hours after cisplatin treatment NHERF1 KO kidneys began to show some histologic changes including BBM sloughing which coincided with decreased staining for GGT. These data are consistent with other studies performed on rats at 72 hours, where increased damage to the proximal tubule coincided with decreased GGT expression and activity $(116,154,163)$. 
Although NHERF1 KO kidney GGT protein expression remained insignificant with cisplatin treatment it is apparent that the degree of injury at 24 hours is less than what is seen at 72 hours. It is possible that NHERF1 KO mouse kidneys are beginning to lose GGT at 24 hours through BBM sloughing, but have not lost a significant amount of GGT protein at this time point. These experiments could be repeated on 72 hour cisplatin treated mice for improved interpretation of these results. CCBL protein was also measured in these mice. Similar to the GGT western blot data CCBL protein remained insignificantly altered between the different groups.

This study has provided some clarification regarding changes in the renal handling of cisplatin with NHERF1 loss. The main finding being an altered response in GGT activity with the cisplatin insult. Furthermore, this study confirmed that NHERF1 KO mice are indeed more sensitive to the cisplatin insult. NHERF1 KO mice do not have overt signs of oxidative stress, but this may be masked by compensatory mechanisms in the pentose phosphate pathway. Most importantly, this study has laid the groundwork for future experiments regarding altered renal handling of cisplatin in the NHERF1 KO mouse. A few examples include: implementing a 12 hour time point to study time dependent changes in GGT activity following cisplatin treatment, CCBL activity and localization, and evaluating GGT and CCBL localization and protein levels 72 hours after cisplatin treatment.

\section{Significance of new findings}


Chapter III investigated metabolic changes in NHERF1 KO mice that may predispose these animals to cisplatin nephrotoxicity. Moreover, gluconeogenic and glycolytic enzymes were found to be unchanged, but ME and G6PD had increased activities with NHERF1 loss. The significance of these findings is that that NHERF1 KO mice may have compensatory mechanisms in the NADPHdependent processes for maintenance of the cellular redox state. G6PD and ME are the two main sources of cytosolic NADPH, which is important for protection from oxidative stress (166). Furthermore, G6PD activity through the generation of ribose-5-phosphate is also involved in nucleic acid synthesis and repair (164). Therefore, enhancement of ME and G6PD is consistent with the presence of some underlying stress in the NHERF1 KO mouse. The combined effect of an underlying oxidative stress and the cisplatin-induced oxidative stress could result in increased sensitivity and exacerbation of injury with NHERF1 loss.

The work in Chapter IV involved characterizing differences in mitochondrial structure and/or function between WT and NHERF1 KO kidneys. Interestingly, no changes in mitochondrial structure, number or size were found to indicate an altered function or mitochondrial oxidative stress. Furthermore, Seahorse XF24 functional assays determined that isolated NHERF1 mitochondria function normally when compared to WT. This observation suggests that NHERF1 KO mitochondria have normal bioenergetics when all required compounds are available. However, NHERF1 KO mice do undergo phosphate wasting (148), potentially creating a suboptimal intracellular environment for mitochondria. Inorganic phosphate is essential for many cellular functions such as signal 
transduction and energy metabolism. For instance, inorganic phosphate uptake into mitochondria is required for oxidative phosphorylation and the subsequent production of ATP (167). Mitochondrial inorganic phosphate also activates matrix enzymes and ETC activity (167). Moreover, in insulin secreting cells inorganic phosphate uptake results in further hyperpolarization of the electrical gradient (167). Therefore, the significance of this work is the formulation of a novel potential mechanism of susceptibility to cisplatin-induced AKI. Thus, NHERF1 KO mice may have mitochondrial dysfunction due to phosphate wasting that sensitizes the kidney to cisplatin toxicity. Seahorse XF24 functional analysis on whole kidney tissue would be beneficial for future studies.

The biotransformation of cisplatin to a nephrotoxin is poorly understood and the state of research in the field is encumbered with controversial results. Therefore, more research is needed in this area for the development of future therapies or early prognostic tests. In Chapter $\mathrm{V}$ changes in the renal handling of cisplatin were investigated in the NHERF1 KO mouse. First, NHERF1 KO mice were confirmed to have increased sensitivity to the initial cisplatin insult. This was demonstrated by early histologic signs of damage in the NHERF1 KO kidney when compared to WT. Additionally, urine NGAL was significantly increased in the 24 hour cisplatin treated NHERF1 KO mouse in comparison to the WT. Following that information, lipid peroxidation levels were assessed and found to be minimal in vehicle treated WT and NHERF1 KO mice. However, the cisplatin treated NHERF1 KO mouse had increased 4-HNE staining in the cortex and JM regions when compared to WT. Reduced and oxidized forms of Cys and GSH 
were compared between WT and NHERF1 KO mice. Although there were some differences in vehicle treated NHERF1 KO mice there were no overt signs of oxidative stress. GSH levels remained unchanged in both WT and NHERF1 KO animals, however, the kidney redox potential in the NHERF1 KO mouse was significantly decreased when compared to WT. An increase in redox potential is indicative of increased oxidative stress. However, with the increased activity in G6PD and ME found in Chapter III, indicators of oxidative stress may be masked. Thus, a decrease in redox potential may be a sign of compensatory mechanisms to protect from oxidative stress. Finally, since GSH levels were unchanged between WT and NHERF1 KO mice it appears the KO mouse has unaltered GSH metabolism. In addition to the thiol measurements GGT activity, protein level, and localization was also assessed. While the protein level was unchanged between all treatment groups the GGT activity response was found to be altered with cisplatin treatment in the KO mouse. GGT is important for detoxification of xenobiotics and production of GSH, and thus, increased activity may alleviate some of the induced oxidative stress from cisplatin. If an increase in GGT activity is protective then the NHERF1 KO may not have the ability to increase GGT activity quickly and/or robustly with the cisplatin insult, resulting in exacerbated injury. Alternatively, if an increase in GGT activity is detrimental, then a 12 hour time point may be useful in determining if GGT activity fluctuates over time. Therefore, since the KO mouse already has kidney damage at 24 hours there could be an increase in activity between 4 and 24 hours resulting in this early injury. Another potential explanation for a lack of increase in GGT activity may be 
due to the histologic changes seen 24 hours after cisplatin treatment, where NHERF1 KO mice have patchy areas of BBM sloughing resulting in GGT loss. This hypothesis would coincide with data from other studies using 72 hour cisplatin treated rats. When the rats exhibited increased damage to the proximal tubule the damage corresponded with decreased GGT expression and activity $(116,154,163)$. Therefore, it is possible that NHERF1 KO mice are beginning to lose GGT at 24 hours through BBM sloughing, but have not lost a significant amount of GGT protein to be reflected in the western blot data. Finally, CCBL protein was also considered in this study. Similar to the GGT protein there were no significant changes in CCBL between any of the treatment groups. These data provide insight into the effects of NHERF1 loss and renal handling of cisplatin. Furthermore, this insight has contributed to upcoming experiments that may lead to new hypotheses and therapeutic approaches in the study of cisplatin-induced AKI.

\section{Strengths and weaknesses of this dissertation}

\section{Strengths}

There are several strengths of this dissertation. The first study provides insight into the role of NHERF1 loss and metabolic changes in the pentose phosphate pathway (Chapter III). The second study (Chapter IV) established that NHERF1 KO mice isolated mitochondria do not have structural or functional changes that would result in dysfunction. Lastly, Chapter V established that NHERF1 KO mice are indeed sensitive to the initial cisplatin insult and have an 
altered GGT activity response when given cisplatin. Overall this dissertation identifies that NHERF1 KO mice respond differently to the cisplatin insult. Additionally, it provides novel hypotheses that could lead to potential therapeutic approaches or prognostic tests for cisplatin-induced AKI. Currently, no FDA approved therapy exists to halt or reverse cisplatin-induced AKI.

All the work in Chapters III and V was performed in the whole animal. Use of the whole animal is particularly important when studying diseases and allows better translation to human disease. Chapter IV utilized sensitive approaches in establishing mitochondrial changes in the NHERF1 KO mouse. Additionally, these studies used multiple time points with an established acute model of cisplatin-induced AKI. Furthermore, pharmaceutical grade cisplatin was used when treating the animals. While no model fully recapitulates the human disease, this model provided an established result of nephrotoxicity in WT C57BL/6J mice. Thus, the experimenter can determine changes in response to cisplatin in the NHERF1 KO mice. Additionally, the mice used in these experiments were all within the same age range (2-4 months old) and were littermates. These steps were taken in order to ensure the animals used were young, healthy, and did not have genetic drift that may affect the results of these studies.

The renal circadian clock is known to regulate the dosing-time dependency of cisplatin-induced nephrotoxicity in mice (168). All of the mice used in this study were treated with cisplatin at approximately 10 a.m. and sacrificed either 24 or 72 hours later. Unfortunately, the 4 hour time point was conducted earlier at 6 a.m. due to restrictions in access to the mice. However, it was deemed to be more 
important to sacrifice all the animals at the same time point of 10 a.m. This decision was made after extensive literature searches, where other studies found that when mice were treated with cisplatin later in the day they exhibited less kidney injury (169). Thus, this experimental design attempts to mitigate any effects of circadian rhythms on cisplatin nephrotoxicity.

Experimental design is extremely important in mitigating factors that could influence results. In addition to using established animal models of cisplatininduced $\mathrm{AKI}$ and taking into account circadian rhythm, most of these studies used five or more animals in each treatment group. This number is due in part to the increased sensitivity of the NHERF1 KO mouse to cisplatin and the variability in kidney injury seen with cisplatin treatment. As previously mentioned these studies also utilized highly sensitive methods for analysis. For instance, LC-MS was utilized in order to determine the ATP content of WT and NHERF1 KO kidneys. This method is very sensitive and is advantageous when compared to commercially available kits used to measure ATP.

\section{Weaknesses}

The main purpose of this work was to investigate potential mechanisms of susceptibility of the NHERF1 KO mouse to cisplatin nephrotoxicity. In order to study the effects NHERF1 loss and its effect before and after cisplatin treatment, WT C57BL/6J and C57BL/6J mice with a global deletion of NHERF1 were used. Whole mouse deletions can result in non-specific effects due to compensatory mechanisms that are selected for early in development. NHERF1 KO mice are 
viable, fertile, and histologically the kidneys are similar to WT. However, NHERF1 KO mice do have alterations in kidney function, phosphate wasting, uric acid wasting, and hypercalciuria (46). The potential roles of alterations in electrolyte metabolism were not tested in this study.

Although there are strengths associated with the use of a mouse model, there are also limitations. The use of in vivo experiments may recapitulate human disease more completely when compared to in vitro experiments. Unfortunately, the use of in vivo research also involves less control of variables and introduces complexities. Furthermore, relevance to human disease may be questionable when mouse models are compared to human studies. At this time the role of NHERF1 loss and susceptibility to cisplatin nephrotoxicity has not been studied in humans. It is therefore possible that NHERF1 loss is not detrimental or that the observed changes in the pentose phosphate pathway and in GGT activity may be mouse-specific and have little relevance to humans. While these aspects of in vivo models can never be entirely avoided, it can be optimized for the question at hand and taken into consideration when interpreting results.

Other limitations of this study involve the ambiguous and complex process of cisplatin biotransformation in the kidney. The controversial role of GGT activity has been extensively discussed in this dissertation. However, another limitation in studying cisplatin metabolism involves CCBL. Mammals have at least 10 variations in CCBL, none of which have been conclusively linked with cisplatin nephrotoxicity (170). However, glutamine transaminase K (GTK) has a high inherent CCBL activity and therefore is the most studied in cisplatin 
nephrotoxicity. In this dissertation the antibody against CCBL1 or GTK was utilized and future activity assays will also be performed using GTK. This added complication is another limitation when studying the biotransformation of cisplatin. Therefore, any results using GTK may not be relevant in mice let alone human subjects.

\section{E. Future Directions}

While the experiments described in this dissertation answered specific gaps in our knowledge of NHERF1 loss and susceptibility to cisplatin nephrotoxicity, it has also created new questions that will need to be addressed in future studies. Seven of these questions are discussed below.

\section{Is the increased ME and G6PD activity identified in Chapter III a compensatory mechanism to maintain the cellular redox state in NHERF1 KO mice?}

In Chapter III ME and G6PD activity was increased in the NHERF1 KO mouse and suggested the possibility of increased NADPH production to provide protection from oxidative stress. Therefore, measuring NADPH levels in the WT and NHERF1 KO kidneys is necessary to answer this question. To carry out this investigation LC-MS analysis would be the most sensitive method in measuring NADPH levels. Additionally, ATP and NADPH could be measured in cisplatin treated animals to further understand the bioenergetics of the NHERF1 KO mouse in response to insult. In the previous study cisplatin treated NHERF1 KO 
mice were found to have increased ATN (35), which may be indicative of decreased ATP and/or NADPH levels. In addition, measuring gene and protein expression of ME and G6PD could also be investigated to further understand any compensatory mechanisms of the NHERF1 KO mouse.

\section{Do the NHERF1 KO mitochondria function normally in whole tissue or with changes in substrate?}

In Chapter IV NHERF1 KO kidney mitochondria were found to function normally in optimal conditions, however this does not answer how the mitochondria function in their native environment. Fortunately, Agilent has developed Seahorse XF24 islet capture microplates which can be used to analyze whole tissue. Furthermore, there have been previous studies using this method in multiple tissue types (171-173). Optimization will be needed for the kidney, but whole tissue analysis will be useful in determining how NHERF1 KO mitochondria function within the kidney. In addition to examining mitochondrial function in the kidney, other substrates could be used besides succinate/rotenone when assessing isolated mitochondrial function. Agilent also has a palmitate-BSA fatty acid oxidation substrate that can be used to assess fatty acid oxidation in isolated mitochondria. This consideration is of importance as proximal renal tubules use fatty acids as their major source of energy.

\section{Does cisplatin damage NHERF1 KO mitochondria to a greater degree than WT mitochondria?}


Cisplatin has been established in inducing ROS and mitochondrial dysfunction $(139,140,142,143)$. Furthermore, platinum can be found in mitochondria 24 hours after cisplatin treatment. This information combined with studies from Chapter IV gives rise to multiple future experiments. WT and NHERF1 KO mice could be treated with cisplatin for 24 and 72 hours and mitochondria can be isolated from these kidneys for Seahorse XF24 functional analysis and measurement of platinum by inductively coupled plasma-mass spectrometry (ICP-MS). Additionally, ROS could be measured in isolated mitochondria using mitoSOX for mitochondrial $\mathrm{O}_{2}$ detection. These studies could help in understanding if cisplatin is taken up to a greater degree in NHERF1 KO mitochondria and if cisplatin damages these mitochondria to a greater degree resulting in enhanced injury.

\section{Does NHERF1 loss result in altered renal handling of cisplatin besides GGT activity?}

In addition to GGT activity CCBL activity can also be measured between vehicle and cisplatin treated WT and NHERF1 KO mice. There are multiple methods to assess CCBL activity, however GTK activity assay is simple and has been well studied (174). Additionally, IHC can be utilized to assess CCBL localization in vehicle and cisplatin treated WT and NHERF1 KO kidneys. ICPMS can also be used to measure platinum levels between cisplatin treated WT and NHERF1 KO kidneys. An increase in platinum in the NHERF1 KO kidney could indicate altered uptake or extrusion of cisplatin. Higher concentrations of 
cisplatin have been associated with increased necrotic cell death which was seen in the 72 hour cisplatin treated NHERF1 KO mouse (35). Furthermore, WT and NHERF1 KO mice could be treated with known inhibitors for OCT2, MATE1, and GGT in order to determine if the KO mouse responds the same and exhibits either amelioration or enhancement of toxicity. These outcomes could help to identify key players in the susceptibility of the NHERF1 KO mouse to cisplatin nephrotoxicity. Lastly, OK and NHERF1 deficient OK proximal tubule cells could be used to overexpress GGT to evaluate the effect of increased GGT expression on activity after treatment with cisplatin. This information would again, help to elucidate if increased GGT activity is detrimental or protective with NHERF1 loss and cisplatin treatment.

\section{How do NHERF1 KO mice respond to a chronic model of cisplatin nephrotoxicity?}

Utilization of a chronic repeated dosing model of cisplatin nephrotoxicity could help in determining if NHERF1 KO mice are only sensitive to an acute high dose of cisplatin or if they are equally sensitive to lower repeated doses over time. In WT mice the kidney damage associated with the chronic model is less severe and results in longer survival (175). Studying NHERF1 loss and cisplatin nephrotoxicity in a chronic model could provide information in understanding conditions when NHERF1 loss is detrimental with cisplatin administration. 


\section{Do heterozygous NHERF1 KO mice have some protection to cisplatin}

nephrotoxicity?

All of the studies mentioned in this dissertation have been conducted with complete loss of the NHERF1 protein. To broaden our knowledge of susceptibility to cisplatin nephrotoxicity with NHERF1 loss, heterozygous mice could also be tested using the acute cisplatin model. This could help answer the following questions: [1] is complete loss of NHERF1 expression needed for susceptibility to cisplatin nephrotoxicity and [2] is there a percent loss of NHERF1 that predisposes these animals to cisplatin nephrotoxicity?

\section{Does NHERF1 loss predispose mouse kidneys to other nephrotoxins or forms of AKI?}

These studies have only evaluated the nephrotoxic effects of cisplatin in NHERF1 KO mice. It would be interesting to establish if these mice are only sensitive to cisplatin or if other nephrotoxins also result in enhanced injury. For example, cyclosporine, vancomycin, acyclovir, lead, mercury, and cadmium could be tested. In addition, models of ischemic reperfusion could also be evaluated.

\section{F. Summary and conclusions}

The overall goal of the work described in this dissertation was to discover new potential mechanisms and hypotheses for susceptibility to cisplatin-induced AKI with NHERF1 loss. Chapter III evaluated metabolic alterations in the NHERF1 
KO mouse that may play a role in the susceptibility to cisplatin insult. This study provided the foundation for new hypotheses and future experiments concerning changes in NADPH production in the NHERF1 KO mouse and masked signs of oxidative stress that may predispose these animals to injury. Chapter IV aimed to evaluate if NHERF1 KO mice had an underlying mitochondrial dysfunction due to: [1] decreases in mitochondrial oxidative capacity and/or alterations in the structure of NHERF1 KO kidneys [2] decreases in number of mitochondria in NHERF1 KO kidney. This study did not find any evidence of mitochondrial dysfunction in the NHERF1 KO mouse. Finally, Chapter V assessed if NHERF1 loss resulted in altered renal handling of cisplatin and found an altered GGT activity response when NHERF1 KO mice were given cisplatin. Taken together, these studies provide new insight into the mechanism of susceptibility of NHERF1 loss in cisplatin-induced AKI. 


\section{REFERENCES}

1. Boron WF, Boulpaep EL. Medical physiology : a cellular and molecular approach. Updated ed. Philadelphia, Pa.: Elsevier Saunders; 2005. xiii, 1319 p.

p.

2. Yu AS. Brenner \& rector's the kidney. 11th edition. ed. pages $\mathrm{cm} \mathrm{p}$.

3. Eaton DC, Pooler J. Vander's renal physiology. Eighth edition. ed. vii, 204 pages $p$.

4. Connell A, Laing C. Acute kidney injury. Clinical medicine (London, England). 2015;15(6):581-4. doi: 10.7861/clinmedicine.15-6-581.

5. Davis ENMHSPAC. Acute Kidney Injury : The Ugly Truth. Kidney Disease. 2016;1(1):149-59. doi: 10.1016/j.cpha.2015.09.006.

6. Kane-Gill SL, Goldstein SL. Drug-Induced Acute Kidney Injury. Critical Care Clinics. 2015;31(4):675-84. doi: 10.1016/j.ccc.2015.06.005.

7. Schetz M, Dasta J, Goldstein S, Golper T. Drug-induced acute kidney injury. Current opinion in critical care. 2005;11(6):555-65.

8. Hanigan $\mathrm{MH}$, Devarajan P. Cisplatin nephrotoxicity: molecular mechanisms. Cancer therapy. 2003;1:47-61. PubMed PMID: 18185852; PubMed Central PMCID: PMC2180401.

9. Eustace P. History and development of cisplatin in the management of malignant disease. Cancer nursing. 1980;3(5):373-8.

10. Alderden RA, Hall MD, Hambley TW. The Discovery and Development of Cisplatin. Journal of Chemical Education. 2006;83(5):728. doi:

10.1021/ed083p728.

11. (NCl) NCl. Cisplatin2017.

12. Pabla N, Dong Z. Cisplatin nephrotoxicity: mechanisms and renoprotective strategies. Kidney international. 2008;73(9):994-1007. doi:

10.1038/sj.ki.5002786. PubMed PMID: 18272962.

13. Miller RP, Tadagavadi RK, Ramesh G, Reeves WB. Mechanisms of Cisplatin nephrotoxicity. Toxins. 2010;2(11):2490-518. doi:

10.3390/toxins2112490. PubMed PMID: 22069563; PubMed Central PMCID: PMC3153174.

14. DeConti RC, Toftness BR, Lange RC, Creasey WA. Clinical and pharmacological studies with cis-diamminedichloroplatinum (II). Cancer research. 1973;33(6):1310-5. PubMed PMID: 4515709.

15. Cornelison TL, Reed E. Nephrotoxicity and hydration management for cisplatin, carboplatin, and ormaplatin. Gynecologic oncology. 1993;50(2):147-58. doi: 10.1006/gyno.1993.1184. PubMed PMID: 8375728. 
16. Safirstein R, Miller P, Guttenplan JB. Uptake and metabolism of cisplatin by rat kidney. Kidney international. 1984;25(5):753-8. doi: 10.1038/ki.1984.86. PubMed PMID: 6540826.

17. Kolb RJ, Ghazi AM, Barfuss DW. Inhibition of basolateral transport and cellular accumulation of CDDP and N-acetyl- L-cysteine-cDDP by TEA and PAH in the renal proximal tubule. Cancer chemotherapy and pharmacology. 2003;51(2):132-8. doi: 10.1007/s00280-002-0537-0. PubMed PMID: 12647014. 18. Pabla N, Murphy RF, Liu K, Dong Z. The copper transporter Ctr1 contributes to cisplatin uptake by renal tubular cells during cisplatin nephrotoxicity. American journal of physiology Renal physiology. 2009;296(3):F505-11. doi: 10.1152/ajprenal.90545.2008. PubMed PMID: 19144690; PubMed Central PMCID: PMC2660190.

19. Ciarimboli G, Deuster D, Knief A, Sperling M, Holtkamp M, Edemir B, Pavenstadt $H$, Lanvers-Kaminsky C, am Zehnhoff-Dinnesen A, Schinkel AH, Koepsell H, Jurgens $H$, Schlatter E. Organic cation transporter 2 mediates cisplatin-induced oto- and nephrotoxicity and is a target for protective interventions. The American journal of pathology. 2010;176(3):1169-80. doi: 10.2353/ajpath.2010.090610. PubMed PMID: 20110413; PubMed Central PMCID: PMC2832140.

20. Filipski KK, Mathijssen RH, Mikkelsen TS, Schinkel AH, Sparreboom A. Contribution of organic cation transporter 2 (OCT2) to cisplatin-induced nephrotoxicity. Clinical pharmacology and therapeutics. 2009;86(4):396-402. doi: 10.1038/clpt.2009.139. PubMed PMID: 19625999; PubMed Central PMCID: PMC2746866.

21. Ludwig T, Riethmuller C, Gekle M, Schwerdt G, Oberleithner H. Nephrotoxicity of platinum complexes is related to basolateral organic cation transport. Kidney international. 2004;66(1):196-202. doi: 10.1111/j.15231755.2004.00720.x. PubMed PMID: 15200426.

22. Nakamura T, Yonezawa A, Hashimoto S, Katsura T, Inui K. Disruption of multidrug and toxin extrusion MATE1 potentiates cisplatin-induced nephrotoxicity. Biochemical pharmacology. 2010;80(11):1762-7. doi: 10.1016/j.bcp.2010.08.019. PubMed PMID: 20813096.

23. Townsend DM, Tew KD, He L, King JB, Hanigan MH. Role of glutathione $\mathrm{S}$-transferase $\mathrm{Pi}$ in cisplatin-induced nephrotoxicity. Biomedicine \& pharmacotherapy = Biomedecine \& pharmacotherapie. 2009;63(2):79-85. doi: 10.1016/j.biopha.2008.08.004. PubMed PMID: 18819770; PubMed Central PMCID: PMC2667699.

24. Sadzuka Y, Shimizu Y, Takino Y, Hirota S. Protection against cisplatininduced nephrotoxicity in the rat by inducers and an inhibitor of glutathione Stransferase. Biochemical pharmacology. 1994;48(3):453-9. doi: 10.1016/00062952(94)90274-7. PubMed PMID: 8068032.

25. Townsend DM, Deng M, Zhang L, Lapus MG, Hanigan MH. Metabolism of Cisplatin to a nephrotoxin in proximal tubule cells. Journal of the American Society of Nephrology : JASN. 2003;14(1):1-10. doi:

10.1097/01.asn.0000042803.28024.92. PubMed PMID: 12506132; PubMed Central PMCID: PMC6361148. 
26. Townsend $\mathrm{DM}$, Hanigan $\mathrm{MH}$. Inhibition of gamma-glutamyl transpeptidase or cysteine S-conjugate beta-lyase activity blocks the nephrotoxicity of cisplatin in mice. The Journal of pharmacology and experimental therapeutics.

2002;300(1):142-8. doi: 10.1124/jpet.300.1.142. PubMed PMID: 11752109;

PubMed Central PMCID: PMC6522257.

27. Zhang L, Hanigan $\mathrm{MH}$. Role of cysteine S-conjugate beta-lyase in the metabolism of cisplatin. The Journal of pharmacology and experimental therapeutics. 2003;306(3):988-94. doi: 10.1124/jpet.103.052225. PubMed PMID: 12750429.

28. Lieberthal W, Triaca V, Levine J. Mechanisms of death induced by cisplatin in proximal tubular epithelial cells: apoptosis vs. necrosis. The American journal of physiology. 1996;270(4 Pt 2):F700-8. doi:

10.1152/ajprenal.1996.270.4.F700. PubMed PMID: 8967349.

29. Liu H, Baliga R. Cytochrome P450 2E1 null mice provide novel protection against cisplatin-induced nephrotoxicity and apoptosis. Kidney international. 2003;63(5):1687-96. doi: 10.1046/j.1523-1755.2003.00908.x. PubMed PMID: 12675844.

30. Weinman EJ, Steplock D, Corry D, Shenolikar S. Identification of the human NHE-1 form of $\mathrm{Na}(+)-\mathrm{H}+$ exchanger in rabbit renal brush border membranes. The Journal of clinical investigation. 1993;91(5):2097-102. doi: 10.1172/JCl116433. PubMed PMID: 8486777; PubMed Central PMCID: PMC288209.

31. Khundmiri SJ, Weinman EJ, Steplock D, Cole J, Ahmad A, Baumann PD, Barati M, Rane MJ, Lederer E. Parathyroid hormone regulation of $\mathrm{NA+,K+-}$ ATPase requires the PDZ 1 domain of sodium hydrogen exchanger regulatory factor-1 in opossum kidney cells. Journal of the American Society of Nephrology : JASN. 2005;16(9):2598-607. doi: 10.1681/ASN.2004121049. PubMed PMID: 16000700.

32. Cheng H, Li J, Fazlieva R, Dai Z, Bu Z, Roder H. Autoinhibitory interactions between the PDZ2 and C-terminal domains in the scaffolding protein NHERF1. Structure. 2009;17(5):660-9. doi: 10.1016/j.str.2009.03.009. PubMed PMID: 19446522; PubMed Central PMCID: PMC2688836.

33. Dai JL, Wang L, Sahin AA, Broemeling LD, Schutte M, Pan Y. NHERF $(\mathrm{Na}+/ \mathrm{H}+$ exchanger regulatory factor) gene mutations in human breast cancer. Oncogene. 2004;23(53):8681-7. doi: 10.1038/sj.onc.1207962. PubMed PMID: 15467753.

34. Georgescu MM, Morales FC, Molina JR, Hayashi Y. Roles of NHERF1/EBP50 in cancer. Current molecular medicine. 2008;8(6):459-68. PubMed PMID: 18781953.

35. Bushau-Sprinkle A, Barati M, Conklin C, Dupre T, Gagnon KB, Khundmiri SJ, Clark B, Siskind L, Doll MA, Rane M, Brier M, Coventry S, Lederer ED. Loss of the $\mathrm{Na}(+) / \mathrm{H}(+)$ Exchange Regulatory Factor 1 Increases Susceptibility to Cisplatin-Induced Acute Kidney Injury. The American journal of pathology. 2019;189(6):1190-200. doi: 10.1016/j. ajpath.2019.02.010. PubMed PMID: 30926337; PubMed Central PMCID: PMC6547057. 
36. Li M, Mennone A, Soroka CJ, Hagey LR, Ouyang X, Weinman EJ, Boyer $\mathrm{JL}$. $\mathrm{Na}(+) / H(+)$ exchanger regulatory factor 1 knockout mice have an attenuated hepatic inflammatory response and are protected from cholestatic liver injury. Hepatology. 2015;62(4):1227-36. doi: 10.1002/hep.27956. PubMed PMID: 26108984; PubMed Central PMCID: PMC4589453.

37. Leslie KL, Song GJ, Barrick S, Wehbi VL, Vilardaga JP, Bauer PM, Bisello A. Ezrin-radixin-moesin-binding phosphoprotein 50 (EBP50) and nuclear factorkappaB (NF-kappaB): a feed-forward loop for systemic and vascular inflammation. The Journal of biological chemistry. 2013;288(51):36426-36. doi: 10.1074/jbc.M113.483339. PubMed PMID: 24196963; PubMed Central PMCID: PMC3868756.

38. Sun L, Zheng J, Wang Q, Song R, Liu H, Meng R, Tao T, Si Y, Jiang W, He J. NHERF1 regulates actin cytoskeleton organization through modulation of alpha-actinin-4 stability. FASEB journal : official publication of the Federation of American Societies for Experimental Biology. 2016;30(2):578-89. doi: 10.1096/fj.15-275586. PubMed PMID: 26432781.

39. Bryant DM, Roignot J, Datta A, Overeem AW, Kim M, Yu W, Peng X, Eastburn DJ, Ewald AJ, Werb Z, Mostov KE. A molecular switch for the orientation of epithelial cell polarization. Developmental cell. 2014;31(2):171-87. doi: 10.1016/j.devcel.2014.08.027. PubMed PMID: 25307480; PubMed Central PMCID: PMC4248238.

40. Ketchem CJ, Khundmiri SJ, Gaweda AE, Murray R, Clark BJ, Weinman $\mathrm{EJ}$, Lederer ED. Role of $\mathrm{Na}+\mathrm{H}+$ exchanger regulatory factor 1 in forward trafficking of the type lla $\mathrm{Na}+$-Pi cotransporter. American journal of physiology Renal physiology. 2015;309(2):F109-19. doi: 10.1152/ajprenal.00133.2015. PubMed PMID: 25995109; PubMed Central PMCID: PMC4504931.

41. Schmieder S, Nagai M, Orlando RA, Takeda T, Farquhar MG.

Podocalyxin activates RhoA and induces actin reorganization through NHERF1 and Ezrin in MDCK cells. Journal of the American Society of Nephrology : JASN. 2004;15(9):2289-98. doi: 10.1097/01.ASN.0000135968.49899.E8. PubMed PMID: 15339978.

42. Favia M, Guerra L, Fanelli T, Cardone RA, Monterisi S, Di Sole F, Castellani S, Chen M, Seidler U, Reshkin SJ, Conese M, Casavola V. Na+/H+ exchanger regulatory factor 1 overexpression-dependent increase of cytoskeleton organization is fundamental in the rescue of F508del cystic fibrosis transmembrane conductance regulator in human airway CFBE410- cells. Molecular biology of the cell. 2010;21(1):73-86. doi: 10.1091/mbc.E09-03-0185. PubMed PMID: 19889841; PubMed Central PMCID: PMC2801722.

43. Jeong J, Kim W, Hens J, Dann P, Schedin P, Friedman PA, Wysolmerski JJ. NHERF1 Is Required for Localization of PMCA2 and Suppression of Early Involution in the Female Lactating Mammary Gland. Endocrinology. 2019;160(8):1797-810. doi: 10.1210/en.2019-00230. PubMed PMID: 31087002; PubMed Central PMCID: PMC6619491.

44. Antalffy G, Caride AJ, Paszty K, Hegedus L, Padanyi R, Strehler EE, Enyedi A. Apical localization of PMCA2w/b is enhanced in terminally polarized MDCK cells. Biochemical and biophysical research communications. 
2011;410(2):322-7. doi: 10.1016/j.bbrc.2011.05.147. PubMed PMID: 21672522; PubMed Central PMCID: PMC3139432.

45. Wang JQ, Qin F, Zhu L. Expression of $\mathrm{Na}+\mathrm{H}+$ exchanger regulatory factor 1 in autosomal-dominant polycystic kidney disease. The Journal of international medical research. 2015;43(5):629-38. doi:

10.1177/0300060515581182. PubMed PMID: 26142394.

46. Shenolikar S, Voltz JW, Minkoff CM, Wade JB, Weinman EJ. Targeted disruption of the mouse NHERF-1 gene promotes internalization of proximal tubule sodium-phosphate cotransporter type lla and renal phosphate wasting. Proc Natl Acad Sci U S A. 2002;99(17):11470-5. doi: 10.1073/pnas.162232699. PubMed PMID: 12169661; PubMed Central PMCID: PMCPMC123280.

47. Vaquero J, Nguyen Ho-Bouldoires TH, Claperon A, Fouassier L. Role of the PDZ-scaffold protein NHERF1/EBP50 in cancer biology: from signaling regulation to clinical relevance. Oncogene. 2017;36(22):3067-79. doi: 10.1038/onc.2016.462. PubMed PMID: 28068322.

48. Fang $X Y$, Song $R$, Chen $W$, Yang $Y Y, G u Y H$, Shu $Y Q, W u X D, W u X F$, Sun Y, Shen Y, Xu Q. PRL-3 Promotes the Malignant Progression of Melanoma via Triggering Dephosphorylation and Cytoplasmic Localization of NHERF1. The Journal of investigative dermatology. 2015;135(9):2273-82. doi:

10.1038/jid.2015.154. PubMed PMID: 25897829.

49. Cardone RA, Bellizzi A, Busco G, Weinman EJ, Dell'Aquila ME, Casavola V, Azzariti A, Mangia A, Paradiso A, Reshkin SJ. The NHERF1 PDZ2 domain regulates PKA-RhoA-p38-mediated NHE1 activation and invasion in breast tumor cells. Molecular biology of the cell. 2007;18(5):1768-80. doi: 10.1091/mbc.e0607-0617. PubMed PMID: 17332506; PubMed Central PMCID: PMC1855021. 50. Karn T, Ruckhaberle E, Hanker L, Muller V, Schmidt M, Solbach C, Gatje R, Gehrmann M, Holtrich U, Kaufmann M, Rody A. Gene expression profiling of luminal $B$ breast cancers reveals NHERF1 as a new marker of endocrine resistance. Breast cancer research and treatment. 2011;130(2):409-20. doi: 10.1007/s10549-010-1333-x. PubMed PMID: 21203899.

51. Song J, Bai J, Yang W, Gabrielson EW, Chan DW, Zhang Z. Expression and clinicopathological significance of oestrogen-responsive ezrin-radixinmoesin-binding phosphoprotein 50 in breast cancer. Histopathology. 2007;51(1):40-53. doi: 10.1111/j.1365-2559.2007.02730.x. PubMed PMID: 17593079 .

52. Malfettone A, Saponaro C, Paradiso A, Simone G, Mangia A. Peritumoral vascular invasion and NHERF1 expression define an immunophenotype of grade 2 invasive breast cancer associated with poor prognosis. BMC cancer. 2012;12:106. doi: 10.1186/1471-2407-12-106. PubMed PMID: 22439624; PubMed Central PMCID: PMC3362775.

53. Mangia A, Chiriatti A, Bellizzi A, Malfettone A, Stea B, Zito FA, Reshkin SJ, Simone G, Paradiso A. Biological role of NHERF1 protein expression in breast cancer. Histopathology. 2009;55(5):600-8. doi: 10.1111/j.13652559.2009.03424.x. PubMed PMID: 19912366.

54. Bellizzi A, Mangia A, Malfettone A, Cardone RA, Simone G, Reshkin SJ, Paradiso $\mathrm{A}$. $\mathrm{Na}+\mathrm{H}+$ exchanger regulatory factor 1 expression levels in blood and 
tissue predict breast tumour clinical behaviour. Histopathology. 2011;58(7):108695. doi: 10.1111/j.1365-2559.2011.03844.x. PubMed PMID: 21707710.

55. Tabrizi AD, Kalloger SE, Kobel M, Cipollone J, Roskelley CD, Mehl E, Gilks CB. Primary ovarian mucinous carcinoma of intestinal type: significance of pattern of invasion and immunohistochemical expression profile in a series of 31 cases. International journal of gynecological pathology : official journal of the International Society of Gynecological Pathologists. 2010;29(2):99-107. doi: 10.1097/PGP.0b013e3181bbbcc1. PubMed PMID: 20173494.

56. Accardi R, Rubino R, Scalise M, Gheit T, Shahzad N, Thomas M, Banks L, Indiveri C, Sylla BS, Cardone RA, Reshkin SJ, Tommasino M. E6 and E7 from human papillomavirus type 16 cooperate to target the PDZ protein $\mathrm{Na} / \mathrm{H}$ exchange regulatory factor 1 . Journal of virology. 2011;85(16):8208-16. doi: 10.1128/JVI.00114-11. PubMed PMID: 21680517; PubMed Central PMCID: PMC3147992.

57. Kreimann EL, Ratajska M, Kuzniacka A, Demacopulo B, Stukan M, Limon $J$. A novel splicing mutation in the SLC9A3R1 gene in tumors from ovarian cancer patients. Oncology letters. 2015;10(6):3722-6. doi: 10.3892/ol.2015.3796. PubMed PMID: 26788197; PubMed Central PMCID: PMC4665402.

58. Bartholow TL, Becich MJ, Chandran UR, Parwani AV.

Immunohistochemical analysis of ezrin-radixin-moesin-binding phosphoprotein 50 in prostatic adenocarcinoma. BMC urology. 2011;11:12. doi: 10.1186/14712490-11-12. PubMed PMID: 21672215; PubMed Central PMCID: PMC3132203. 59. Wang L, Du YR, Ji MY, Wang W, Zhan N, Zhou QS, Dong WG. Reduced EBP50 expression or mis-localization of the EBP50 protein is associated with the malignant progression of esophageal squamous cell carcinoma. European review for medical and pharmacological sciences. 2014;18(24):3854-63. PubMed PMID: 25555876.

60. Mangia A, Caldarola L, Dell'Endice S, Scarpi E, Saragoni L, Monti M, Santini D, Brunetti O, Simone G, Silvestris N. The potential predictive role of nuclear NHERF1 expression in advanced gastric cancer patients treated with epirubicin/oxaliplatin/capecitabine first line chemotherapy. Cancer biology \& therapy. 2015;16(8):1140-7. doi: 10.1080/15384047.2015.1056414. PubMed PMID: 26126066; PubMed Central PMCID: PMC4622862.

61. Ji MY, Fan DK, Lv XG, Peng XL, Lei XF, Dong WG. The detection of EBP50 expression using quantum dot immunohistochemistry in pancreatic cancer tissue and down-regulated EBP50 effect on PC-2 cells. Journal of molecular histology. 2012;43(5):517-26. doi: 10.1007/s10735-012-9424-0. PubMed PMID: 22622406.

62. Malfettone A, Silvestris N, Paradiso A, Mattioli E, Simone G, Mangia A. Overexpression of nuclear NHERF1 in advanced colorectal cancer: association with hypoxic microenvironment and tumor invasive phenotype. Experimental and molecular pathology. 2012;92(3):296-303. doi: 10.1016/j.yexmp.2012.03.004. PubMed PMID: 22440733.

63. Schirosi L, Mazzotta A, Opinto G, Pinto R, Graziano G, Tommasi S, Fucci L, Simone G, Mangia A. beta-catenin interaction with NHERF1 and RASSF1A methylation in metastatic colorectal cancer patients. Oncotarget. 
2016;7(42):67841-50. doi: 10.18632/oncotarget.12280. PubMed PMID: 27765918; PubMed Central PMCID: PMC5356523.

64. Fouassier L, Duan CY, Feranchak AP, Yun CH, Sutherland E, Simon F, Fitz JG, Doctor RB. Ezrin-radixin-moesin-binding phosphoprotein 50 is expressed at the apical membrane of rat liver epithelia. Hepatology. 2001;33(1):166-76. doi: 10.1053/jhep.2001.21143. PubMed PMID: 11124833. 65. Shibata T, Chuma M, Kokubu A, Sakamoto M, Hirohashi S. EBP50, a beta-catenin-associating protein, enhances Wnt signaling and is over-expressed in hepatocellular carcinoma. Hepatology. 2003;38(1):178-86. doi: 10.1053/jhep.2003.50270. PubMed PMID: 12830000.

66. Nguyen Ho-Bouldoires TH, Claperon A, Mergey M, Wendum D, DesboisMouthon C, Tahraoui S, Fartoux L, Chettouh H, Merabtene F, Scatton O, Gaestel M, Praz F, Housset C, Fouassier L. Mitogen-activated protein kinase-activated protein kinase 2 mediates resistance to hydrogen peroxide-induced oxidative stress in human hepatobiliary cancer cells. Free radical biology \& medicine. 2015;89:34-46. doi: 10.1016/j.freeradbiomed.2015.07.011. PubMed PMID: 26169728.

67. Mangia A, Partipilo G, Schirosi L, Saponaro C, Galetta D, Catino A, Scattone A, Simone G. Fine Needle Aspiration Cytology: A Tool to Study NHERF1 Expression as a Potential Marker of Aggressiveness in Lung Cancer. Molecular biotechnology. 2015;57(6):549-57. doi: 10.1007/s12033-015-9848-3. PubMed PMID: 25744438.

68. Kislin KL, McDonough WS, Eschbacher JM, Armstrong BA, Berens ME. NHERF-1: modulator of glioblastoma cell migration and invasion. Neoplasia. 2009;11(4):377-87. doi: 10.1593/neo.81572. PubMed PMID: 19308292; PubMed Central PMCID: PMC2657821.

69. Molina JR, Morales FC, Hayashi Y, Aldape KD, Georgescu MM. Loss of PTEN binding adapter protein NHERF1 from plasma membrane in glioblastoma contributes to PTEN inactivation. Cancer research. 2010;70(17):6697-703. doi: 10.1158/0008-5472.CAN-10-1271. PubMed PMID: 20736378; PubMed Central PMCID: PMC2932801.

70. Molina JR, Agarwal NK, Morales FC, Hayashi Y, Aldape KD, Cote G, Georgescu MM. PTEN, NHERF1 and PHLPP form a tumor suppressor network that is disabled in glioblastoma. Oncogene. 2012;31(10):1264-74. doi: 10.1038/onc.2011.324. PubMed PMID: 21804599; PubMed Central PMCID: PMC3208076.

71. Georgescu MM, Yell P, Mobley BC, Shang P, Georgescu T, Wang SH, Canoll P, Hatanpaa KJ, White CL, 3rd, Raisanen JM. NHERF1/EBP50 is an organizer of polarity structures and a diagnostic marker in ependymoma. Acta neuropathologica communications. 2015;3:11. doi: 10.1186/s40478-015-0197-z. PubMed PMID: 25775275; PubMed Central PMCID: PMC4352254.

72. Du G, Hao C, Gu Y, Wang Z, Jiang WG, He J, Cheng S. A Novel NHERF1 Mutation in Human Breast Cancer Inactivates Inhibition by NHERF1 Protein in EGFR Signaling. Anticancer research. 2016;36(3):1165-73. PubMed PMID: 26977012. 
73. Saponaro C, Vagheggini A, Scarpi E, Centonze M, Catacchio I, Popescu O, Pastena MI, Giotta F, Silvestris N, Mangia A. NHERF1 and tumor microenvironment: a new scene in invasive breast carcinoma. Journal of experimental \& clinical cancer research : CR. 2018;37(1):96. doi:

10.1186/s13046-018-0766-7. PubMed PMID: 29716631; PubMed Central PMCID: PMC5930748.

74. Wang L, Qi Y, Xiong Y, Peng Z, Ma Q, Zhang Y, Song J, Zheng J. EzrinRadixin-Moesin Binding Phosphoprotein 50 (EBP50) Suppresses the Metastasis of Breast Cancer and HeLa Cells by Inhibiting Matrix Metalloproteinase-2 Activity. Anticancer research. 2017;37(8):4353-60. doi: 10.21873/anticanres.11829. PubMed PMID: 28739728.

75. Saponaro C, Sergio S, Coluccia A, De Luca M, La Regina G, Mologni L, Famiglini V, Naccarato V, Bonetti D, Gautier C, Gianni S, Vergara D, Salzet M, Fournier I, Bucci C, Silvestri R, Passerini CG, Maffia M, Coluccia AML. betacatenin knockdown promotes NHERF1-mediated survival of colorectal cancer cells: implications for a double-targeted therapy. Oncogene. 2018;37(24):330116. doi: 10.1038/s41388-018-0170-y. PubMed PMID: 29551770; PubMed Central PMCID: PMC6002344.

76. Sun C, Zheng J, Cheng S, Feng D, He J. EBP50 phosphorylation by Cdc2/Cyclin B kinase affects actin cytoskeleton reorganization and regulates functions of human breast cancer cell line MDA-MB-231. Molecules and cells. 2013;36(1):47-54. doi: 10.1007/s10059-013-0014-0. PubMed PMID: 23775624; PubMed Central PMCID: PMC3887931.

77. Song GJ, Leslie KL, Barrick S, Mamonova T, Fitzpatrick JM, Drombosky KW, Peyser N, Wang B, Pellegrini M, Bauer PM, Friedman PA, Mierke DF, Bisello A. Phosphorylation of ezrin-radixin-moesin-binding phosphoprotein 50 (EBP50) by Akt promotes stability and mitogenic function of S-phase kinaseassociated protein-2 (Skp2). The Journal of biological chemistry. 2015;290(5):2879-87. doi: 10.1074/jbc.M114.609768. PubMed PMID: 25492869; PubMed Central PMCID: PMC4317011.

78. Sun J, Li P, Yang J. Repressing of NHERF1 inhibits liver cancer progression by promoting the production of ROS. Biochemical and biophysical research communications. 2019;509(1):8-15. doi: 10.1016/j.bbrc.2018.11.121. PubMed PMID: 30581004.

79. Cardone RA, Greco MR, Capulli M, Weinman EJ, Busco G, Bellizzi A, Casavola V, Antelmi E, Ambruosi B, Dell'Aquila ME, Paradiso A, Teti A, Rucci N, Reshkin SJ. NHERF1 acts as a molecular switch to program metastatic behavior and organotropism via its PDZ domains. Molecular biology of the cell.

2012;23(11):2028-40. doi: 10.1091/mbc.E11-11-0911. PubMed PMID: 22496422; PubMed Central PMCID: PMC3364169.

80. Greco MR, Bon E, Rubino R, Guerra L, Bernabe-Garcia M, Cannone S, Cayuela ML, Ciaccia L, Marionneau-Lambot S, Oullier T, Fromont G, Guibon R, Roger S, Reshkin SJ, Cardone RA. Phosphorylation of NHERF1 S279 and S301 differentially regulates breast cancer cell phenotype and metastatic organotropism. Biochimica et biophysica acta Molecular basis of disease. 
2019;1865(1):26-37. doi: 10.1016/j.bbadis.2018.10.017. PubMed PMID: 30326259.

81. Al Ghouleh I, Meijles DN, Mutchler S, Zhang Q, Sahoo S, Gorelova A, Henrich Amaral J, Rodriguez AI, Mamonova T, Song GJ, Bisello A, Friedman PA, Cifuentes-Pagano ME, Pagano PJ. Binding of EBP50 to Nox organizing subunit p47phox is pivotal to cellular reactive species generation and altered vascular phenotype. Proceedings of the National Academy of Sciences of the United States of America. 2016;113(36):E5308-17. doi: 10.1073/pnas.1514161113. PubMed PMID: 27540115; PubMed Central PMCID: PMC5018796.

82. Jiang Y, Lu G, Trescott LR, Hou Y, Guan X, Wang S, Stamenkovich A, Brunzelle J, Sirinupong N, Li C, Yang Z. New conformational state of NHERF1CXCR2 signaling complex captured by crystal lattice trapping. PloS one. 2013;8(12):e81904. doi: 10.1371/journal.pone.0081904. PubMed PMID: 24339979; PubMed Central PMCID: PMC3858284.

83. Katoh M. Multilayered prevention and treatment of chronic inflammation, organ fibrosis and cancer associated with canonical WNT/betacatenin signaling activation (Review). International journal of molecular medicine. 2018;42(2):71325. doi: 10.3892/ijmm.2018.3689. PubMed PMID: 29786110; PubMed Central PMCID: PMC6034925.

84. Barati MT, Ketchem CJ, Merchant ML, Kusiak WB, Jose PA, Weinman EJ, LeBlanc AJ, Lederer ED, Khundmiri SJ. Loss of NHERF-1 expression prevents dopamine-mediated Na-K-ATPase regulation in renal proximal tubule cells from rat models of hypertension: aged F344 rats and spontaneously hypertensive rats. American journal of physiology Cell physiology. 2017;313(2):C197-C206. doi: 10.1152/ajpcell.00219.2016. PubMed PMID: 28515088; PubMed Central PMCID: PMC5582877.

85. Yang F, Gu Y, Zhao Z, Huang J, Jiang WG, Cheng S. NHERF1 Suppresses Lung Cancer Cell Migration by Regulation of EpithelialMesenchymal Transition. Anticancer research. 2017;37(8):4405-14. doi: 10.21873/anticanres.11835. PubMed PMID: 28739734.

86. Lagies S, Pichler R, Kaminski MM, Schlimpert M, Walz G, Lienkamp SS, Kammerer B. Metabolic characterization of directly reprogrammed renal tubular epithelial cells (iRECs). Scientific Reports. 2018;8(1):3878. doi: 10.1038/s41598018-22073-7.

87. Zhang P, Chen J-Q, Huang W-Q, Li W, Huang Y, Zhang Z-J, Xu F-G. Renal Medulla is More Sensitive to Cisplatin than Cortex Revealed by Untargeted Mass Spectrometry-Based Metabolomics in Rats. Scientific Reports. 2017;7(1):44804. doi: 10.1038/srep44804.

88. Boudonck KJ, Mitchell MW, Német L, Keresztes L, Nyska A, Shinar D, Rosenstock M. Discovery of Metabolomics Biomarkers for Early Detection of Nephrotoxicity. Toxicologic Pathology. 2009;37(3):280-92. doi: 10.1177/0192623309332992.

89. Portilla D, Li S, Nagothu KK, Megyesi J, Kaissling B, Schnackenberg L, Safirstein RL, Beger RD. Metabolomic study of cisplatin-induced nephrotoxicity. Kidney international. 2006;69(12):2194-204. doi: 10.1038/sj.ki.5000433. 
90. Zhang P, Li W, Chen J, Li R, Zhang Z, Huang Y, Xu F. Branched-Chain Amino Acids as Predictors for Individual Differences of Cisplatin Nephrotoxicity in Rats: A Pharmacometabonomics Study. Journal of Proteome Research. 2017;16(4):1753-62. doi: 10.1021/acs.jproteome.7b00014.

91. Wilmes A, Bielow C, Ranninger C, Bellwon P, Aschauer L, Limonciel A, Chassaigne H, Kristl T, Aiche S, Huber CG, Guillou C, Hewitt P, Leonard MO, Dekant W, Bois F, Jennings $P$. Mechanism of cisplatin proximal tubule toxicity revealed by integrating transcriptomics, proteomics, metabolomics and biokinetics. Toxicology in Vitro. 2015;30(1, Part A):117-27. doi: https://doi.org/10.1016/j.tiv.2014.10.006.

92. Li S, Nagothu K, Ranganathan G, Ali SM, Shank B, Gokden N, Ayyadevara S, Megyesi J, Olivecrona G, Chugh SS, Kersten S, Portilla D. Reduced kidney lipoprotein lipase and renal tubule triglyceride accumulation in cisplatin-mediated acute kidney injury. American Journal of Physiology-Renal Physiology. 2012;303(3):F437-F48. doi: 10.1152/ajprenal.00111.2012. PubMed PMID: 22622461.

93. Zhou R, Vander Heiden MG, Rudin CM. Genotoxic Exposure Is Associated with Alterations in Glucose Uptake and Metabolism. Cancer research. 2002;62(12):3515-20.

94. Choi YM, Kim HK, Shim W, Anwar MA, Kwon JW, Kwon HK, Kim HJ, Jeong H, Kim HM, Hwang D, Kim HS, Choi S. Mechanism of Cisplatin-Induced Cytotoxicity Is Correlated to Impaired Metabolism Due to Mitochondrial ROS Generation. PloS one. 2015;10(8):e0135083. doi: 10.1371/journal.pone.0135083. PubMed PMID: 26247588; PubMed Central PMCID: PMC4527592.

95. Ueda N, Kaushal GP, Shah SV. Apoptotic mechanisms in acute renal failure. The American journal of medicine. 2000;108(5):403-15. doi:

10.1016/s0002-9343(00)00311-9. PubMed PMID: 10759097.

96. Richter C, Gogvadze V, Laffranchi R, Schlapbach R, Schweizer M, Suter M, Walter P, Yaffee M. Oxidants in mitochondria: from physiology to diseases. Biochimica et biophysica acta. 1995;1271(1):67-74. doi: 10.1016/09254439(95)00012-s. PubMed PMID: 7599228.

97. Singh G. A possible cellular mechanism of cisplatin-induced nephrotoxicity. Toxicology. 1989;58(1):71-80. doi: 10.1016/0300-483x(89)901054. PubMed PMID: 2554535.

98. Gemba M, Fukuishi N. Amelioration by ascorbic acid of cisplatin-induced injury in cultured renal epithelial cells. Contributions to nephrology. 1991;95:13842. doi: 10.1159/000420651. PubMed PMID: 1807906.

99. Sadzuka Y, Shoji T, Takino Y. Effect of cisplatin on the activities of enzymes which protect against lipid peroxidation. Biochemical pharmacology. 1992;43(8):1872-5. doi: 10.1016/0006-2952(92)90725-x. PubMed PMID: 1575781.

100. Kruidering M, Van de Water B, de Heer E, Mulder GJ, Nagelkerke JF. Cisplatin-induced nephrotoxicity in porcine proximal tubular cells: mitochondrial dysfunction by inhibition of complexes I to IV of the respiratory chain. The Journal of pharmacology and experimental therapeutics. 1997;280(2):638-49. PubMed PMID: 9023274. 
101. Husain K, Morris C, Whitworth C, Trammell GL, Rybak LP, Somani SM. Protection by ebselen against cisplatin-induced nephrotoxicity: antioxidant system. Molecular and cellular biochemistry. 1998;178(1-2):127-33. doi: 10.1023/a:1006889427520. PubMed PMID: 9546591.

102. Huang Q, Dunn RT, 2nd, Jayadev S, DiSorbo O, Pack FD, Farr SB, Stoll $\mathrm{RE}$, Blanchard KT. Assessment of cisplatin-induced nephrotoxicity by microarray technology. Toxicological sciences : an official journal of the Society of Toxicology. 2001;63(2):196-207. doi: 10.1093/toxsci/63.2.196. PubMed PMID: 11568363.

103. Zunino F, Pratesi G, Micheloni A, Cavalletti E, Sala F, Tofanetti O. Protective effect of reduced glutathione against cisplatin-induced renal and systemic toxicity and its influence on the therapeutic activity of the antitumor drug. Chemico-biological interactions. 1989;70(1-2):89-101. doi: 10.1016/00092797(89)90065-3. PubMed PMID: 2736678.

104. Shiraishi F, Curtis LM, Truong L, Poss K, Visner GA, Madsen K, Nick HS, Agarwal A. Heme oxygenase-1 gene ablation or expression modulates cisplatininduced renal tubular apoptosis. American journal of physiology Renal physiology. 2000;278(5):F726-36. doi: 10.1152/ajprenal.2000.278.5.F726. PubMed PMID: 10807584.

105. Zsengellér ZK, Ellezian L, Brown D, Horváth B, Mukhopadhyay P, Kalyanaraman B, Parikh SM, Karumanchi SA, Stillman IE, Pacher P. Cisplatin nephrotoxicity involves mitochondrial injury with impaired tubular mitochondrial enzyme activity. J Histochem Cytochem. 2012;60(7):521-9. Epub 04/17. doi: 10.1369/0022155412446227. PubMed PMID: 22511597. 106. Nowak G. Protein kinase C-alpha and ERK1/2 mediate mitochondrial dysfunction, decreases in active $\mathrm{Na}+$ transport, and cisplatin-induced apoptosis in renal cells. The Journal of biological chemistry. 2002;277(45):43377-88. doi: 10.1074/jbc.M206373200. PubMed PMID: 12218054; PubMed Central PMCID: PMC1948818.

107. Park MS, De Leon M, Devarajan P. Cisplatin induces apoptosis in LLCPK1 cells via activation of mitochondrial pathways. Journal of the American Society of Nephrology : JASN. 2002;13(4):858-65. PubMed PMID: 11912244. 108. Vallon V. Tubular Transport in Acute Kidney Injury: Relevance for Diagnosis, Prognosis and Intervention. Nephron. 2016;134(3):160-6. doi: 10.1159/000446448.

109. Lan R, Geng H, Singha PK, Saikumar P, Bottinger EP, Weinberg JM, Venkatachalam MA. Mitochondrial Pathology and Glycolytic Shift during Proximal Tubule Atrophy after Ischemic AKI. Journal of the American Society of Nephrology. 2016;27(11):3356-67. doi: 10.1681/asn.2015020177.

110. Tao T, Yang X, Qin Q, Shi W, Wang Q, Yang Y, He J. NHERF1 Enhances Cisplatin Sensitivity in Human Cervical Cancer Cells. International journal of molecular sciences. 2017;18(1). doi: 10.3390/ijms18010005. PubMed PMID: 28085111; PubMed Central PMCID: PMC5297640.

111. Zsengeller ZK, Ellezian L, Brown D, Horvath B, Mukhopadhyay P, Kalyanaraman B, Parikh SM, Karumanchi SA, Stillman IE, Pacher P. Cisplatin nephrotoxicity involves mitochondrial injury with impaired tubular mitochondrial 
enzyme activity. J Histochem Cytochem. 2012;60(7):521-9. doi: 10.1369/0022155412446227. PubMed PMID: 22511597; PubMed Central PMCID: PMC3460350.

112. Hanigan MH, Lykissa ED, Townsend DM, Ou CN, Barrios R, Lieberman MW. Gamma-glutamyl transpeptidase-deficient mice are resistant to the nephrotoxic effects of cisplatin. The American journal of pathology. 2001;159(5):1889-94. doi: 10.1016/s0002-9440(10)63035-0. PubMed PMID: 11696449; PubMed Central PMCID: PMC1867073.

113. Hanigan MH, Gallagher BC, Taylor PT, Jr., Large MK. Inhibition of gamma-glutamyl transpeptidase activity by acivicin in vivo protects the kidney from cisplatin-induced toxicity. Cancer research. 1994;54(22):5925-9. PubMed PMID: 7954424.

114. Townsend DM, Marto JA, Deng M, Macdonald TJ, Hanigan MH. High pressure liquid chromatography and mass spectrometry characterization of the nephrotoxic biotransformation products of Cisplatin. Drug metabolism and disposition: the biological fate of chemicals. 2003;31(6):705-13. doi:

10.1124/dmd.31.6.705. PubMed PMID: 12756201; PubMed Central PMCID: PMC6522263.

115. Siddik ZH, Newell DR, Boxall FE, Harrap KR. The comparative pharmacokinetics of carboplatin and cisplatin in mice and rats. Biochemical pharmacology. 1987;36(12):1925-32. doi: 10.1016/0006-2952(87)90490-4. PubMed PMID: 3297068.

116. Perse M, Veceric-Haler Z. Cisplatin-Induced Rodent Model of Kidney Injury: Characteristics and Challenges. BioMed research international. 2018;2018:1462802. doi: 10.1155/2018/1462802. PubMed PMID: 30276200; PubMed Central PMCID: PMC6157122.

117. Jones DP, Liang Y. Measuring the poise of thiol/disulfide couples in vivo. Free radical biology \& medicine. 2009;47(10):1329-38. doi: 10.1016/j.freeradbiomed.2009.08.021. PubMed PMID: 19715755; PubMed Central PMCID: PMC2774737.

118. Watson WH, Burke TJ, Zelko IN, Torres-Gonzalez E, Ritzenthaler JD, Roman J. Differential Regulation of the Extracellular Cysteine/Cystine Redox State (EhCySS) by Lung Fibroblasts from Young and Old Mice. Oxid Med Cell Longev. 2016;2016:1561305. Epub 2016/09/20. doi: 10.1155/2016/1561305. PubMed PMID: 27642492; PubMed Central PMCID: PMC5014973.

119. Sadighi-Moghaddam B, Salek Farrokhi A, Namdar Ahmadabad H, Barati M, Moazzeni SM. Mesenchymal Stem Cell Therapy Prevents Abortion in CBA/J x DBA/2 Mating. Reproductive sciences. 2017:1933719117737848. doi:

10.1177/1933719117737848. PubMed PMID: 29187052.

120. Hannemann J, Baumann K. Cisplatin-induced lipid peroxidation and decrease of gluconeogenesis in rat kidney cortex: Different effects of antioxidants and radical scavengers. Toxicology. 1988;51(2):119-32. doi:

https://doi.org/10.1016/0300-483X(88)90143-6.

121. Timson DJ. Fructose 1,6-bisphosphatase: getting the message across. Biosci Rep. 2019;39(3):BSR20190124. doi: 10.1042/BSR20190124. PubMed PMID: 30804231. 
122. van Schaftingen E, Gerin I. The glucose-6-phosphatase system. Biochem J. 2002;362(Pt 3):513-32. doi: 10.1042/0264-6021:3620513. PubMed PMID: 11879177.

123. Valvona CJ, Fillmore HL, Nunn PB, Pilkington GJ. The Regulation and Function of Lactate Dehydrogenase A: Therapeutic Potential in Brain Tumor. Brain Pathology. 2016;26(1):3-17. doi: 10.1111/bpa.12299.

124. Gietl C. Malate dehydrogenase isoenzymes: Cellular locations and role in the flow of metabolites between the cytoplasm and cell organelles. Biochimica et Biophysica Acta (BBA) - Bioenergetics. 1992;1100(3):217-34. doi: https://doi.org/10.1016/0167-4838(92)90476-T.

125. Frenkel R. Regulation and Physiological Functions of Malic Enzymes. In: Horecker BL, Stadtman ER, editors. Current Topics in Cellular Regulation: Academic Press; 1975. p. 157-81.

126. Efferth T, Schwarzl SM, Smith J, Osieka R. Role of glucose-6-phosphate dehydrogenase for oxidative stress and apoptosis. Cell Death \& Differentiation. 2006;13(3):527-8. doi: 10.1038/sj.cdd.4401807.

127. Bonora M, Patergnani S, Rimessi A, De Marchi E, Suski JM, Bononi A, Giorgi C, Marchi S, Missiroli S, Poletti F, Wieckowski MR, Pinton P. ATP synthesis and storage. Purinergic Signal. 2012;8(3):343-57. Epub 04/12. doi: 10.1007/s11302-012-9305-8. PubMed PMID: 22528680.

128. Pfaller W, Thorwartl U, Nevinny-Stickel M, Krall M, Schober M, Joannidis M, Hobisch A. Clinical value of fructose 1,6 bisphosphatase in monitoring renal proximal tubular injury. Kidney international Supplement. 1994;47:S68-75.

PubMed PMID: 7532742.

129. Farooqui Z, Ahmed F, Rizwan S, Shahid F, Khan AA, Khan F. Protective effect of Nigella sativa oil on cisplatin induced nephrotoxicity and oxidative damage in rat kidney. Biomedicine \& Pharmacotherapy. 2017;85:7-15. doi: https://doi.org/10.1016/j.biopha.2016.11.110.

130. Yu W, Chen Y, Dubrulle J, Stossi F, Putluri V, Sreekumar A, Putluri N, Baluya D, Lai SY, Sandulache VC. Cisplatin generates oxidative stress which is accompanied by rapid shifts in central carbon metabolism. Scientific Reports. 2018;8(1):4306. doi: 10.1038/s41598-018-22640-y.

131. Phelps JS, Gandolfi AJ, Brendel K, Dorr RT. Cisplatin nephrotoxicity: in vitro studies with precision-cut rabbit renal cortical slices. Toxicology and applied pharmacology. 1987;90(3):501-12. doi: 10.1016/0041-008x(87)90142-6. PubMed PMID: 3310337.

132. Yilmaz HR, Iraz M, Sogut S, Ozyurt H, Yildirim Z, Akyol O, Gergerlioglu S. The effects of erdosteine on the activities of some metabolic enzymes during cisplatin-induced nephrotoxicity in rats. Pharmacological Research. 2004;50(3):287-90. doi: https://doi.org/10.1016/j.phrs.2004.03.003.

133. Per, \#x161, e M, Ve, \#x10d, eri, \#x107, -Haler, \#x17d, eljka. CisplatinInduced Rodent Model of Kidney Injury: Characteristics and Challenges. BioMed research international. 2018;2018:29. doi: 10.1155/2018/1462802.

134. Engelking LR. Chapter 27 - Metabolic Fates of Pyruvate. In: Engelking LR, editor. Textbook of Veterinary Physiological Chemistry (Third Edition). Boston: Academic Press; 2015. p. 169-73. 
135. Yao P, Sun H, Xu C, Chen T, Zou B, Jiang P, Du W. Evidence for a direct cross-talk between malic enzyme and the pentose phosphate pathway via structural interactions. The Journal of biological chemistry. 2017;292(41):1711320. doi: 10.1074/jbc.M117.810309. PubMed PMID: 28848047; PubMed Central PMCID: PMC5641861.

136. Zhang Z, Yang Z, Zhu B, Hu J, Liew CW, Zhang Y, Leopold JA, Handy $\mathrm{DE}$, Loscalzo J, Stanton RC. Increasing glucose 6-phosphate dehydrogenase activity restores redox balance in vascular endothelial cells exposed to high glucose. PloS one. 2012;7(11):e49128. doi: 10.1371/journal.pone.0049128. PubMed PMID: 23185302; PubMed Central PMCID: PMC3501497.

137. Kühlbrandt W. Structure and function of mitochondrial membrane protein complexes. BMC Biology. 2015;13(1):89. doi: 10.1186/s12915-015-0201-x.

138. Schapira AHV. Mitochondrial function and dysfunction. 2002. Amsterdam ;: Academic PressInternational review of neurobiology, 0074-7742 ; v. 53.

Available from: http://site.ebrary.com/id/10186474

http://search.ebscohost.com/login.aspx?direct=true\&scope=site \&db=nlebk\&db=nl abk\&AN=196143

http://www.myilibrary.com?id=105351

http://public.ebookcentral.proquest.com/choice/publicfullrecord.aspx?p=294268

http://www.sciencedirect.com/science/book/9780123668547

http://www.sciencedirect.com/science/bookseries/00747742/53

http://dproxy.library.dc-

uoit.ca/login?url=https://www.sciencedirect.com/science/bookseries/00747742/53

https://www.sciencedirect.com/science/bookseries/00747742/53

http://www.myilibrary.com?id=105351\&ref=toc

https://login.proxy.bib.uottawa.ca/login?url=http://ebookcentral.proquest.com/lib/o ttawa/detail.action?doclD $=294268$

https://ezproxy.aub.edu.lb/login?url=http://www.sciencedirect.com/science/books eries/00747742.

139. Oh G-S, Kim H-J, Shen A, Lee S-B, Yang S-H, Shim H, Cho E-Y, Kwon KB, Kwak TH, So H-S. New Therapeutic Concept of NAD Redox Balance for Cisplatin Nephrotoxicity. BioMed research international. 2016;2016:4048390-. Epub 01/05. doi: 10.1155/2016/4048390. PubMed PMID: 26881219.

140. Yang Y, Liu H, Liu F, Dong Z. Mitochondrial dysregulation and protection in cisplatin nephrotoxicity. Archives of Toxicology. 2014;88(6):1249-56. doi: 10.1007/s00204-014-1239-1.

141. Gordon JA, V. H. Gattone n. Mitochondrial alterations in cisplatin-induced acute renal failure. American Journal of Physiology-Renal Physiology.

1986;250(6):F991-F8. doi: 10.1152/ajprenal.1986.250.6.F991. PubMed PMID: 3717354.

142. Tanabe K, Tamura Y, Lanaspa MA, Miyazaki M, Suzuki N, Sato W, Maeshima Y, Schreiner GF, Villarreal FJ, Johnson RJ, Nakagawa T. Epicatechin limits renal injury by mitochondrial protection in cisplatin nephropathy. American Journal of Physiology-Renal Physiology. 2012;303(9):F1264-F74. doi:

10.1152/ajprenal.00227.2012. PubMed PMID: 22933302. 
143. Kharbangar A, Khynriam D, Prasad SB. Effect of cisplatin on mitochondrial protein, glutathione, and succinate dehydrogenase in Dalton lymphoma-bearing mice. Cell biology and toxicology. 2000;16(6):363-73. doi: 10.1023/a:1007648427024. PubMed PMID: 11254162.

144. Zhang L, Cooper AJ, Krasnikov BF, Xu H, Bubber P, Pinto JT, Gibson GE, Hanigan $\mathrm{MH}$. Cisplatin-induced toxicity is associated with platinum deposition in mouse kidney mitochondria in vivo and with selective inactivation of the alphaketoglutarate dehydrogenase complex in LLC-PK1 cells. Biochemistry. 2006;45(29):8959-71. doi: 10.1021/bi060027g. PubMed PMID: 16846239; PubMed Central PMCID: PMC4133109.

145. Chistiakov DA, Sobenin IA, Revin VV, Orekhov AN, Bobryshev YV. Mitochondrial aging and age-related dysfunction of mitochondria. BioMed research international. 2014;2014:238463-. Epub 04/10. doi:

10.1155/2014/238463. PubMed PMID: 24818134.

146. Brand MD, Nicholls DG. Assessing mitochondrial dysfunction in cells. Biochem J. 2011;435(2):297-312. doi: 10.1042/BJ20110162. PubMed PMID: 21726199.

147. Das KC, Muniyappa H. Age-dependent mitochondrial energy dynamics in the mice heart: role of superoxide dismutase-2. Exp Gerontol. 2013;48(9):94759. Epub 06/24. doi: 10.1016/j.exger.2013.06.002. PubMed PMID: 23806974. 148. Shenolikar S, Voltz JW, Minkoff CM, Wade JB, Weinman EJ. Targeted disruption of the mouse NHERF-1 gene promotes internalization of proximal tubule sodium-phosphate cotransporter type Ila and renal phosphate wasting. Proceedings of the National Academy of Sciences. 2002;99(17):11470-5. doi: 10.1073/pnas.162232699.

149. Pabla N, Dong Z. Cisplatin nephrotoxicity: Mechanisms and renoprotective strategies. Kidney international. 2008;73(9):994-1007. doi:

https://doi.org/10.1038/sj.ki.5002786.

150. Ciarimboli G. Membrane transporters as mediators of cisplatin sideeffects. Anticancer research. 2014;34(1):547-50. PubMed PMID: 24403515. 151. Fliedl L, Wieser M, Manhart G, Gerstl MP, Khan A, Grillari J, GrillariVoglauer R. Controversial role of gamma-glutamyl transferase activity in cisplatin nephrotoxicity. Altex. 2014;31(3):269-78. doi: 10.14573/altex.1311152. PubMed PMID: 24664430.

152. Lieberthal W, Triaca V, Levine J. Mechanisms of death induced by cisplatin in proximal tubular epithelial cells: apoptosis vs. necrosis. American Journal of Physiology-Renal Physiology. 1996;270(4):F700-F8. doi: 10.1152/ajprenal.1996.270.4.F700. PubMed PMID: 8967349. 153. Hwa Lee R, Mi Song J, Young Park M, Kyung Kang S, Keun Kim Y, Sup Jung J. Cisplatin-induced apoptosis by translocation of endogenous Bax in mouse collecting duct cells11Abbreviations: ROS, reactive oxygen species; SAPK/JNK, stress-activated protein kinase/c-Jun NH2-terminal kinase; RT-PCR, reverse transcription-polymerase chain reaction; ECL, enhanced

chemiluminescence; LDH, lactic dehydrogenase; DPPD, diphenyl-p-phenylenediamine; DFO, deferoxamine; DMTU, dimethylthiourea; and BHA, butylated 
hydroxyanisole. Biochemical pharmacology. 2001;62(8):1013-23. doi: https://doi.org/10.1016/S0006-2952(01)00748-1.

154. Saleena Ummer B MA, BM vadhiraha, DH Fernandes, R prabhu, Nalini K. Evaluation of urinary tubular enzymes for the detection of early kidney injury due to cisplatin chemotherapy. Int J Biol Med Res. 2012;3(3):2241-6.

155. Jia Z, Wang N, Aoyagi T, Wang H, Liu H, Yang T. Amelioration of cisplatin nephrotoxicity by genetic or pharmacologic blockade of prostaglandin synthesis. Kidney international. 2011;79(1):77-88. doi: 10.1038/ki.2010.331. PubMed PMID: 20844471.

156. Ognjanović BI, Djordjević NZ, Matić MM, Obradović JM, Mladenović JM, Stajn AŠ, Saičić ZS. Lipid peroxidative damage on Cisplatin exposure and alterations in antioxidant defense system in rat kidneys: a possible protective effect of selenium. Int J Mol Sci. 2012;13(2):1790-803. Epub 02/08. doi: 10.3390/ijms13021790. PubMed PMID: 22408424.

157. González R, Romay C, Borrego A, Hernández F, Merino N, Zamora Z, Rojas E. Lipid Peroxides and Antioxidant Enzymes in Cisplatin-Induced Chronic Nephrotoxicity in Rats. Mediators of Inflammation. 2005;2005(3). doi: 10.1155/mi.2005.139.

158. Tokunaga J, Kobayashi M, Kitagawa A, Nakamura C, Arimori K, Nakano $M$. Protective effects of betamipron on renal toxicity during repeated cisplatin administration in rats and protective mechanism. Renal failure. 1998;20(1):27-38. doi: 10.3109/08860229809045087. PubMed PMID: 9509558.

159. Hajian S, Rafieian-Kopaei M, Nasri H. Renoprotective effects of antioxidants against cisplatin nephrotoxicity. J Nephropharmacol. 2014;3(2):3942. PubMed PMID: 28197460.

160. Verma PK, Raina R, Sultana M, Singh M, Kumar P. Total antioxidant and oxidant status of plasma and renal tissue of cisplatin-induced nephrotoxic rats: protection by floral extracts of Calendula officinalis Linn. Renal failure. 2016;38(1):142-50. doi: 10.3109/0886022X.2015.1103585. PubMed PMID: 26513373.

161. Yu X, Long YC. Crosstalk between cystine and glutathione is critical for the regulation of amino acid signaling pathways and ferroptosis. Sci Rep. 2016;6:30033-. doi: 10.1038/srep30033. PubMed PMID: 27425006.

162. Paolicchi A, Sotiropuolou M, Perego P, Daubeuf S, Visvikis A, Lorenzini E, Franzini M, Romiti N, Chieli E, Leone R, Apostoli P, Colangelo D, Zunino F, Pompella A. Y-Glutamyl transpeptidase catalyses the extracellular detoxification of cisplatin in a human cell line derived from the proximal convoluted tubule of the kidney. European Journal of Cancer. 2003;39(7):996-1003. doi: https://doi.org/10.1016/S0959-8049(03)00067-4.

163. Dobyan DC, Levi J, Jacobs C, Kosek J, Weiner MW. Mechanism of cisplatinum nephrotoxicity: II. Morphologic observations. The Journal of pharmacology and experimental therapeutics. 1980;213(3):551-6. PubMed PMID: 7193726.

164. Sanz N, Díez-Fernández C, Valverde AM, Lorenzo M, Benito M, Cascales M. Malic enzyme and glucose 6-phosphate dehydrogenase gene expression increases in rat liver cirrhogenesis. British journal of cancer. 1997;75(4):487-92. 
165. González R, Romay C, Borrego A, Hernández F, Merino N, Zamora Z, Rojas E. Lipid peroxides and antioxidant enzymes in cisplatin-induced chronic nephrotoxicity in rats. Mediators Inflamm. 2005;2005(3):139-43. doi:

10.1155/MI.2005.139. PubMed PMID: 16106099.

166. Larochelle M, Drouin S, Robert F, Turcotte B. Oxidative stress-activated zinc cluster protein Stb5 has dual activator/repressor functions required for pentose phosphate pathway regulation and NADPH production. Molecular and cellular biology. 2006;26(17):6690-701. doi: 10.1128/MCB.02450-05. PubMed PMID: 16914749; PubMed Central PMCID: PMC1592823.

167. Nguyen TT, Quan X, Hwang K-H, Xu S, Das R, Choi S-K, Wiederkehr A, Wollheim CB, Cha S-K, Park K-S. Mitochondrial oxidative stress mediates highphosphate-induced secretory defects and apoptosis in insulin-secreting cells. American Journal of Physiology-Endocrinology and Metabolism. 2015;308(11):E933-E41. doi: 10.1152/ajpendo.00009.2015. PubMed PMID: 25852001.

168. Oda M, Koyanagi S, Tsurudome Y, Kanemitsu T, Matsunaga N, Ohdo S. Renal circadian clock regulates the dosing-time dependency of cisplatin-induced nephrotoxicity in mice. Molecular pharmacology. 2014;85(5):715-22. doi:

10.1124/mol.113.089805. PubMed PMID: 24567546.

169. Oda M, Koyanagi S, Tsurudome Y, Kanemitsu T, Matsunaga N, Ohdo S. Renal Circadian Clock Regulates the Dosing-Time Dependency of CisplatinInduced Nephrotoxicity in Mice. Molecular Pharmacology. 2014;85(5):715-22. doi: $10.1124 / \mathrm{mol} .113 .089805$.

170. Cooper AJL, Krasnikov BF, Niatsetskaya ZV, Pinto JT, Callery PS, Villar MT, Artigues A, Bruschi SA. Cysteine S-conjugate $\beta$-lyases: important roles in the metabolism of naturally occurring sulfur and selenium-containing compounds, xenobiotics and anticancer agents. Amino Acids. 2011;41(1):7-27. Epub 03/22. doi: 10.1007/s00726-010-0552-0. PubMed PMID: 20306345.

171. Dunham-Snary KJ, Sandel MW, Westbrook DG, Ballinger SW. A method for assessing mitochondrial bioenergetics in whole white adipose tissues. Redox Biol. 2014;2:656-60. doi: 10.1016/j.redox.2014.04.005. PubMed PMID: 24936439.

172. Neville KE, Bosse TL, Klekos M, Mills JF, Weicksel SE, Waters JS, Tipping M. A novel ex vivo method for measuring whole brain metabolism in model systems. Journal of Neuroscience Methods. 2018;296:32-43. doi: https://doi.org/10.1016/j.jneumeth.2017.12.020.

173. Horan MP, Pichaud N, Ballard JWO. Review: Quantifying Mitochondrial Dysfunction in Complex Diseases of Aging. The Journals of Gerontology: Series A. 2012;67(10):1022-35. doi: 10.1093/gerona/glr263.

174. Lash LH. Methods for measuring cysteine S-conjugate beta-lyase activity. Current protocols in toxicology. 2007; Chapter 6:Unit6 13. doi:

10.1002/0471140856.tx0613s34. PubMed PMID: 23045149.

175. Sharp CN, Doll MA, Dupre TV, Shah PP, Subathra M, Siow D, Arteel GE, Megyesi J, Beverly LJ, Siskind LJ. Repeated administration of low-dose cisplatin in mice induces fibrosis. American Journal of Physiology-Renal Physiology. 
2016;310(6):F560-F8. doi: 10.1152/ajprenal.00512.2015. PubMed PMID: 26739893. 


\section{ABBREVIATIONS}

\begin{tabular}{|c|c|}
\hline ACEI & Angiotensin converting enzyme inhibitors \\
\hline AGN & Acute glomerulonephritis \\
\hline AIN & Acute interstitial nephritis \\
\hline AKI & Acute kidney injury \\
\hline ANOVA & Analysis of variance \\
\hline AP & Aminopeptidase \\
\hline ARB & Angiotensin receptor blockers \\
\hline ATN & Acute tubular necrosis \\
\hline ATPase & Adenosine triphosphatase \\
\hline BBM & Brush border membrane \\
\hline BCA & Bicinchoninic acid \\
\hline BSA & Bovine serum albumin \\
\hline BUN & Blood urea nitrogen \\
\hline $\mathrm{CCBL}$ & Cysteine-S-conjugate beta lyase \\
\hline CCBL1 & Alternative name for glutamate transaminase $k$ \\
\hline CKD & Chronic kidney disease \\
\hline CRAC & Cholesterol binding domain \\
\hline Ctr1 & Copper transporter 1 \\
\hline CySSG & Cysteine-glutathione disulfide \\
\hline Cys & Cysteine \\
\hline Cyss & Cystine \\
\hline CXCR2 & C-X-C motif chemokine receptor 2 \\
\hline E. coli & Escherichia coli \\
\hline EGFR & Epidermal growth factor receptor \\
\hline EGTA & Ethylene glycol tetraacetic acid \\
\hline
\end{tabular}




\begin{tabular}{|c|c|}
\hline ELISA & Enzyme-linked immunosorbent assay \\
\hline EM & Electron microscopy \\
\hline EMT & Epithelial-mesenchymal-transition \\
\hline ERM & Ezrin-radixin-moesin \\
\hline ETC & Electron transport chain \\
\hline FBPase & Fructose-1,6-bisphosphatase \\
\hline FDA & Food and drug administration \\
\hline GAPDH & Glyceraldehyde 3-phosphate dehydrogenase \\
\hline GFR & Glomerular filtration rate \\
\hline GGT & Y glutamyl transpeptidase or transferase \\
\hline GI & Gastrointestinal \\
\hline GPCRs & G-protein-coupled-receptors \\
\hline GSH & Glutathione \\
\hline GSSG & Glutathione disulfide \\
\hline GTK & Glutamate transaminase $\mathrm{k}$ \\
\hline G6Pase & Glucose-6-phosphatase \\
\hline G6PD & Glucose-6-phosphate dehydrogenase \\
\hline HEPES & 4-(2-hydroxylethyl)-1-piperazineethanesulfonic acid \\
\hline$H \& E$ & Hematoxylin and eosin \\
\hline HPLC & High-performance-liquid-chromatography \\
\hline IACUC & Institutional animal care and use committee \\
\hline ICAM-1 & Intracellular adhesion molecule 1 \\
\hline ICU & Intensive care unit \\
\hline $\mathrm{IHC}$ & Immunohistochemistry \\
\hline IL8 & Interleukin 8 \\
\hline IP & Intraperitoneal \\
\hline JM & Juxtamedullary \\
\hline $\mathrm{KO}$ & Knock out \\
\hline LC-MS & Liquid chromatography-mass spectrometry \\
\hline
\end{tabular}




\begin{tabular}{|c|c|}
\hline $\mathrm{LDH}$ & Lactate dehydrogenase \\
\hline MATE1 & Multidrug and toxin extrusion 1 \\
\hline MDA & Malondialdehyde \\
\hline MDCK & Madrin-darby-canine-kidney \\
\hline $\mathrm{MDH}$ & Malate dehydrogenase \\
\hline MOPS & 3-(N-morpholino)propanesulonic acid \\
\hline $\mathrm{mOsm} / \mathrm{kg}$ & Milliosmoles per kilogram \\
\hline ME & Malic enzyme \\
\hline NF-KB & $\begin{array}{l}\text { Nuclear factor kappa-light chain enhancer of activated B } \\
\text { cells }\end{array}$ \\
\hline NGAL & Neutrophil gelatinase-associated lipocalin \\
\hline NHERF1 & $\mathrm{Na} / \mathrm{H}$ exchange regulatory factor 1 \\
\hline NHE3 & Sodium hydrogen antiporter 3 \\
\hline Nox1 & NADPD oxidase \\
\hline Npt2a & Sodium phosphate co-transporter type-2 \\
\hline NSAID & Nonsteroidal anti-inflammatory drug \\
\hline OCR & Oxygen consumption rate \\
\hline ОСТ2 & Organic cation transporter 2 \\
\hline OK & Opossum kidney \\
\hline PAS & Periodic acid-schiff \\
\hline PBS & Phosphate buffered saline \\
\hline PDZ & $\begin{array}{l}\text { Post-synaptic density protein 95/drosophila discs } \\
\text { large/zonulaocculens-1 }\end{array}$ \\
\hline $\mathrm{Pt}$ & Platinum \\
\hline PTH & Parathyroid hormone \\
\hline $\mathrm{RCR}$ & Respiratory control ratio \\
\hline ROCK & Rho-associated coiled-coil containing protein kinase \\
\hline ROS & Reactive oxygen species \\
\hline SEM & Standard error means \\
\hline SNP & Single-nucleotide polymorphism \\
\hline Stat3 & Single transducer and activator of transcription 3 \\
\hline
\end{tabular}


TBARS Thiobarbituric acid reactive substances

TCA Trichloroacetic acid

TGF- $\beta 1 \quad$ Transforming growth factor beta 1

TTBS Tris-buffered saline with $0.5 \%$ Tween 20

WT Wild type

VEGF Vascular endothelial growth factor

4-HNE 4-hydroxylnonenal 


\section{PUBLISHER'S NOTICE OF APPROVAL}

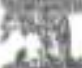

Tixitis

Oear Adrimen listwuseritile

Thank you for your enat

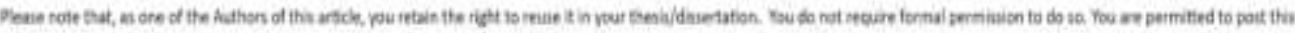

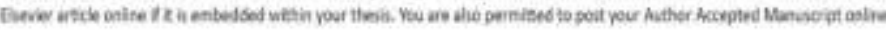

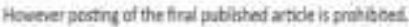

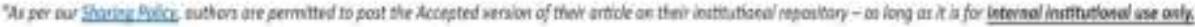

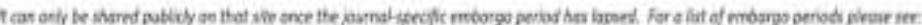

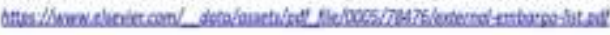

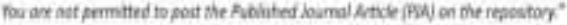

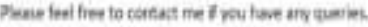

Kendugardi.

Kaveil

Pentituosis kejodes 


\title{
CURRICULUM VITAE
}

\author{
Adrienne M. Bushau-Sprinkle \\ University of Louisville School of Medicine \\ Department of Pharmacology and Toxicology \\ Louisville, KY 40292
}

\section{EDUCATION}

Ph.D. in Pharmacology and Toxicology (expected graduation May 2020)

University of Louisville, Louisville, KY

M.S. in Pharmacology and Toxicology

2018

University of Louisville, Louisville, KY

B.S. in Chemistry with a concentration in Biochemistry

2015

Minor in Biology

University of Louisville, Louisville, KY

\section{RESEARCH EXPERIENCE}

\section{Doctoral Candidate-Graduate Research Fellow}

2015-present

Department of Pharmacology \& Toxicology, University of Louisville, Louisville, KY

Mentor: Eleanor D. Lederer, M.D.

- Project Title: Potential biomarkers of cisplatin-induced acute kidney injury human subject study.

- Project Title: Metabolic differences of NHERF1 knock out mice and sensitivity to cisplatin nephrotoxicity. 
- Project Title: The projective role of the sodium hydrogen exchange regulatory factor 1 and cisplatin-induced acute kidney injury.

\section{Undergraduate Student Research Assistant}

2014-2015

Department of Pharmacology \& Toxicology, University of Louisville, Louisville, KY

Mentor: Juliane Beier, Ph.D.

- Project Title: The effects of vinyl chloride on endoplasmic reticulum stress and hepatic injury.

Undergraduate Student Research Assistant

2012-2013

Department of Biochemistry and Molecular Genetics, University of Louisville, Louisville, $\mathrm{KY}$

Mentor: Chuan Hu, Ph.D.

- Project Title: Chemopreventative food components and the dynamin inhibitor, dynasore, as a treatment for pancreatic cancer.

High School Student Summer Fellow

2008

Department of Biochemistry \& Molecular Genetics, University of Louisville, Louisville, KY

Mentor: James Wittliff, Ph.D.

- Project: Comparing the effects of estrogen mimics and breast carcinoma.

\section{AREAS OF RESEARCH EXPERTISE}

- Cisplatin nephrotoxicity

- Acute kidney injury

- In vivo models of kidney injury

- Urine exosome isolation techniques

- Primary proximal tubule cell

- Mitochondrial isolation techniques

- Quantitative PCR

- Tissue staining

- Renal pathology culture 


\section{SKILLS}

- Proficient in Microsoft Office including Word, Excel, and PowerPoint

- Experience with graphing and statistical analysis using GraphPad Prism and SigmaPlot

- Familiar with reference manager Endnote

- Experience with IRB submissions (protocol, informed consent form, and amendments)

- Training in laboratory biorepository maintenance

\section{ACADEMIC \& PROFESSIONAL HONORS}

\section{Traineeships}

- NIH R25 Cancer Education Program Trainee, 2013

- NIH R25 Cancer Education Program Trainee, 2012

- Institute for Molecular Diversity and Drug Design Program, 2012

- Summer Research Opportunities Program Trainee, 2011

Fellowships

- Integrated Program in Biomedical Sciences Fellowship, 2015-2017

\section{Awards}

- Finalist for the 2019 Research Louisville Pre-doctoral Graduate Student Award at the University of Louisville's Research Louisville Conference in Louisville, $\mathrm{KY}$

- Finalist for the 2019 American Physiological Society (APS) Renal Section Pre-doctoral Award at Experimental Biology Conference in Orlando, FL

- Graduate Student Council Travel Award, 2019

- Finalist for the 2017 Cell and Molecular Physiological Society (CaMPS) Robert Gunn Student Travel Award at Experimental Biology Conference in Chicago, IL

- Class Representative, Department of Pharmacology and Toxicology, University of Louisville, Louisville, KY (2015-2018)

- $\mathrm{NCl}$ Outstanding Cancer Research Presentation Award, 2012

\section{Professional Societies}

- American Society of Nephrology, 2016-present

- American Society for Pharmacology and Experimental Therapeutics, 2016-present

- University of Louisville Kidney Disease Program, 2015-present

- University of Louisville Science Policy and Outreach Group, 2015-present

\section{Service to the Profession}

07/2018 Collected clinical data for Superfund "Project Greenheart"

07/2018 Led roundtable discussion for NCI R25 Cancer Education Program

Fellows 
05/2018 Interviewed Ph.D. applicants for the Pharmacology and Toxicology

Department

01/2018 Volunteer science fair project judge at the National United States Pony Clubs Conference

05/2017 Interviewed Ph.D. applicants for the Pharmacology and Toxicology Department

07/2016 Led roundtable discussion for NCI R25 Cancer Education Program Fellows

05/2016 Interviewed Ph.D. applicants for the Pharmacology and Toxicology Department

\section{SELECTED ABSTRACTS \& POSTER PRESENTATIONS}

\section{Local/Regional}

1. Bushau-Sprinkle A., Watson W., Zheng Y., Gagnon K., Kitterman K., Barati M., Siskind L., Brier M., Lederer E. (2019) Mechanistic insight for increased susceptibility to cisplatin nephrotoxicity with NHERF1 loss. Research!Louisville Abs. \#GRD-18, September 10-13, 2019, Louisville, $\mathrm{KY}$.

2. Bushau-Sprinkle A., Conklin C., Barati M., Dupre T., Gagnon K., Siskind L., Doll M., Rane M., Clark B., Merchant M., Klinge C., Brier M., Coventry S., Lederer E. (2018) NHERF1 loss results in metabolic stress and increased susceptibility to cisplatin-induced acute kidney injury.

Research!Louisville Abs. \#GRD-3, October 10-12, 2018, Louisville, KY.

3. Bushau-Sprinkle A. Dupre T., Siskind L., Sherwood A., Conklin C., Barati M., Gagnon K., Khundmiri S., and Lederer E. (2016) The protective role of NHERF1 in cisplatin-induced acute kidney injury. Research!Louisville Abs. \#GRM-3, October 11-13, 2016, Louisville, KY.

4. Sherwood A., Conklin C., Barati M., Gagnon K., Bushau-Sprinkle A., Merchant M., Lederer E., Khundmiri S. (2016) Mass spectrometry and cellular bioenergetics analysis reveals altered mitochondrial function in the kidneys of $\mathrm{Na}-\mathrm{H}$ exchanger regulatory factor isoform 1 (NHERF1) deficient mice. Research!Louisville Abs. \#PRF-17, October 11-13, 2016, Louisville, KY.

5. Sheehan R., Korte E., Bushau A., Powell D., Merchant M. (2015) ABIN1 ubiquitin binding regulates the proteome of exosomes derived from podocytes. Research!Louisville. Louisville, KY.

6. Bushau A., Anders L., Douglas A., Joshi-Barve S., Poole L., Massey V., Falkner K., Cave M., McClain C., and Beier J. (2014) Mechanistic insight into vinyl chloride-induced liver injury. Ohio Valley Society of Toxicology (OVSOT) Annual Meeting, Dayton, $\mathrm{OH}$. 
7. Bushau A., Andres S., and Wittliff J. (2013) Gene expression in breast carcinomas from patients with ethnical differences. Research!Louisville, Louisville, KY (National Cancer Institute Outstanding Cancer Research Award)

8. Bushau A., and Hu C. The effects of chemo preventative food components and dynasore (dynamin inhibitor) on $\beta-1$ integrin trafficking and cell proliferation in pancreatic cancer cells. (2011) The University of Louisville's Summer Research Opportunity Poster Session, Louisville, KY.

\section{National/International}

1. Bushau-Sprinkle A., Conklin C., Barati M., Dupre T., Gagnon K., Siskind L., Doll M., Rane M., Clark B., Merchant M., Klinge C., Brier M., Coventry S., Lederer E. (2019) NHERF1 loss results in metabolic stress and increased susceptibility to cisplatin-induced acute kidney injury. (2019) Experimental Biology Annual Meeting Abs. \#3438, April 6-9, 2019, Orlando, FL.

2. Bushau-Sprinkle A., Conklin C., Barati M., Brier M., Coventry S., Dupre T., Siskind L., Rane M., and Lederer E. (2017) NHERF1 deficiency increases susceptibility to cisplatin-induced acute kidney injury. American Society of Nephrology Kidney Week Abs. \#TH-PO306, November 1-5, 2017, New Orleans, LA.

3. Bushau-Sprinkle A., Sherwood A., Conklin C., Barati M., Dupre T., Siskind L., Gagnon K., Khundmiri S., and Lederer E. (2017) The protective role of NHERF1 in cisplatin-induced acute kidney injury. Experimental Biology Annual Meeting Abs. \#7605, April 22-26, 2017, Chicago, IL.

4. Anders L., Yeo H., Kaelin B., Bushau A., Lang A., Arteel G., McClain C. and Beier J. (2016) Role of dietary fatty acids in liver injury caused by vinyl chloride metabolites in mice. Hepatology 64:769A. (Presidential Poster of Distinction).

5. Sherwood A., Khundmiri S., Conklin C., Siskind L., Bushau A., Dupre T., Barati M., Merchant M., Lederer E. (2016) Increased susceptibility to cisplatin-induced acute kidney injury in the kidneys of $\mathrm{Na}-\mathrm{H}$ exchanger regulatory factor isoform 1 deficient mice. Experimental Biology Annual Meeting, Abs. \#B170, April 2-6, 2016, San Diego, CA.

6. Bushau A., Anders L., Douglas A., Joshi-Barve S., Poole L., Massey V., Falkner K., Cave M., McClain C., and Beier J. Mechanistic insight into vinyl chloride-induced liver injury. Society of Toxicology Annual Meeting, Abs. \#125, March 22-26, 2015, San Diego, CA.

7. Anders L., Douglas A. Bushau A., Falkner K., Arteel G., Cave M., McClain C. and Beier J. (2014) Exposure to vinyl chloride metabolites exacerbates liver injury caused by high fat diet in mice. Hepatology 60(1):719A. (Presidential Poster Distinction) 


\section{PUBLICATIONS}

1. Bushau-Sprinkle A. and Lederer E. New roles of the $\mathrm{Na}^{+} / \mathrm{H}^{+}$exchange regulatory factor 1 scaffolding protein: a mini review. AJP Renal. 2019 (under review)

2. Bushau-Sprinkle A. and Andres S.A. Effects of inflatable air vests and prevention in equestrian injuries. (in progress)

3. Bushau-Sprinkle A., Barati M., Conklin C., Dupre, T., Gagnon K., Khundmiri, S., Clark B., Siskind L., Doll M., Rane M., Brier M., Coventry S., Lederer E. Loss of the $\mathrm{Na}^{+} / \mathrm{H}^{+}$exchange regulatory factor 1 increases susceptibility to cisplatin-induced AKI. Am. J. Pathol. 2019 June; 159(6):1190-1200.

4. Andres S., Bushau-Sprinkle A., Brier M., and Seger Y. Effects of body protection vests and experience levels in prevention of equestrian injuries. BMJ Open Sport \& Exercise Medicine. 2018; 4:e000426. Doi: 10.1136/

5. Anders L., Lang A., Anwar A., Douglas A., Bushau A., Falkner K., Hill B., Warner N., Arteel G., Cave M., McClain C., and Beier J. Vinyl chloride metabolites potentiate inflammatory liver injury caused by LPS in mice. Toxicol. Sci. 2016 June 8;151(2):312-23.

6. Anders L., Yeo H., Kaelin B., Lang A., Bushau, A., Douglas A., Cave M., Arteel G., McClain C., and Beier J. Role of dietary fatty acids in liver injury caused by vinyl chloride metabolites in mice. Toxicol Appl Pharmacol. 2016 Sep 28.

\section{PRESENTATIONS}

1. Oral Presentation, 09/19, NHERF1: more than an anchor. University of Louisville, Pharmacology and Toxicology Departmental Seminar, Louisville, KY.

2. Poster Presentation Competition, 09/19, Mechanistic insight for increased susceptibility to cisplatin nephrotoxicity with NHERF1 loss ( ${ }^{\text {rd }}$ place poster). University of Louisville's Research!Louisville, Louisville, KY.

3. Poster Presentation Competition, 04/19, NHERF1 loss results in metabolic stress and increased susceptibility to cisplatin-induced acute kidney injury ( $3^{\text {rd }}$ place poster). American Physiological Society Posters with Professors Event at the Experimental Biology Annual Meeting, Orlando, FL. 
4. Poster, 04/19, NHERF1 loss results in metabolic stress and increased susceptibility to cisplatin-induced acute kidney injury. Experimental Biology Annual Meeting, Orlando, FL,

5. Poster, 10/18, NHERF1 loss results in metabolic stress and increased susceptibility to cisplatin-induced acute kidney injury. University of Louisville's Research!Louisville, Louisville, KY.

6. Master's Defense and Ph.D. Proposal, $12 / 17$, Loss of the $\mathrm{Na}^{+} / \mathrm{H}^{+}$ exchange regulatory factor 1 results in increased susceptibility to cisplatininduced acute kidney injury. University of Louisville, Pharmacology \& Toxicology Departmental Seminar, Louisville, KY.

7. Poster, 11/17, NHERF1 deficiency increases susceptibility to cisplatininduced acute kidney injury. American Society of Nephrology Kidney Week, New Orleans, LA.

8. Oral Presentation, 04/17, The protective role of NHERF1 in cisplatininduced acute kidney injury. Experimental Biology Annual Meeting, Session: New Insights into Renal Transport: Application of Genetic Models (Featured Topic), Chicago, IL.

9. Poster, 04/17, The protective role of NHERF1 in cisplatin-induced acute kidney injury. Experimental Biology Annual Meeting, Chicago, IL.

10. Poster Presentation Competition, 04/17, The protective role of NHERF1 in cisplatin-induced acute kidney injury. Cell and Molecular Physiological Society session at the Experimental Biology Annual Meeting, Chicago, IL.

11. Poster, 10/16, The protective role of NHERF1 in cisplatin-induced acute kidney injury. University of Louisville's Research!Louisville, Louisville, KY.

12. Research Seminar, 04/16, The protective role of NHERF1 in cisplatininduced acute kidney injury. University of Louisville, Pharmacology \& Toxicology Department Seminar, Louisville, KY.

13. Poster, 03/15, Mechanistic insight into vinyl chloride-induced liver injury. Society of Toxicology Annual Meeting, San Diego, CA.

14. Poster, 10/14 Mechanistic insight into vinyl chloride-induced liver injury. Ohio Valley Society of Toxicology (OVSOT) Annual Meeting, Dayton, $\mathrm{OH}$.

15. Poster, 04/13, Gene expression in breast carcinomas from patients with ethnical differences (National Cancer Institute Outstanding Cancer Research Presentation). University of Louisville, Research!Louisville, Louisville, KY.

16. Poster, 08/11, The effects of chemo preventative food components and 
dynasore (dynamin inhibitor) on $\beta-1$ integrin trafficking and cell proliferation in pancreatic cancer cells. The University of Louisville's Summer Research Opportunity Poster Session, Louisville, KY. 